\title{
Factors Influencing Huntingtin Aggregation at Surfaces: Implications for Huntington's Disease
}

\author{
Sharon E. Groover \\ West Virginia University, sgroover@mix.wvu.edu
}

Follow this and additional works at: https://researchrepository.wvu.edu/etd

Part of the Biophysics Commons, Molecular and Cellular Neuroscience Commons, Other

Biochemistry, Biophysics, and Structural Biology Commons, and the Physical Chemistry Commons

\section{Recommended Citation}

Groover, Sharon E., "Factors Influencing Huntingtin Aggregation at Surfaces: Implications for Huntington's Disease" (2020). Graduate Theses, Dissertations, and Problem Reports. 7937.

https://researchrepository.wvu.edu/etd/7937

This Dissertation is protected by copyright and/or related rights. It has been brought to you by the The Research Repository @ WVU with permission from the rights-holder(s). You are free to use this Dissertation in any way that is permitted by the copyright and related rights legislation that applies to your use. For other uses you must obtain permission from the rights-holder(s) directly, unless additional rights are indicated by a Creative Commons license in the record and/ or on the work itself. This Dissertation has been accepted for inclusion in WVU Graduate Theses, Dissertations, and Problem Reports collection by an authorized administrator of The Research Repository @ WVU.

For more information, please contact researchrepository@mail.wvu.edu. 


\title{
Factors Influencing Huntingtin Aggregation at Surfaces: Implications for Huntington's Disease
}

\section{Sharon E. Groover}

\author{
Dissertation submitted to the \\ Eberly College of Arts and Science \\ at West Virginia University \\ Morgantown, WV 26505
}

in partial fulfillment of the requirements for the degree of

\author{
Doctor of Philosophy \\ in \\ Chemistry
}

Justin Legleiter, PhD Advisor and Committee Chair John Blakely Mertz, PhD

Visvanathan Ramamurthy, $\mathrm{PhD}$

Kenneth Showalter, PhD

Stephen Valentine, PhD

C. Eugene Bennett Department of Chemistry

Morgantown, WV

2020

Keywords: Huntington's Disease, amyloid formation, phosphorylation, macromolecular crowders, atomic force microscopy, lipids, peptide 


\title{
Factors Influencing Huntingtin Aggregation at Surfaces: Implications for Huntington's Disease
}

\author{
Abstract \\ Sharon E. Groover
}

Huntington's Disease (HD) is a genetic, neurodegenerative disease characterized by an abnormal polyglutamine (polyQ) expansion in the first exon of the huntingtin protein (htt). The polyQ domain facilitates aggregation and initiates the formation of a diverse collection of aggregate species, including fibrils, oligomers and annular aggregates. The first 17 amino acids of htt (Nt17) directly flank the polyQ domain and is a key factor in htt's association to membranous structures. In addition to Nt17 being an amphipathic $\alpha$ helix, it also promotes aggregation through self-association and contains numerous posttranslational modifications (PTMs) that can modulate toxicity and subcellular localization. For in depth understanding of these mechanisms, particularly in the presence of lipid membrane surfaces, the PTM phosphorylation and macromolecular crowders found in subcellular environments were explored. Through the application of phosphomimetic mutations of htt to a variety of lipid systems, lipid-specific impacts of electrostatic interactions involved in htt/lipid interactions were elucidated. Cytosolic conditions mimicked through the addition of macromolecular crowders and htt were evaluated at both solid/liquid and membrane/liquid interfaces, with each crowder having a distinct effect on htt aggregation. The results presented here aid in the understanding of the multi-faceted nature of htt aggregation in the presence of cellular and subcellular surfaces 


\section{Acknowledgements}

Completing the works presented here would not have been possible without the support, guidance, and assistance of many special and extraordinary people. I would like to express my genuine thanks and deepest appreciation to those some of those people, even though words will never be enough.

To begin, I would like to thank Dr. Matthew Price, Dr. David Boehm, Dr. Kimberly Woznack and Dr. Ali Sezer. At Cal U, these extraordinary educators were instrumental in where I am today. I would also like to thank the Chemistry and Biology departments at Cal U. Without their individualized, rigorous teaching I don't feel that I would've been able to enter graduate school as prepared as I was. I am deeply grateful for all the experiences and opportunities awarded to me throughout my undergraduate career.

Above all, I am exceptionally grateful to have an advisor like Dr. Justin Legleiter. His humor, sense of self and personality made my graduate experience truly enjoyable. During the more turmoiled times of my degree progression, Dr. Legleiter was always encouraging, friendly, and his gentle discipline inspired me in my own work. He gave me invaluable opportunities for exploration of techniques, collaborations within my work, and was always available to answer seemingly endless experimental questions that arose during my experiments. I feel truly fortunate to have an advisor who I felt seen by and one who truly enjoys his craft and wants to share that joy with his students and the scientific community. I don't think words could describe how grateful I am for his wonderful patience, motivation, and mentorship throughout my time at West Virginia University. He is truly one of the kindest, most hardworking individuals I know. 
I would like to thank West Virginia University and the C. Eugene Bennett Department of Chemistry for providing me with a doctoral scholarship for the duration of my degree, and the additional funding agencies including $\mathrm{NIH}$, who funded this work. I would also like to thank my committee members in my own way, as each of them I also attribute to my success. To Dr. Stephen Valentine, I would like to express my thanks for your kindness over the years, my admiration for your work ethic and the opportunity to participate in your environmental course. That course inspired me to think deeply and do research on the impact we have on the planet just by going about our daily lives. To Dr. Kenneth Showalter, I would like to express my gratitude for your helpful advice at times over the years, your daily kindness and your encouragement when I was struggling with coursework or exams. To Dr. Blake Mertz, you have always inspired me to incorporate some fun into my workday, as you always were very calm and responsible but still ready for an adventure. I thank you for your kindness, your jokes and your encouragement over the years. Finally, I would like to thank Dr. Visvanathan Ramamurthy. Not only for the help and guidance in the beginnings of the phosphorylation project, but also for helping to shape my confidence right from the start in your teachings. Your method of instruction and guidance in the lab really resonated with me. Though I only spent a small amount of my time working on the HSC campus, the skills I learned carried me through my degree. It has been a joy to collaborate with your lab, and I am grateful for that opportunity. I would also like to thank Dr. Gregory Dudley and Dr. Erin Battin, for allowing me to collaborate with them to expand my skill sets in addition to my studies. Finally, I would like to thank my lab mates past and present for all of the assistance, guidance, encouragement and friendship. 
The biggest thanks of all goes to my family. I want to thank my mom and my dad for the unwavering support and encouragement through the years. I would not be where I am today, and nothing I have achieved would've been possible without you both. I want to thank you for always believing in me. I want to thank you for instilling in me a strong work ethic, an inability to give up, and a strong will to match a strong voice. Even when I did not want to move on, you both helped me to always see the bigger picture and keep going. Words could never express my gratitude for how I was raised, and for you both to push and believe in me. Even if I couldn't ever have that chemistry set that I always wanted as a kid, you always encouraged my passions and outlets of creativity and now those passions have awarded me a lifelong career. I also would like to thank my brother and sister, my grandparents, and my aunts and uncles. I am more fortunate than most to have a huge family and with that, a huge support system full of love. A special thank you goes to my late grandmother, who sadly did not get to see this journey end but was there to see it begin and told me I could do it.

I also would like to thank the awesome friends I have made during this journey. To Dr. Maryssa Beasley, Nicolas Frazee, Blaine McClay, Paymon Doroodian, and Beka Krupa, you all have made lasting impressions on me and I hope that we continue to band together no matter where our lives take us. The adventures we had were never short of refreshing, and it certainly helped when any of us were struggling. An incredibly special thank you goes to my favorite collaborator, Dr. Maryssa Beasley. You were my go-to in and out of the lab, my voice of reason and sanity, my hype-woman and above all, an amazing friend. You taught me the perfect balance of work and play, how to look 
at life with a little more sunshine and introduced into my life to three more cheerleaders: your wonderful parents and Paymon.

In closing, I would like to finally thank my partner, and my son. To Brian, we started this journey together and withstood many trials and tribulations, and it's amazing how fast it's gone. You have always had faith in me and still continue to. Through sleepless nights, tears, and fears, you were always there. Your unconditional love and support have been a pillar of strength for me throughout this journey and continues throughout our relationship as we navigate parenthood. I wouldn't have made it without you, and I thank you for being my therapist, my place to come home to, and most importantly, my best friend. To Ethan, my son, I thank you for making me a mother, specifically during graduate school. I have never known stronger discipline, efficiency and determination than what I have now that you are in my life. You have helped me to see things that seemed overwhelming in graduate school, are simply obstacles to the best life I can make for you. You have opened my eyes to so many things, and the stress and timelines I have been subjected to I would do again. Being your mother has been my greatest joy, and I dedicate everything I have done, to you. 


\section{Table of Contents}

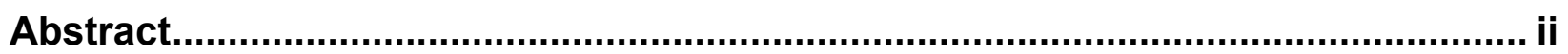

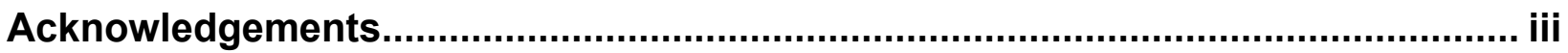

Table of Contents ............................................................................................ viii-xi

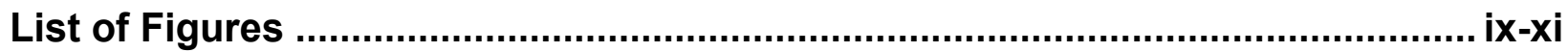

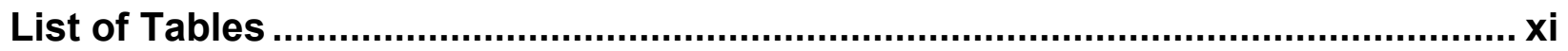

List of Symbols and Abbreviations ............................................................ xii-xiii

1. Introduction: Role of the Structure and Functions of the Huntingtin Protein Related to Huntingtin's Disease......................................................................... 1-26

1.1 General Protein Misfolding Diseases .............................................................. 1

1.2 Huntington's Disease and the Role of the Huntingtin Protein ............................... 3

1.3 Mechanisms and Toxicity of Aggregation ................................................... 5

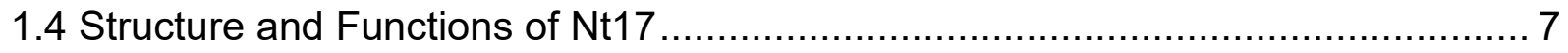

1.5 Post Translational Modifications within Nt17 …........................................... 9

1.6 Significance of Lipid Interactions with Huntingtin Protein ................................ 12

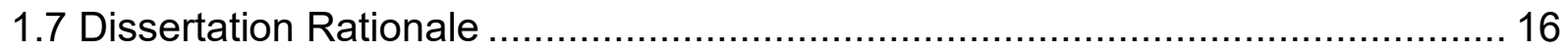

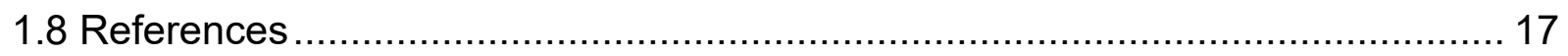

2. Phosphomimetic Mutations Impact Huntingtin Aggregation in the Presence of a Variety of Lipid Systems........................................................................... 27-63

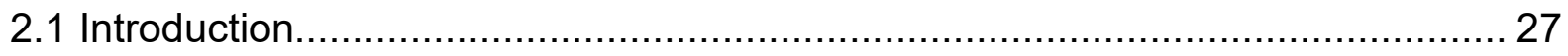

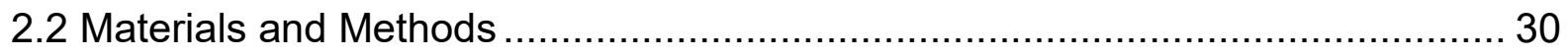

2.2.1 Preparation of Phosphomimetic Constructs.......................................... 30

2.2.2 Expression, Purification of (GST)-htt-exon1 Fusion Protein ..................... 30

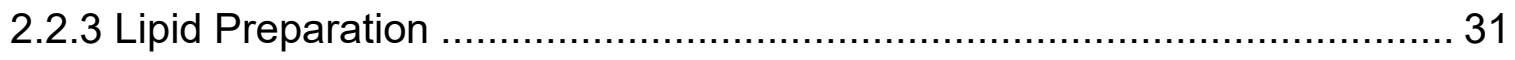

2.2.4 Thioflavin T (ThT) Aggregation Assay ............................................... 31

2.2.5 Polydiacetylene (PDA) Vesicle Binding Assay ......................................... 31 
2.2.6 Atomic Force Microscopy (AFM) …............................................... 32

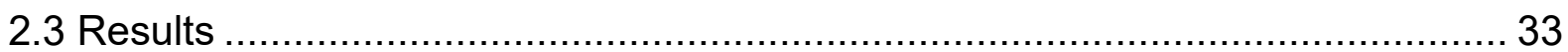

2.3.1 Phosphomimetic mutations in Nt17 alter htt aggregation ........................ 33

2.3.2 Phosphomimetics of htt alter aggregation in the presence of lipids ........... 38

2.3.3 Phosphomimetics of htt impact binding in a lipid specific manner ............ 46

2.3.4 Phosphomimetics of htt influence disruption of lipid bilayers ................... 48

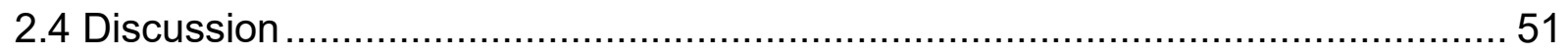

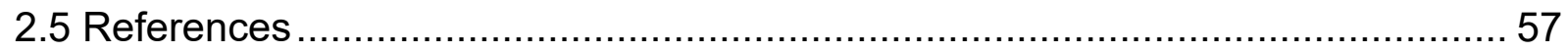

3. Macromolecular Crowding Alters Huntingtin Aggregation at Interfaces ...... 64-95

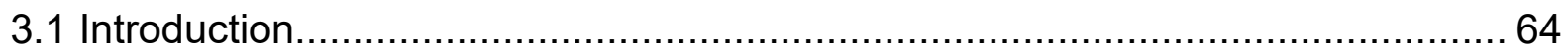

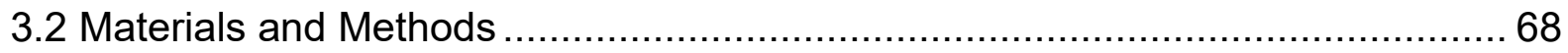

3.2.1 Expression, Purification of (GST)-htt-exon1 Fusion Protein ..................... 68

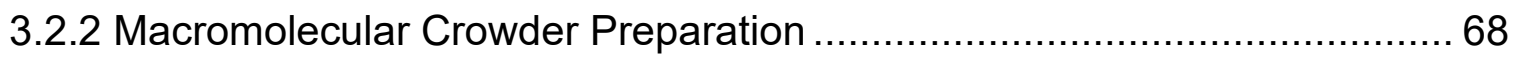

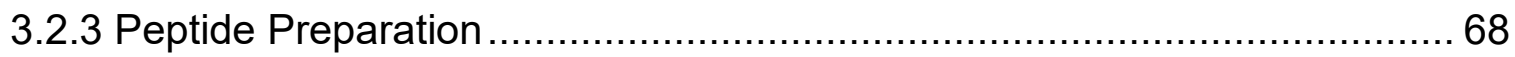

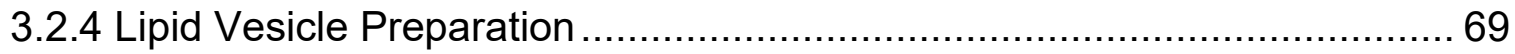

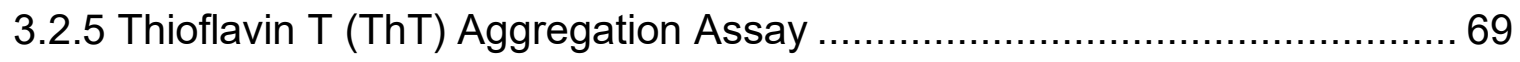

3.2.6 Polydiacetylene (PDA) Vesicle Binding Assay ....................................... 69

3.2.7 Atomic Force Microscopy (AFM) ……............................................ 70

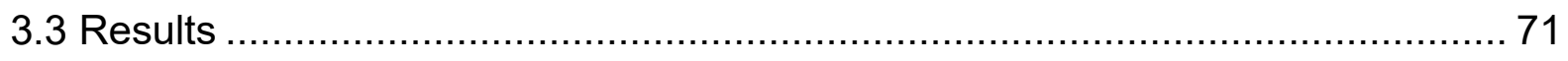

3.3.1 Crowding alters htt aggregation in bulk solution ................................. 71

3.3.2 $\mathrm{Htt}$ aggregation at a solid/liquid interface is modified by macromolecular

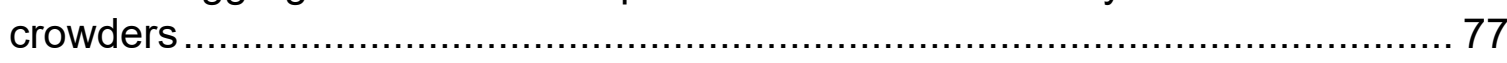

3.3.3 $\mathrm{Htt}$ aggregation on lipid bilayers is modified by macromolecular crowders.

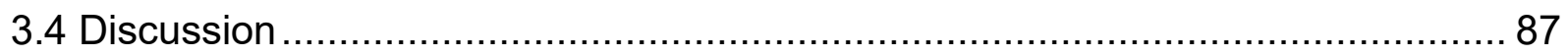


4.1 Impacts of PIP Lipids and Htt on Membranes

4.2 Exploration of PTM Crosstalk, Charged and Complex Lipid Systems and Organelles with $\mathrm{Htt}$

4.3 Liquid/liquid Phase Separations, Stress Granules, and Htt Aggregation Mechanisms In Vivo

4.4 References. 106

Appendix: Huntingtin Protein Purification and Experimentation with Macromolecular Crowders: An Innovative Undergraduate Biochemistry Experiment.

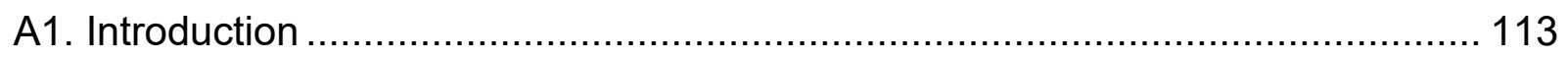

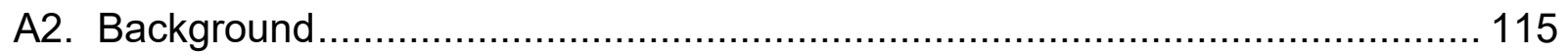

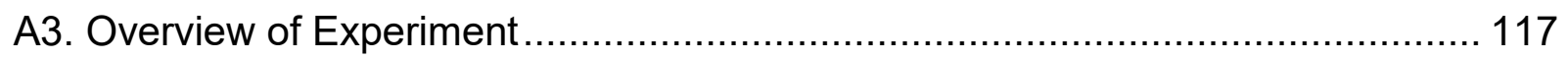

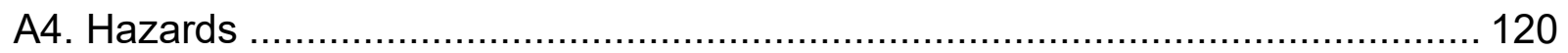

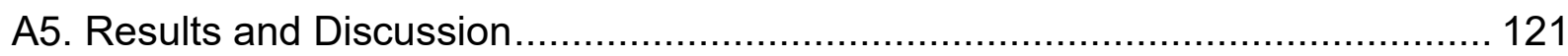

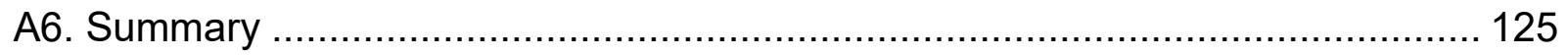

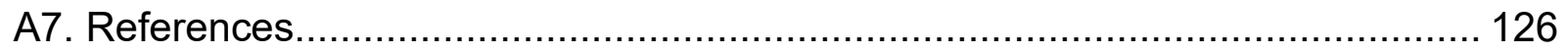

A8. Brief Overview of Additional Information for Instructors ................................. 128

A8.1 Lab Summary for Instructors ...................................................... 128

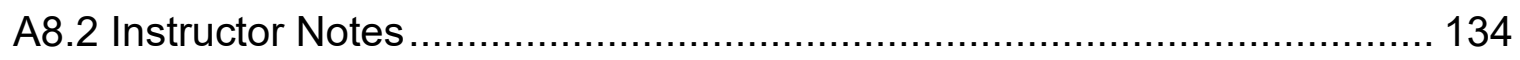

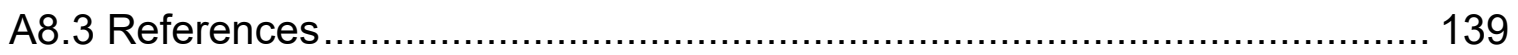

List of Figures

1. Introduction: Role of the Structure and Functions of the Huntingtin Protein Related to Huntingtin's Disease .................................................................... 1

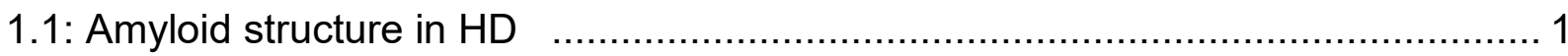

1.2: Protein folding funnel energy landscape ................................................. 3 
1.3: Huntingtin protein, exon1 and polyQ thresholds ......................................... 4

1.4: Schematic model of htt aggregation pathways .............................................. 6

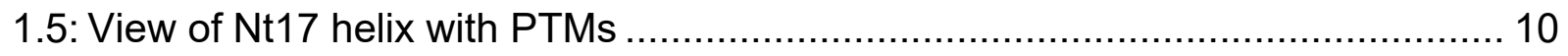

1.6: Mechanism of membrane binding interactions of htt ..................................... 13

2. Phosphomimetic mutations impact huntingtin aggregation in the presence of a

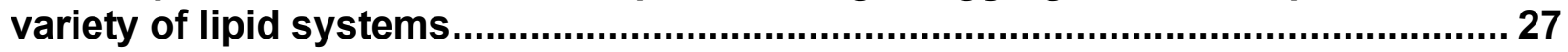

2.1: The impact of phosphomimetic mutations on htt aggregation ........................... 35

2.2: Phosphomimetic mutations influence htt aggregation in the presence of lipid vesicles

2.3: Representative AFM images of backgrounds associated with deposited solutions of lipid vesicles.

2.4: Phosphomimetic htt constructs form a variety of aggregates in the presence of lipid vesicles

2.5: Phosphomimetic construct aggregates observed with ex situ AFM in the presence of TBLE .

2.6: Phosphomimetic construct aggregates observed with ex situ AFM in the presence of POPC .

2.7: Phosphomimetic construct aggregates observed with ex situ AFM in the presence of POPG. 44

2.8: Lipids promote unique $h t t$ and phosphomimetic htt oligomer morphologies 45

2.9: Phosphomimetic mutations in htt alter its ability to bind a variety of lipid vesicles.

2.10: Phosphomimetic mutations alter htt aggregation on the surface of a TBLE bilayer ......

3. Macromolecular Crowding Alters Huntingtin Aggregation at Interfaces

3.1: The impact of macromolecular crowders on htt-exon1(46Q) fibril formation as assessed by a ThT assay

3.2: Ex situ AFM analysis of htt-exon1(46Q) aggregates formed in the presence of $100 \mathrm{mg} / \mathrm{ML}$ macromolecular crowders 
3.3: Representative AFM images of mica exposed to (A) neat buffer or $100 \mathrm{mg} / \mathrm{mL}$ of either (B) dextran, (C) ficoll, or (D) PEG ....................................................... 76

3.4: Ex situ AFM analysis of htt-exon1(46Q) aggregates formed in the presence of 50

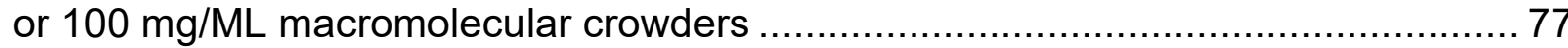

3.5: Tracking of individual Nt17-Q35-P10-KK aggregates in mica as a function of time with no crowders in the aqueous phase 78

3.6: Comparison of Nt17-Q35-P10-KK aggregation on mica with different macromolecular crowders in the aqueous phase

3.7: The impact of macromolecular crowders on htt-exon1(46Q) interaction with TBLE

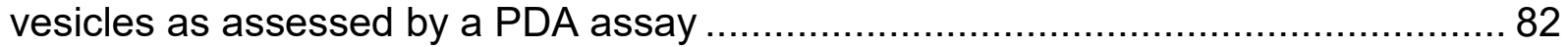

3.8: Control TBLE/PDA lipid binding assays assessing if macromolecular crowders

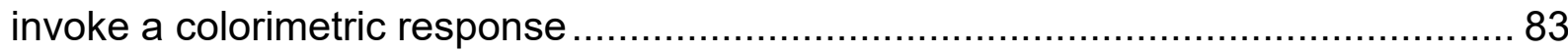

3.9: The impact of macromolecular crowders on htt-exon1(46Q) fibril formation in the presence of TBLE vesicles as assessed by a ThT assay.....

3.10: Comparison of htt-exon1(46Q) aggregation on supported TBLE bilayers with different macromolecular crowders in the aqueous phase ...................................... 85

4. Future Work and Concluding Remarks ……........................................................ 96

4.1: Proposed interaction of Nt17 association with PIP and PIP/Ca ${ }^{+2}$ vesicles .......... 99

4.2: Comparison of phosphorylation and acetylation PTM sites on Nt17 ............... 101

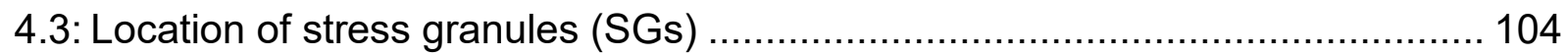

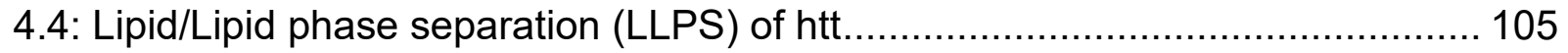

Appendix: Introduction: Role of the Structure and Functions of the Huntingtin Protein Related to Huntingtin's Disease .............................................................. 113

A1: Representation of student obtained data during experiment ......................... 122 List of Tables

Table A1: Devised Solutions to Traditional Experimental Setbacks 120 


\section{List of Symbols and Abbreviations}

\begin{tabular}{|c|c|}
\hline a-syn & a-synuclein \\
\hline$A \beta$ & $\beta$-amyloid peptide \\
\hline ACS & American Chemical Society \\
\hline$A D$ & Alzheimer's Disease \\
\hline $\mathrm{AH}$ & Amphipathic $\alpha$-helix \\
\hline AFM & Atomic Force Microscopy \\
\hline AKT & Protein Kinase B \\
\hline BSL-1 & Biological Safety Level-1 \\
\hline CR & Colorimetric Response \\
\hline DOPG & 1,2-dioleoyl-sn-glycero-3-phosphoglycerol \\
\hline DRPLA & Dentatorubral-Pallidoluysian Atrophy \\
\hline E. Coli & Escherichia Coli \\
\hline EDTA & Ethylenediaminetetraacetic acid \\
\hline EPR & Electron Paramagnetic Resonance \\
\hline ER & Endoplasmic Reticulum \\
\hline ERAD & $\begin{array}{l}\text { Endoplasmic-Reticulum-Associated } \\
\text { Protein Degradation }\end{array}$ \\
\hline Exon1 & First exon of the huntingtin protein \\
\hline GST & Glutathione-S-Transferase \\
\hline $\mathrm{HCl}$ & Hydrochloric Acid \\
\hline HD & Huntington's Disease \\
\hline HFIP & Hexafluoroisopropanol \\
\hline $\mathrm{Htt}$ & Huntingtin Protein \\
\hline Htt-Exon1 & Huntingtin exon1 \\
\hline Htt-Exon1(46Q) & $\begin{array}{l}\text { Huntingtin exon } 1 \text { with } 46 \text { glutamine } \\
\text { repeats }\end{array}$ \\
\hline IDRs & Intrinsically Disordered Regions \\
\hline IPTG & Isopropyl- $\beta$-D-thiogalactoside \\
\hline LC & Liquid Chromatography \\
\hline LLPS & Liquid/liquid Phase Separation \\
\hline LUV & Large Unilamellar Vesicles \\
\hline MLOs & Membraneless Organelles \\
\hline
\end{tabular}




\begin{tabular}{|c|c|}
\hline $\mathrm{NaOH}$ & Sodium Hydroxide \\
\hline Nt17 & $\begin{array}{l}\text { The First } 17 \mathrm{~N} \text {-Terminal Amino Acids of } \\
\mathrm{Htt}\end{array}$ \\
\hline PEG & Polyethylene Glycol \\
\hline PD & Parkinson's Disease \\
\hline PDA & Polydiacetylene \\
\hline PIPs & Phosphatidylinositol Phosphates \\
\hline PolyQ & Polyglutamine \\
\hline PolyP & Polyproline \\
\hline POPC & $\begin{array}{l}\text { 1-palmitoyl-2-oleoyl-sn-glycero-3- } \\
\text { phosphocholine }\end{array}$ \\
\hline POPG & $\begin{array}{l}\text { 1-palmitoyl-2-oleoyl-sn-glycero-3- } \\
\text { (phospho-rac-(1-glycerol)) }\end{array}$ \\
\hline POPS & $\begin{array}{l}\text { 1-palmitoyl-2-oleoyl-sn-glycero-3- } \\
\text { phospho-L-serine }\end{array}$ \\
\hline PTMs & Post Translational Modifications \\
\hline Q & $\begin{array}{l}\text { One letter abbreviation for the amino acid } \\
\text { glutamine }\end{array}$ \\
\hline RMS & Root Mean Squared \\
\hline SBMA & Spinal-bulbar Muscular Atrophy \\
\hline $\begin{array}{l}\text { SCA-1, SCA-2, SCA-3, SCA-6, SCA-7, } \\
\text { SCA-12, SCA-17 }\end{array}$ & Spinocerebellar Ataxia diseases \\
\hline SEM & Standard Error of the Mean \\
\hline SGs & Stress Granules \\
\hline ssNMR & Solid-State Nuclear Magnetic Resonance \\
\hline STEM & $\begin{array}{l}\text { Science, Technology, Engineering and } \\
\text { Mathematics }\end{array}$ \\
\hline TBLE & Total Brain Lipid Extract \\
\hline ThT & Thioflavin T \\
\hline TRDs & Trinucleotide Repeat Disorders \\
\hline
\end{tabular}




\section{Introduction: Role of the Structure and Functions of the Huntingtin Protein Related to Huntingtin's Disease}

\subsection{General Protein Misfolding Diseases}

A hallmark of several neurodegenerative disorders is the systematic buildup of micron sized proteinaceous material in tissues and cellular compartments. These diseases include Parkinson's Disease (PD), Alzheimer's Disease (AD) and Huntingtin's Disease (HD). Clinical symptoms include impaired cognitive function and motor control and pathological changes comprise of synaptic abnormalities and neuronal cell loss. ${ }^{1-3}$ In each of these diseases, a hallmark is the conformational change of specific proteins inducing aggregation and accumulation of amyloids within the brain. ${ }^{4-6}$ These insoluble amyloids are identified through their cross- $\beta$ sheet rich structures and fibril morphologies. ${ }^{7,8}$ These general amyloid disorders are also classified as protein misfolding diseases, in reference to the misfolding of the normal, functional protein (Fig. 1).

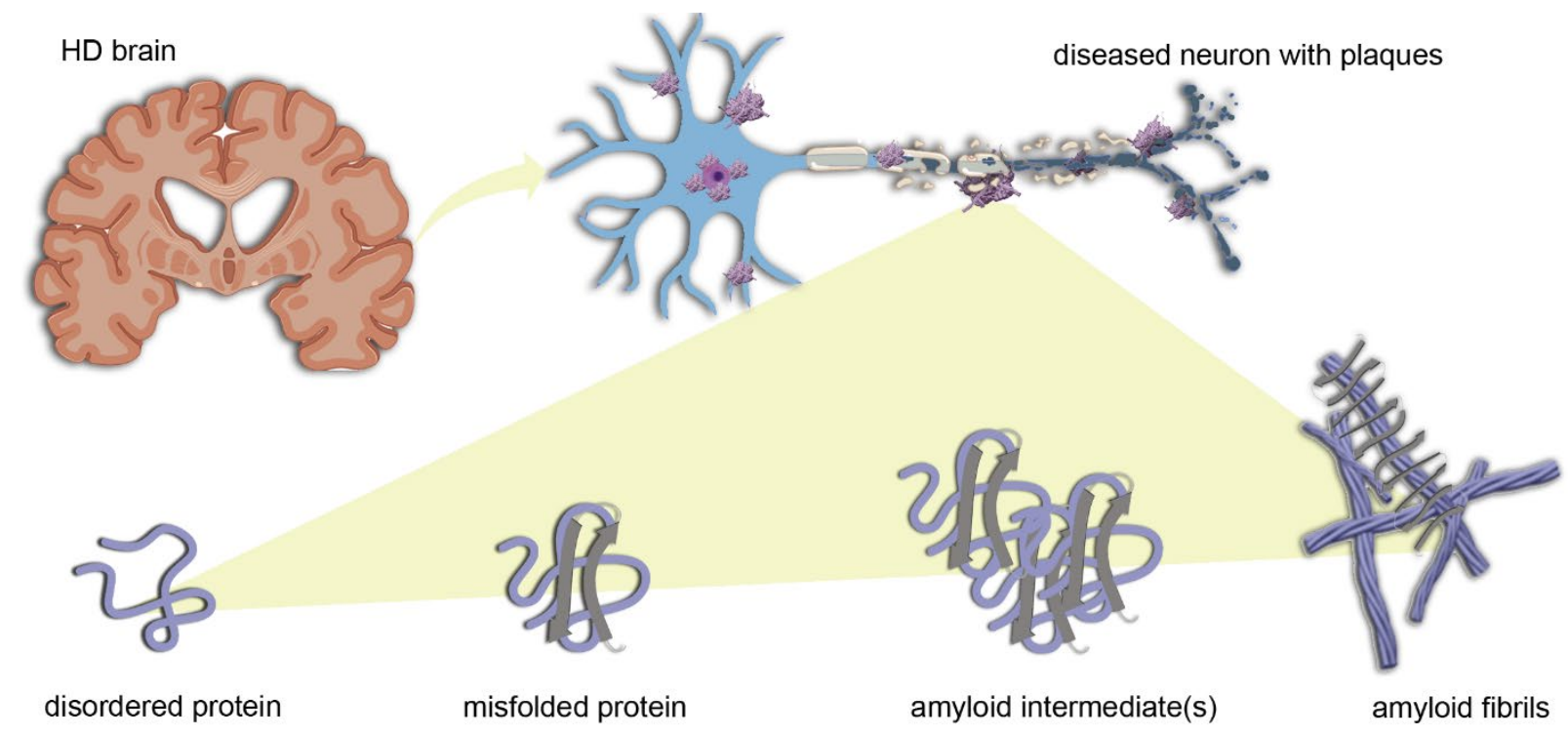


Figure 1.1: Amyloid structure in HD. Diseased neurons within HD patients' brains contain amyloid plaques which are comprised of a variety of structures stemming from the disordered protein, leading eventually to amyloid fibrils. These plaques are localized to the nucleus of the neuron. Beginning with the disordered protein, when misfolded, $\beta$-sheet structures (as shown by grey arrows) start to form. The misfolded proteins reach a critical nucleus and form an amyloid intermediate that eventually forms the $\beta$-sheet rich amyloid fibrils. Created in part with BioRender.com

The driving force behind protein folding is the tendency of hydrophobic residues to sequester together within the protein interior due to a phenomenon known as the hydrophobic effect. ${ }^{9}$ Functional proteins are newly synthesized from linear chains of amino acids and conform to a well-defined three dimensional compact structure of minimal energy, consistent with the concept of a folding energy landscape funnel (Fig. 2). ${ }^{10}$ In this compact, native conformation, the hydrophilic residues are located on the protein surface and exposed to water. The pathway along the energy landscape leading to the native conformation contains local energy minima in which partially folded intermediates can become trapped. ${ }^{11-13}$ These intermediates risk misfolding and following alternative folding pathways leading to structures differing from the native conformation. In amyloidogenic proteins, these alternative intermediates lead to $\beta$-sheet rich monomeric or multimeric structures such as oligomers that can further promote the formation of fibrils, or amorphous aggregates. ${ }^{14}$ These altered conformations associated with amyloid result in affected biological properties and a toxic gain of function. ${ }^{15,16}$ 


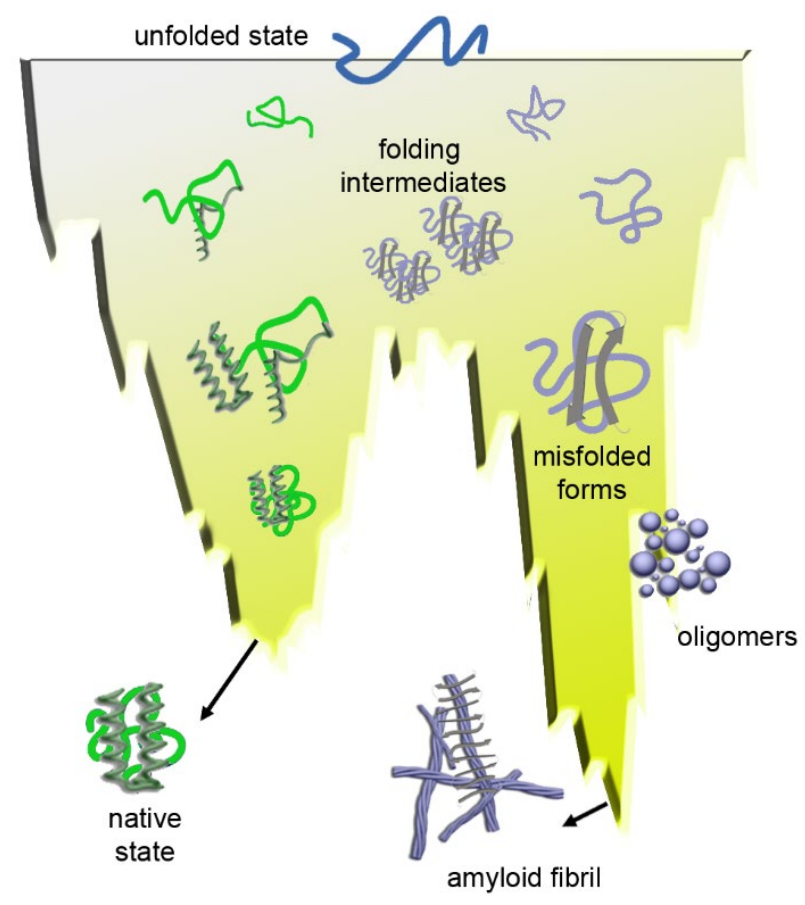

Figure 1.2: Protein folding funnel energy landscape. Unfolded proteins generally undergo a variety of conformations to react the most compact and stable native state (green pathway). If the unfolded protein proceeds through an alternate pathway other than the native folding pathway by becoming trapped in energy minima, misfolded intermediates are formed (purple pathway). For amyloid proteins, these misfolding intermediates are $\beta$-sheet rich and continue to form amyloid aggregates of lower energy, with increasing $\beta$-sheet content. Created in part with BioRender.com

\subsection{Huntington's Disease and the Role of the Huntingtin Protein}

Huntington's Disease (HD) is a fatal, autosomal dominant neurodegenerative disease that results in dystonia, chorea, and loss of cognitive abilities. ${ }^{17,18}$ This disorder results from a mutation in the HTT gene found on the short arm of chromosome 4, which codes for the huntingtin protein (htt). The mutation is a CAG trinucleotide expansion, which codes for the amino acid glutamine (Q). Therefore, the expansion in the translated huntingtin (htt) protein is referred to as the polyglutamine (polyQ) domain, and this domain is located near the N-terminus of htt. ${ }^{19} \mathrm{HD}$ is one of ten identified CAGrepeat or polyQ disorders. In these diseases, including HD, there is a high correlation between age of onset, polyQ length, and aggregation of proteins containing expanded 
polyQ. ${ }^{20,21}$ While the polyQ domain is normally $\sim 17-20$ residues long in htt, a poly $\mathrm{Q}$ repeat length of $\sim 35$ is the critical threshold associated with HD, and disease severity increases as the polyQ length expands (Fig. 3). ${ }^{19,22-25}$

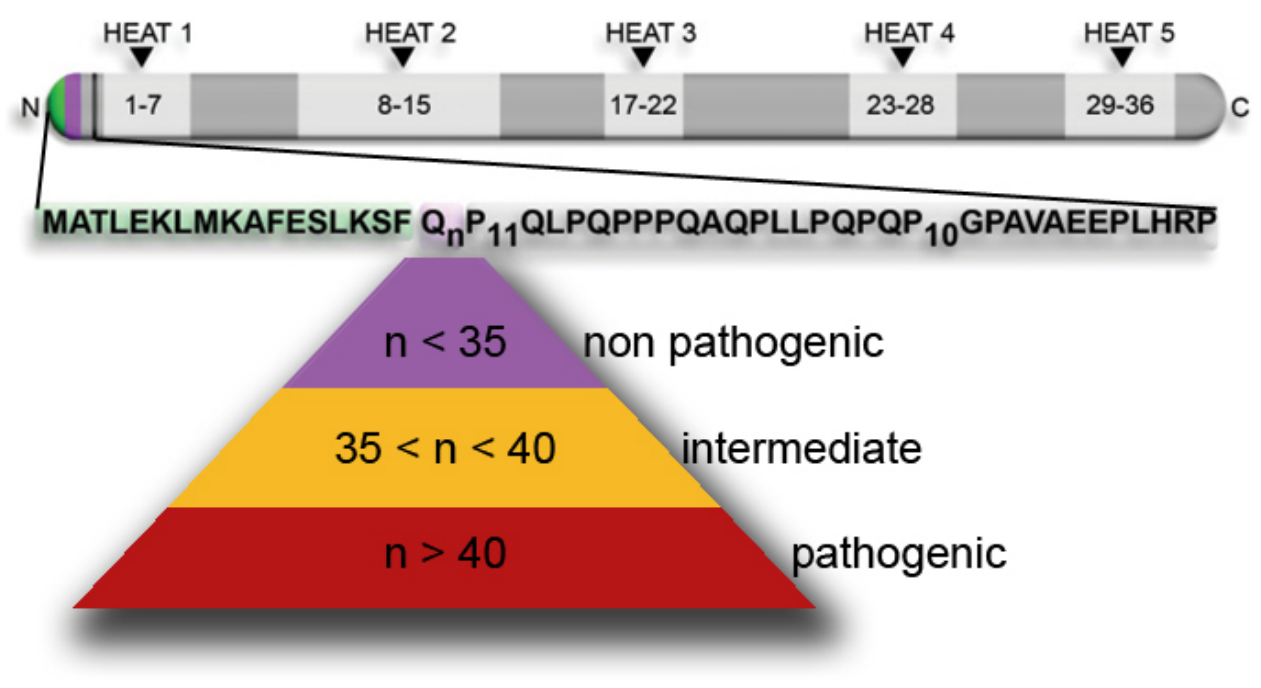

Figure 1.3: Huntingtin protein, exon1 and polyQ thresholds. An abnormal trinucleotide (CAG) repeat length within the IT15 gene is the root cause of htt's pathogenicity. The first exon at the N-terminus, known as exon1, contains three distinct domains, Nt17 (green), polyQ (purple) and a proline rich domain (polyP) (grey). As the polyQ length increases, age of onset decreases, with the intermediate region indicating that disease will occur later in life (triangle).

The exact mass of htt is dependent upon the size of the polyQ domain, however, the full length of htt is comprised of 3144 amino acids, 67 exons and is $\sim 350 \mathrm{kDa} .{ }^{19}$ The polyQ domain follows is located in the first exon of htt and is preceded by 17 amino acids commonly referred to as $\mathrm{Nt17}$. Beyond Nt17, the polyQ domain is flanked in exon1 on the C-terminal side by a proline-rich domain that contains two polyproline (polyP) stretches. The flanking domains have varying roles. Nt17 initiates lipid binding and aggregation, ${ }^{26-30}$ and the polyP domain modulates htt-lipid interaction ${ }^{26}$ and htt nucleation. ${ }^{31}$ Evidence suggests that $\mathrm{N}$-terminal fragments comparable to exon 1 are directly involved in $\mathrm{HD},{ }^{32-37}$ thus many studies have been completed with biologically relevant $\mathrm{N}$-terminal htt fragments. Htt-exon1 is responsible for increased aggregation 
and accrual of proteinaceous material in the striatum in HD patients, ${ }^{38}$ and it is a stable fragment that is continuously produced in the brain through proteolysis of full-length htt. ${ }^{32} \mathrm{~N}$-terminal fragments comparable to htt-exon1 are detected in knock-in mouse models expressing full length $\mathrm{htt}^{32}$, resulting in similar progressive neurological phenotypes and age-dependent accumulation. ${ }^{33,38,39}$ Evidence suggest the use of httexon1 is an ideal model for physiologically relevant, in vitro biophysical studies of oligomers and fibrils formed by purified htt-exon1, as htt-exon1 is responsible for increased aggregation and accrual of proteinaceous material in the striatum in $\mathrm{HD}$ patients. ${ }^{38}$

\subsection{Mechanisms and Toxicity of Aggregation}

Similar to other neurodegenerative diseases, fibrils in HD form via a nucleation dependent polymerization growth pathway. Though the details of aggregation fluctuate for each protein, proteins containing expanded polyQ tracts tend to follow the basic polyQ-mediated aggregation schemes. ${ }^{14}$ Mutant htt assembles into a collection of various aggregates associated with amyloid diseases, such as fibrils, oligomers, annular structures, inclusion bodies and amorphous aggregates. ${ }^{20,40-42}$

A rate limiting ${ }^{43,44}$ nucleation phase, known as the lag phase, is associated with the alteration of the native structure of the protein to non-native conformations. ${ }^{45} \mathrm{~A}$ mono- or multimeric nucleus formed in the lag phase through several inter- and intramolecular forces causes the rapid elongation of $\beta$-sheet rich fibrillar structures. ${ }^{44,46,47}$ There are two dominant aggregation pathways of generic polyQ aggregation: a) direct nucleation from monomer to fibril ${ }^{48}$ and b) the reordering of soluble oligomeric structures into $\beta$-sheet rich fibrillar structures. ${ }^{42}$ These two 
mechanisms are not mutually exclusive; however, conditions that promote oligomerization as the dominant or "on pathway" aggregation pathway accelerate the process. ${ }^{14}$

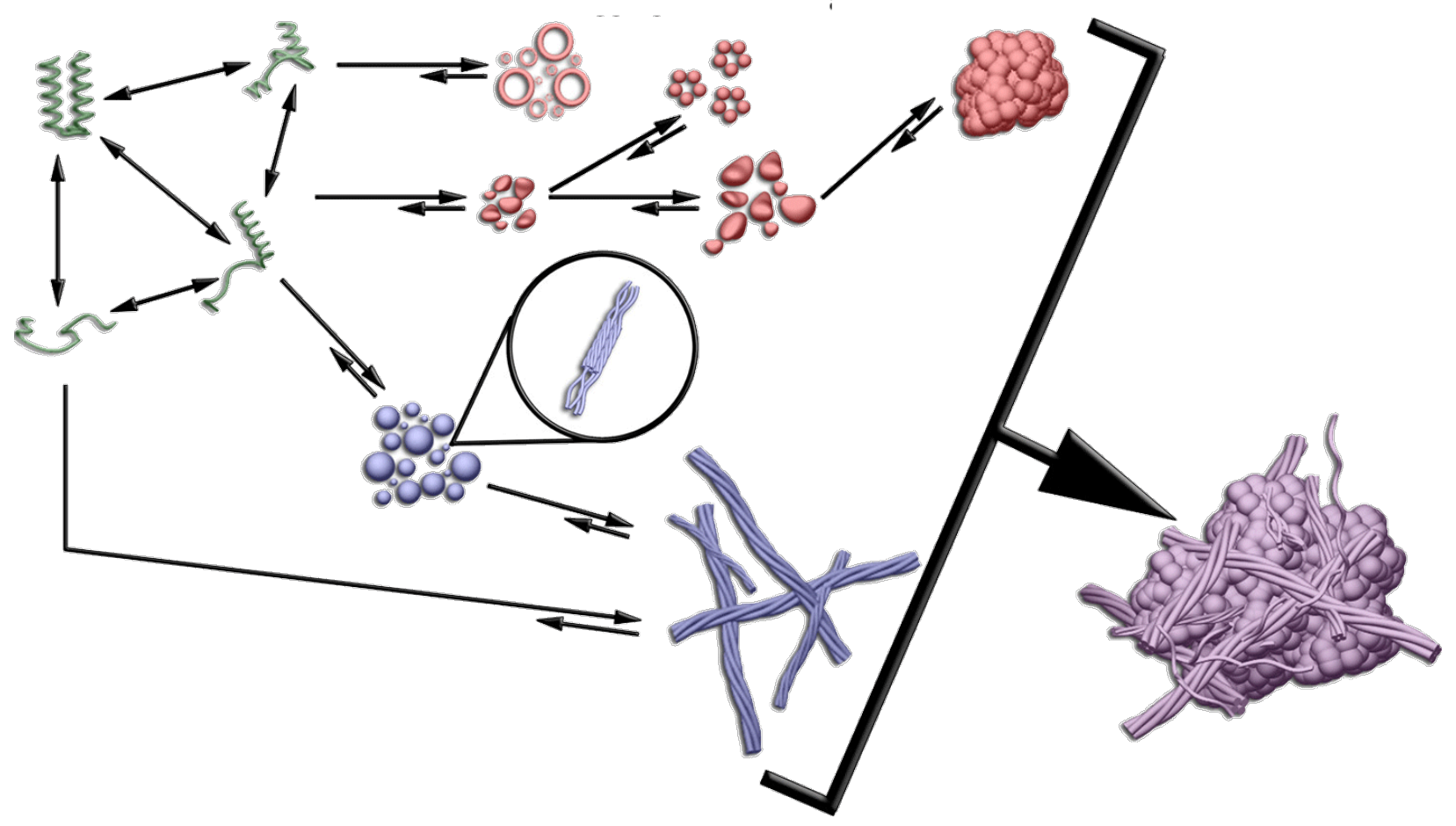

Figure 1.4: Schematic model of htt aggregation pathways. Htt aggregation follows three main aggregation pathways, which begin with a misfolded, non-native conformation of a monomer (green). On pathway mechanisms eventually lead to the formation of $\beta$-sheet rich fibrils, by direct transition of misfolded monomer to fibril (bottom), or through a nucleation mechanism resulting in oligomeric species (second from bottom, circle). Off-pathway mechanisms include the formation of oligomers, amorphous and annular aggregates (top and second from top, pink). These pathways are not mutually exclusive, and together the various aggregates can form large inclusions (bottom right) seen in HD patients' brains. Reprinted from Adegbuyiro, et al. Proteins Containing Expanded Polyglutamine Tracts and Neurodegenerative Disease. Copyright 2017 American Chemical Society.

The aggregation mechanisms can result in an assortment of aggregates coexisting, but there is limited knowledge of the exact toxicity associated with each specific aggregate species. This heterogeneity dictates the need for determining the exact aggregation mechanism, defining structural elements related with the toxic forms of htt aggregates and highlights the intricacy of mutant htt toxicity. Debate continues 
regarding the focal cause of toxicity associated with htt aggregates. Inclusion body formation, while the hallmark of $\mathrm{HD}$, may signify a protective cellular response to

eliminate diffuse htt aggregates, which have been reported to be the acutely toxic. ${ }^{47}$ The monomeric form of htt-exon1 encompasses intrinsically disordered domains that interact intramolecularly to encourage toxic oligomerization of htt. ${ }^{49-51}$ Soluble oligomers can vary in stability and size, ranging from small dimers and tetramers to larger octamers and dodecamers. Htt has been known to form a variety of oligomers, as observed by AFM (atomic force microscopy), which can initiate htt fibril formation in vitro. ${ }^{34,44}$ Oligomers morphologically and biochemically indistinguishable from those observed in vitro are detectable in transgenic cells expressing htt, HD mouse models, and patients' brains. ${ }^{50,52}$

Particular attention has been fixated on how flanking sequences on either side of the polyQ domain promote certain aggregation mechanisms. Addition of polyP residues to the C-terminus forces a PPII-like structure into the polyQ domain, which competes against the aggregation-prone $\beta$-sheet. ${ }^{53} \mathrm{Nt} 17$ stimulates aggregation by encouraging formation of helical oligomers. ${ }^{26,53}$ The oligomer structure brings polyQ domains into closer proximity and lowers the energy barrier to nucleate $\beta$-sheet formation. ${ }^{53}$ This key role of Nt17 in the upstream stages of htt aggregation suggests that it is a viable therapeutic target.

\subsection{Structure and Functions of $\mathrm{Nt17}$}

Understanding the structural dynamics of Nt17 would provide a deeper understanding to the complexity of htt aggregation. Nt17 in ionic buffer is intrinsically disordered but demonstrates the ability to undergo conformational transitions leading to 
an amphipathic $\alpha$-helix $(\mathrm{AH})$ when interacting with binding partners. ${ }^{20,30}$ The structure of an $\mathrm{AH}$ is favorable for establishing a collection of helix bundles in aqueous environment, driven by maximizing the interaction of the hydrophobic faces of adjacent helices. ${ }^{29,54}$ The formation of these interacting helices has been anticipated to play a role in the preliminary stages of htt aggregation. Through ordering into helical bundles, Nt17 promotes the stability of aggregates and an oligomeric intermediate aggregation pathway, leading to an increased rate of aggregation. ${ }^{55-57}$ Oligomers are a potentially critical toxic species; therefore, the promotion of oligomerization by Nt17 could have potentially important outcomes in htt-related toxicity. Intermolecular interactions leading to the self-association of $\mathrm{Nt} 17$ enables the formation of these helical oligomers, leading to $\beta$-sheet rich fibrils associated with the polyQ core; however, Nt17 maintains its $\mathrm{AH}$ structure in the fibril. ${ }^{26}$

Peptides containing polyQ but lack the $\mathrm{N}$-terminal flanking sequence tend not to form oligomeric intermediates, thus proceeding directly to fibrillar aggregates. ${ }^{26,30}$ However, pure polyQ-peptide aggregating on a surface have been discovered to form oligomers. ${ }^{33,54}$ Free Nt17, which lacks the polyQ domain, has been demonstrated to inhibit formation of $\mathrm{Nt}_{17} \mathrm{Q}_{37} \mathrm{P}_{10} \mathrm{KK}$ peptides in vitro. ${ }^{26,30}$ The mechanism appears to be identical to the mechanism by which nucleation occurs, by increasing the distance between adjacent polyQ tracts and the energy for polyQ fibrillization to occur. ${ }^{58}$ Steric hinderances can also interfere with the ability of Nt17 to promote oligomerization. In cases where a Myc-tag preceded Nt17 in full length exon1, the formation of oligomers in vitro was reduced without changing the rate of fibril formation when equated to similar proteins that lacked the Myc-tag. ${ }^{20}$ Increased structural complexation using small 
molecules, antibody-antigen, or molecular chaperones that interacts directly with this domain are also practical means of inhibiting $\mathrm{N}$-terminal aggregation. ${ }^{3,59-62}$

Membrane binding AHs is a common motif in proteins and peptides, with Nt17 demonstrating these functions. ${ }^{28} \mathrm{AH}$ lipid binding begins with separation of the hydrophilic and hydrophobic sides of the AH structure. Hydrophilic residues are solvent exposed and accessible for multiple binding partners, while hydrophobic residues are buried in the acyl chain of the lipid membrane, leading to an $\mathrm{AH}$ structure parallel with the surface. ${ }^{28,63,64}$ This binding approach is driven by long-range electrostatic interactions between the peptide and lipid headgroups coupled with conformational rearrangements of the peptide that allow the hydrophobic residues to remain closer to the membrane core. ${ }^{64}$

Supplementary $\mathrm{AH}$ abilities of $\mathrm{Nt}-17$ function in the preliminary insertion of poly $\mathrm{Q}$ peptides into a lipid membrane structure, which can increase the local concentration of aggregation-prone polyQ region. This interaction strengthens the reactions between polyQ domains through a 2-dimensional diffusion on the membrane surface. ${ }^{54,65,66} \mathrm{AHs}$ also have several practical features, including detection of defects induced by curvature, which allows preferential sensing and binding of highly curved membranes. ${ }^{67}$ Since AHs weakly bind membranes, their interactions can be effortlessly maintained. For example, Nt17 can isolate truncated htt-exon1 peptides to regions of curvature on supported lipid bilayers. ${ }^{68}$ Detailed understanding of Nt17 lipid binding mechanisms can elucidate the methods of htt aggregation at lipid surfaces.

\subsection{Post Translational Modifications within Nt17}

Introduction | 9 
Within the Nt17 helix, there are several sites that undergo post-translational modifications (PTMs), with some sites associated with multiple PTMs. ${ }^{69-71}$ These PTMs include acetylation, ${ }^{72,73}$ oxidation, ${ }^{74}$ SUMOylation, ${ }^{69,75,76}$ ubiquitination, ${ }^{77}$ and phosphorylation. ${ }^{43,51,78-81}$ Other less known, but observed, PTMs include palmitoylation ${ }^{82}$ and transglutamination ${ }^{83}$. Collectively, these PTMs of $\mathrm{Nt} 17$ have significant effects on htt function and disease progression. Factors of htt function including translocation, ${ }^{84-86}$ membrane association and binding, ${ }^{76,81,87}$ toxicity, ${ }^{29,69,70,75,78}$ and aggregation ${ }^{76,78,81,87}$ can be traced back to specific amino acids within Nt17.

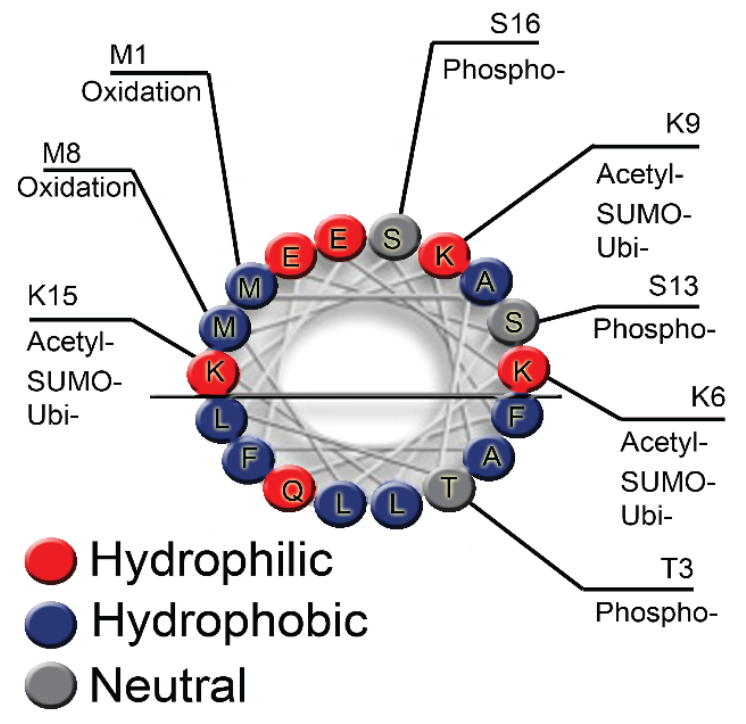

Figure 1.5: View of Nt17 helix with PTMs. The view down the top of the barrel of the Nt17 $\alpha$ helix shows hydrophobicity of each residue (as indicated by legend) and all reported PTMs. The center line represents the membrane of cells, in which all residues below the line are known to bury within the membrane due to their hydrophobic nature. Adapted in part with permission from Arndt, J.R. et al. The Emerging Role of the First 17 Amino Acids of Huntingtin in Huntington's Disease. Copyright 2015 Biomolecular Concepts.

The localization of lysine residues on the edge of the hydrophilic portion of the $\mathrm{AH}$ (K6 and K15) suggests a potential roles in stabilizing early htt oligomers. ${ }^{88}$ Three lysine residues are found within $\mathrm{Nt} 17,(\mathrm{~K} 6, \mathrm{~K} 9, \mathrm{~K} 15)$ and all are available for acetylation, 
SUMOylation, and ubiquitination. Acetylation of these residues decreases the severity of htt lipid binding, resulting membrane disruption, and promotes the formation of large globular aggregates instead of fibrils. ${ }^{87}$ Proteomic mapping via mass spectrometry confirmed K9 is significantly acetylated in mammalian cell lysates. ${ }^{72}$

SUMOylation of these lysine residues modify htt-induced pathogenesis for both cell and animal models ${ }^{69,75}$ and alter aggregation and the ability of $h t t$ to bind membranes. ${ }^{69,76}$ At $\mathrm{K} 6$ and $\mathrm{K} 9$, SUMOylation stabilized htt-exon1 fragments, effectively retarding aggregation and toxicity in Drosophila models. ${ }^{69}$ SUMOylation by Rhes, a small G protein, specially binds mutant htt over wild type by acting as a SUMO E3 ligase, increasing toxicity and soluble levels of htt. ${ }^{89}$ The natural ubiquitination process tags proteins for degradation and is known to compete for the same lysine residues as SUMOylation, and both reduce the toxicity of mutant htt. ${ }^{90}$ However, the presence of ubiquitin in htt inclusions demonstrate that this degradation mechanism becomes inefficient in HD. ${ }^{91}$ Since SUMOylation and ubiquitination compete for the same lysines, it can be implied that one method could be used to control the other. For example, serine 13 (S13) and serine 16 (S16) phosphomimics in htt were found to regulate SUMO-1 modifications in cells and in vivo. ${ }^{43,80}$ These same phosphomimics also enhance K6 htt SUMOylation, which could modulate K9 acetylation. ${ }^{75}$

There are three phosphorylation sites within the Nt17 of htt-exon1, S13, S16 and threonine 3 (T3), with even more sites in full-length htt. Extensive studies suggest that htt phosphorylation is associated with reduced levels of mutant htt toxicity, aggregation, and lipid binding ${ }^{43,71,78,80,81,92,93}$ Phosphorylation at $\mathrm{S} 13$ or $\mathrm{S} 16$ reduce helical content. ${ }^{81,92}$ whileT3 phosphorylation stabilizes helical content, yet phosphorylation at all 
three sited reduced aggregation. ${ }^{94,95} \mathrm{~A}$ combination of serine phosphorylation mimics established that serine to aspartate mutations greatly reduced the stability of the fibril structure and formation $\mathrm{htt}$ aggregates. ${ }^{43,58} \mathrm{Htt}$ with bonafide phosphorylation at $\mathrm{S} 13$ and S16 aggregated similarly to htt-constructs containing phosphomimetic mutations at these same sites. ${ }^{43,80,92}$ Therefore, it is demonstrated that serine phosphomimetic mutations are effective mimics for probing the impact of htt phosphorylation on aggregation.

\subsection{Significance of Lipid Interactions with Huntingtin Protein}

In amyloid-based neurodegenerative diseases like HD, membrane perturbation and permeabilization is a commonly proposed toxic mechanism. ${ }^{26,96}$ Cellular membrane are predominately comprised of lipids, which are comprised of a hydrophilic head and hydrophobic tail. Lipids readily self-assemble to form bilayers in aqueous solution. $\mathrm{Htt}$ itself is highly associated with a variety of membranes within cells, and localization to these membranes is a prominent feature in many normal functions attributed to htt. $55,87,91$ Several membrane related functions in which the lipid-binding properties of Nt17 may play a role, include cholesterol and energy homeostasis, ${ }^{97}$ cellular adhesion, ${ }^{98}$ motility, ${ }^{99,100}$ and molecular scaffolding. ${ }^{101} \mathrm{Htt}$ functions in the transport of lipid vesicles (including endocytic, synaptic or lysosomal), especially along microtubules. ${ }^{102-104}$ 


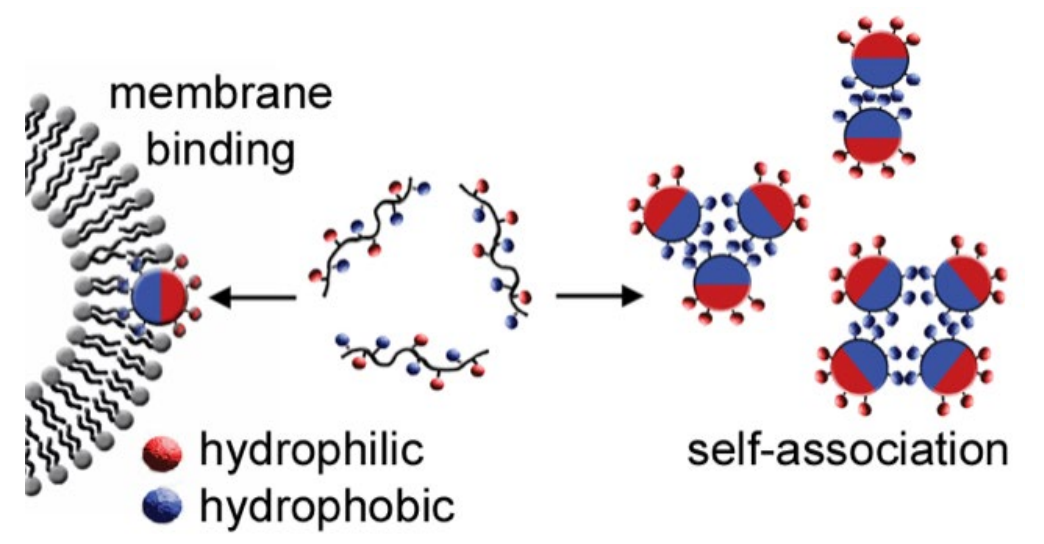

Figure 1.6: Mechanism of membrane binding interactions of htt. A key function of the AH of Nt17 is the ability to sense membranes. Nt17 is crucial for the mechanism of lipid membrane association of htt in that it forms two faces, hydrophobic and hydrophilic, in the presence of binding partners. These allow for self-association in early stages of aggregation, or the ability to preferentially bind to lipid membranes.

Nt17 also facilitates the trafficking of htt-exon1 to membranes associated with membranous organelles including ER, mitochondria, Golgi and autophagic vacuoles. ${ }^{105,106}$ Membrane association is also important for htt localization to subcellular components, such as the nucleus and plasma membrane. ${ }^{101}$ The ability of the $\mathrm{AH}$ structural qualities of Nt17 to engage membrane curvature sensing demonstrates a mechanistic role in these cellular functions. ${ }^{29}$ Specifically, htt trafficking between the nucleus and cytoplasm occurs through Nt17-regulated reversible association with several membranous organelles. ${ }^{105}$

With regard to toxicity, htt aggregates alter membrane structure and stability. ${ }^{45,48,105,107-109}$ Large unilamellar vesicles (LUV) that interact with htt-exon1 fragments enhance fibril formation in vitro. ${ }^{96}$ Mitochondrial and vesicular trafficking are impaired in htt knock-out mice, ${ }^{110,111} \mathrm{Htt}$ interacts with acidic phospholipids ${ }^{101}$ which could be instrumental in nucleating aggregation. Cumulative evidence suggests that a fundamental component of HD pathology is the consequence of toxic loss-of function, 
theoretically due to the disturbance of interactions between membranes and htt. $14,17,112,113 \mathrm{Htt}$ knockout mice display amplified apoptosis and premature embryonic lethality, ${ }^{17,112}$ and mice that are heterozygous for the htt knockout show significant neuronal degeneration, ${ }^{17,18}$ signifying that htt is a necessary protein for normal function. Membranes may represent direct targets of toxic htt aggregates. Htt-exon1 disturbs the structural integrity of lipid bilayers in a polyQ-dependent manner. ${ }^{114,115}$ Many aggregate species are localized to damaged membranes within cells, ${ }^{14,96,116,117}$ such as fibrils distorting ER morphology and dynamics in $\mathrm{HD},{ }^{117}$ and membranous structures are incorporated into htt inclusions. ${ }^{116} \mathrm{Htt}-\mathrm{exon} 1$ oligomers bind to and disrupts membranes consisting of brain lipids, and these interactions were correlated to membrane leakiness and neuronal toxicity. ${ }^{96}$

Altering the lipid composition of membranes plays an important role in $\mathrm{htt}$ aggregation. ${ }^{65}$ Lipid properties, including size, charge and degrees of saturation result in a broad array of physicochemical membrane features. Since physicochemical properties of a membrane system are governed by membrane composition, ${ }^{118}$ this explains why adjustments to membrane composition impact the interaction between htt and lipid surfaces. For example, the size of the lipid headgroup is key for developing the favored curvature of a bilayer system, ${ }^{119}$ while the size and saturation of the lipid tail impact features such as packing and fluidity. ${ }^{120}$ Amplified membrane fluidity results in a greater tendency for the occurrence of membrane defects, ${ }^{121}$ which ties into the defect detecting properties of the Nt17 domain. ${ }^{27}$ Structural analysis by solid state NMR demonstrates that Nt17 undergoes a structural transition from random-coil in solution to an $\alpha$-helical structure upon binding lipid bilayers. ${ }^{28,101}$ In addition, htt monomers disrupt 
the physical packing of POPC bilayers in a way that potentially favors intermolecular Nt17 association. ${ }^{63} \mathrm{PC} / \mathrm{PS}$ vesicles catalyze htt aggregation by promoting specific orientation within Nt17 on the membrane and increasing the local htt concentration on the surface. ${ }^{65}$ This effect is dependent on polyQ length and peptide to lipid ratios. ${ }^{122}$ Comparatively, the presence of total brain lipid extract (TBLE) diminishes fibrillization of htt. ${ }^{66,123}$ This supports the notion that lipid composition is a factor in the effect of a membrane on htt aggregation.

Nt17 plays an essential role in the ability of htt to bind membranes. While synthetic polyQ peptides lacking Nt17 did not appreciably interact with model lipid membranes, lipid membranes stabilized discrete oligomers of a Nt17Q 35 peptide, and a $\mathrm{Nt} 17 \mathrm{Q}_{35} \mathrm{P}_{10}$ peptide formed a variety of htt aggregates that readily damaged bilayers. ${ }^{26}$ Computational studies further back the vital role of $\mathrm{Nt} 17 \mathrm{in} \mathrm{htt} /$ lipid interactions and deliver evidence that the presence of both Nt17 and polyP flanking regions enhance the interaction of htt with bilayers. ${ }^{124}$ The supportive effect of Nt17 and the polyP domain in disrupting membranes may be principally significant considering interactions between these two domains in a cellular environment are altered when the polyQ domain is expanded beyond the threshold associated with HD. ${ }^{125}$ Induced mechanical changes in the membrane due to the buildup of monomeric htt and prefibrillar aggregates engaging directly on the surface disrupt bilayer integrity and could serve a role in htt-related toxic mechanisms. ${ }^{115,126}$ As the previously discussed PTMs are known to be involved in htt translocating to specific organelles ${ }^{105,127}$ and should be considered in regulating the interaction of htt with lipid membranes mediated by Nt17, they likely also impact the aggregation of htt in the presence of lipid membranes. 


\subsection{Dissertation Rationale}

Due to the importance of $\mathrm{Nt} 17$ in the interactions with membranous organelles and regulating aggregation, the roles that these interactions potentially play in $\mathrm{HD}$ pathology are crucial, yet unknown. Surfaces, particularly lipid surfaces associated with membranous organelles, have influences related with their interfaces. Such influences include electrostatic interactions, and the electrostatics between surfaces and htt have a huge role.

In Chapter 2, we explored combinations of changing the membrane electrostatics and changing the Nt17 electrostatics by mimicking a known post translational modification (PTM). Certain PTMs within Nt17 are known to modify HD pathology, and due to Nt17's inclination to interact with lipid membranes, the influence of these mutations were explored with various model lipid membranes. Specifically, phosphorylation at $\mathrm{T} 3, \mathrm{~S} 13$, and/or S16 improves phenotype and delays fibrilization in HD models. The impact of introducing phosphomimetic mutations (T3D, S13D and S16D) into htt-exon1 changed htt's interaction with and aggregation in the presence of lipids, reliant on the lipid system. Lipid systems included total brain lipid extract (TBLE), POPC and POPG, and were chosen to mimic a complex neutral lipid system, a neutral zwitterionic model lipid system, and a negatively charged lipid system.

Given that aggregation happens in a much more complicated environment, specifically in the highly crowded cytosol, the effects of macromolecular crowders present in the cytosol were examined with htt. In Chapter 3, changes were explored in the interfaces of membranes through the addition of various macromolecular crowders Dextran, Ficoll and PEG, with htt. This poses the question, if we crowd the liquid 
surface, i.e. the liquid portion of the solid/liquid interface, how does that impact the interactions at surfaces? The differences of crowding interactions with htt in a solid/liquid interface, represented by in situ experiments on mica, were compared to crowding a gel-like/liquid interface represented by in situ experiments on TBLE bilayers.

In addition, we adapted many of these assays and experiments used in the laboratory into new, upper level, undergraduate biochemistry laboratory experiments for students presented in an appendix. In short, over a six-week time span, students are immersed in a graduate level setting and perform an experiment with minimal guidance from prep work to completion. Students are asked to present their findings in a conference setting, with the final report written in the ACS journal Biochemistry format. Moving away from the traditional "cookbook" format of undergraduate laboratories, this experiment was well received as an addition to the undergraduate biochemistry curriculum.

\subsection{References}

(1) Irvine, G. B., El-Agnaf, O. M., Shankar, G. M., and Walsh, D. M. (2008) Protein Aggregation in the Brain: The Molecular Basis for Alzheimer's and Parkinson's Diseases. Mol Med 14, 451-464.

(2) Cook, C., Stetler, C., and Petrucelli, L. (2012) Disruption of Protein Quality Control in Parkinson's Disease. Cold Spring Harb Perspect Med 2.

(3) Arrasate, M., and Finkbeiner, S. (2012) Protein aggregates in Huntington's disease. Exp Neurol 238, 1-11.

(4) McGowan, D. P., van Roon-Mom, W., Holloway, H., Bates, G. P., Mangiarini, L., Cooper, G. J. S., Faull, R. L. M., and Snell, R. G. (2000) Amyloid-like inclusions in Huntington's disease. Neuroscience 100, 677-680.

(5) Kumar, J., Eraña, H., López-Martínez, E., Claes, N., Martín, V. F., Solís, D. M., Bals, S., Cortajarena, A. L., Castilla, J., and Liz-Marzán, L. M. (2018) Detection of amyloid fibrils in Parkinson's disease using plasmonic chirality. Proc Natl Acad Sci USA 115, 3225-3230.

(6) He, Z., Guo, J. L., McBride, J. D., Narasimhan, S., Kim, H., Changolkar, L., Zhang, B., Gathagan, R. J., Yue, C., Dengler, C., Stieber, A., Nitla, M., Coulter, D. A., Abel, T., Brunden, K. R., Trojanowski, J. Q., and Lee, V. M.-Y. (2018) Amyloid- $\beta$ plaques 
enhance Alzheimer's brain tau-seeded pathologies by facilitating neuritic plaque tau aggregation. Nat Med 24, 29-38.

(7) Nelson, R., Sawaya, M. R., Balbirnie, M., Madsen, A. Ø., Riekel, C., Grothe, R., and Eisenberg, D. (2005) Structure of the cross $-\beta$ spine of amyloid-like fibrils. Nature 435 , 773-778.

(8) Wetzel, R. (2012) Physical chemistry of polyglutamine: Intriguing tales of a monotonous sequence. J Mol Biol 421, 466-490.

(9) Xi, E., and Patel, A. J. (2016) The hydrophobic effect, and fluctuations: The long and the short of it. Proc Natl Acad Sci USA 113, 4549-4551.

(10) Onuchic, J. N., Luthey-Schulten, Z., and Wolynes, P. G. (1997) THEORY OF PROTEIN FOLDING: The Energy Landscape Perspective. Annu Rev Phys Chem 48, 545-600.

(11) Onuchic, J. N., Wolynes, P. G., Luthey-Schulten, Z., and Socci, N. D. (1995)

Toward an outline of the topography of a realistic protein-folding funnel. Proc Natl Acad Sci USA 92, 3626-3630.

(12) Bryngelson, J. D., Onuchic, J. N., Socci, N. D., and Wolynes, P. G. (1995) Funnels, pathways, and the energy landscape of protein folding: A synthesis. Proteins 21, 167195.

(13) Leopold, P. E., Montal, M., and Onuchic, J. N. (1992) Protein folding funnels: a kinetic approach to the sequence-structure relationship. Proc Natl Acad Sci USA 89, 8721-8725.

(14) Adegbuyiro, A., Sedighi, F., Pilkington, A. W., Groover, S., and Legleiter, J. (2017) Proteins Containing Expanded Polyglutamine Tracts and Neurodegenerative Disease. Biochemistry 56, 1199-1217.

(15) Shorter, J., and Lindquist, S. (2005) Prions as adaptive conduits of memory and inheritance. Nat Rev Genet 6, 435-450.

(16) Jahn, T. R., and Radford, S. E. (2008) Folding versus aggregation: Polypeptide conformations on competing pathways. Arch Biochem Biophys 469, 100-117.

(17) Nasir, J., Floresco, S. B., O'Kusky, J. R., Diewert, V. M., Richman, J. M., Zeisler, J., Borowski, A., Marth, J. D., Phillips, A. G., and Hayden, M. R. (1995) Targeted disruption of the Huntington's disease gene results in embryonic lethality and behavioral and morphological changes in heterozygotes. Cell 81, 811-823.

(18) O'Kusky, J. R., Nasir, J., Cicchetti, F., Parent, A., and Hayden, M. R. (1999) Neuronal degeneration in the basal ganglia and loss of pallido-subthalamic synapses in mice with targeted disruption of the Huntington's disease gene. Brain Res 818, 468479.

(19) Macdonald, M. (1993) A novel gene containing a trinucleotide repeat that is expanded and unstable on Huntington's disease chromosomes. Cell 72, 971-983. (20) Burke, K. A., Godbey, J., and Legleiter, J. (2011) Assessing mutant huntingtin fragment and polyglutamine aggregation by atomic force microscopy. Methods 53, 275284.

(21) Poirier, M. A., Li, H., Macosko, J., Cai, S., Amzel, M., and Ross, C. A. (2002) Huntingtin Spheroids and Protofibrils as Precursors in Polyglutamine Fibrilization. J Biol Chem 277, 41032-41037.

(22) Snell, R. G., MacMillan, J. C., Cheadle, J. P., Fenton, I., Lazarou, L. P., Davies, P., MacDonald, M. E., Gusella, J. F., Harper, P. S., and Shaw, D. J. (1993) Relationship 
between trinucleotide repeat expansion and phenotypic variation in Huntington's disease. Nat Genet 4, 393-397.

(23) Tobin, A. J., and Signer, E. R. (2000) Huntington's disease: the challenge for cell biologists. Trend Cell Biol 10, 531-536.

(24) Andrew, S. E., Paul Goldberg, Y., Kremer, B., Telenius, H., Theilmann, J., Adam, S., Starr, E., Squitieri, F., Lin, B., Kalchman, M. A., Graham, R. K., and Hayden, M. R. (1993) The relationship between trinucleotide (CAG) repeat length and clinical features of Huntington's disease. Nat Genet 4, 398-403.

(25) Ross, C. A., Poirier, M. A., Wanker, E. E., and Amzel, M. (2003) Polyglutamine fibrillogenesis: The pathway unfolds. Proc Natl Acad Sci USA 100, 1-3.

(26) Burke, K. A., Kauffman, K. J., Umbaugh, C. S., Frey, S. L., and Legleiter, J. (2013) The Interaction of Polyglutamine Peptides with Lipid Membranes Is Regulated by Flanking Sequences Associated with Huntingtin. J Biol Chem 288, 14993-15005. (27) Arndt, J. R., Chaibva, M., and Legleiter, J. (2015) The emerging role of the first 17 amino acids of huntingtin in Huntington's disease. Biomol Concepts 6, 33-46.

(28) Michalek, M., Salnikov, E. S., and Bechinger, B. (2013) Structure and Topology of the Huntingtin 1-17 Membrane Anchor by a Combined Solution and Solid-State NMR Approach. Biophys J 105, 699-710.

(29) Chaibva, M., Burke, K. A., and Legleiter, J. (2014) Curvature Enhances Binding and Aggregation of Huntingtin at Lipid Membranes. Biochemistry 53, 2355-2365.

(30) Williamson, T. E., Vitalis, A., Crick, S. L., and Pappu, R. V. (2010) Modulation of polyglutamine conformations and dimer formation by the $\mathrm{N}$-terminus of Huntingtin. $\mathrm{J} \mathrm{Mol}$ Biol 396, 1295-1309.

(31) Arndt, J. R., Chaibva, M., Beasley, M., Kiani Karanji, A., Ghassabi Kondalaji, S., Khakinejad, M., Sarver, O., Legleiter, J., and Valentine, S. J. (2020) Nucleation Inhibition of Huntingtin Protein (htt) by Polyproline PPII Helices: A Potential Interaction with the N-Terminal $\alpha$-Helical Region of Htt. Biochemistry 59, 436-449.

(32) Landles, C., Sathasivam, K., Weiss, A., Woodman, B., Moffitt, H., Finkbeiner, S., Sun, B., Gafni, J., Ellerby, L. M., Trottier, Y., Richards, W. G., Osmand, A., Paganetti, P., and Bates, G. P. (2010) Proteolysis of Mutant Huntingtin Produces an Exon 1 Fragment That Accumulates as an Aggregated Protein in Neuronal Nuclei in Huntington Disease. J Biol Chem 285, 8808-8823.

(33) Davies, S. W., Turmaine, M., Cozens, B. A., Difiglia, M., Sharp, A. H., Ross, C. A., Scherzinger, E., Wanker, E. E., Mangiarini, L., and Bates, G. P. (1997) Formation of Neuronal Intranuclear Inclusions Underlies the Neurological Dysfunction in Mice Transgenic for the HD Mutation. Cell 90, 537-548.

(34) Sathasivam, K., Lane, A., Legleiter, J., Warley, A., Woodman, B., Finkbeiner, S., Paganetti, P., Muchowski, P. J., Wilson, S., and Bates, G. P. (2010) Identical oligomeric and fibrillar structures captured from the brains of R6/2 and knock-in mouse models of Huntington's disease. Hum Mol Genet 19, 65-78.

(35) Kim, Y. J., Yi, Y., Sapp, E., Wang, Y., Cuiffo, B., Kegel, K. B., Qin, Z.-H., Aronin, N., and DiFiglia, M. (2001) Caspase 3-cleaved N-terminal fragments of wild-type and mutant huntingtin are present in normal and Huntington's disease brains, associate with membranes, and undergo calpain-dependent proteolysis. Proc Natl Acad Sci USA 98, 12784-12789. 
(36) Hoffner, G., Island, M.-L., and Djian, P. (2005) Purification of neuronal inclusions of patients with Huntington's disease reveals a broad range of N-terminal fragments of expanded huntingtin and insoluble polymers. J Neurochem 95, 125-136.

(37) Cooper, J. (1998) Truncated N-terminal fragments of huntingtin with expanded glutamine repeats form nuclear and cytoplasmic aggregates in cell culture. Hum $\mathrm{Mol}$ Genet 7, 783-790.

(38) Yang, H., Yang, S., Jing, L., Huang, L., Chen, L., Zhao, X., Yang, W., Pan, Y., Yin, P., Qin, Z. S., Li, S., and Li, X.-J. (2020) Truncation of mutant huntingtin in knock-in mice demonstrates exon1 huntingtin is a key pathogenic form. Nat Commun 11, 2582. (39) Jones, A. L. (1999) The localization and interactions of huntingtin. Philos Trans $R$ Soc Lond B Biol Sci 354, 1021-1027.

(40) Arndt, J. R., Kondalaji, S. G., Maurer, M. M., Parker, A., Legleiter, J., and Valentine, S. J. (2015) Huntingtin N-terminal monomeric and multimeric structures destabilized by covalent modification of heteroatomic residues. Biochemistry $54,4285-$ 4296.

(41) Jayaraman, M., Kodali, R., Sahoo, B., Thakur, A. K., Mayasundari, A., Mishra, R., Peterson, C. B., and Wetzel, R. (2012) Slow Amyloid Nucleation via a-Helix-Rich Oligomeric Intermediates in Short Polyglutamine-Containing Huntingtin Fragments. $J$ Mol Biol 415, 881-899.

(42) Legleiter, J., Mitchell, E., Lotz, G. P., Sapp, E., Ng, C., DiFiglia, M., Thompson, L. M., and Muchowski, P. J. (2010) Mutant Huntingtin Fragments Form Oligomers in a Polyglutamine Length-dependent Manner in Vitro and in Vivo. J Biol Chem 285, 1477714790.

(43) Gu, X., Greiner, E. R., Mishra, R., Kodali, R., Osmand, A., Finkbeiner, S., Steffan, J. S., Thompson, L. M., Wetzel, R., and Yang, X. W. (2009) Serines 13 and 16 Are Critical Determinants of Full-length Human Mutant Huntingtin-Induced Disease Pathogenesis in HD Mice. Neuron 64, 828-840.

(44) Kar, K., Jayaraman, M., Sahoo, B., Kodali, R., and Wetzel, R. (2011) Critical nucleus size for disease-related polyglutamine aggregation is repeat length dependent. Nat Struct Mol Biol 18, 328-336.

(45) Mannini, B., Cascella, R., Zampagni, M., van Waarde-Verhagen, M., Meehan, S., Roodveldt, C., Campioni, S., Boninsegna, M., Penco, A., Relini, A., Kampinga, H. H., Dobson, C. M., Wilson, M. R., Cecchi, C., and Chiti, F. (2012) Molecular mechanisms used by chaperones to reduce the toxicity of aberrant protein oligomers. Proc Natl Acad Sci USA 109, 12479-12484.

(46) Xue, W.-F., Homans, S. W., and Radford, S. E. (2008) Systematic analysis of nucleation-dependent polymerization reveals new insights into the mechanism of amyloid self-assembly. Proc Natl Acad Sci USA 105, 8926-8931.

(47) Saudou, F., Finkbeiner, S., Devys, D., and Greenberg, M. E. (1998) Huntingtin Acts in the Nucleus to Induce Apoptosis but Death Does Not Correlate with the Formation of Intranuclear Inclusions. Cell 95, 55-66.

(48) Chen, S. et al. Polyglutamine Aggregation Behavior in vitro Supports a Recruitment Mechanism of Cytotoxicity. J Mol Biol 331 (1), 173-82.

(49) Nagai, Y., Inui, T., Popiel, H. A., Fujikake, N., Hasegawa, K., Urade, Y., Goto, Y., Naiki, H., and Toda, T. (2007) A toxic monomeric conformer of the polyglutamine protein. Nat Struct Molec Biol 14, 332-340. 
(50) Thakur, A. K., Jayaraman, M., Mishra, R., Thakur, M., Chellgren, V. M., Byeon, I.J., Anjum, D. H., Kodali, R., Creamer, T. P., Conway, J. F., M.Gronenborn, A., and Wetzel, R. (2009) Polyglutamine disruption of the huntingtin exon1 N-terminus triggers a complex aggregation mechanism. Nat Struct Mol Biol 16, 380-389.

(51) Mishra, R., Hoop, C. L., Kodali, R., Sahoo, B., van der Wel, P. C. A., and Wetzel, R. (2012) Serine phosphorylation suppresses huntingtin amyloid accumulation by altering protein aggregation properties. J Mol Biol 424, 1-14.

(52) Sahoo, B., Arduini, I., Drombosky, K. W., Kodali, R., Sanders, L. H., Greenamyre, J. T., and Wetzel, R. (2016) Folding Landscape of Mutant Huntingtin Exon1: Diffusible Multimers, Oligomers and Fibrils, and No Detectable Monomer. PLOS ONE (Borchelt, D. R., Ed.) 11, e0155747.

(53) Sahoo, B., Singer, D., Kodali, R., Zuchner, T., and Wetzel, R. (2014) Aggregation Behavior of Chemically Synthesized, Full-Length Huntingtin Exon1. Biochemistry 53, 3897-3907.

(54) Drin, G., and Antonny, B. (2010) Amphipathic helices and membrane curvature. FEBS Letters 584, 1840-1847.

(55) Kegel-Gleason, K. B. (2013) Huntingtin Interactions with Membrane Phospholipids: Strategic Targets for Therapeutic Intervention? JHD 2, 239-250.

(56) Yamauchi, Y., Deguchi, N., Takagi, C., Tanaka, M., Dhanasekaran, P., Nakano, M., Handa, T., Phillips, M. C., Lund-Katz, S., and Saito, H. (2008) Role of the N- and Cterminal Domains in Binding of Apolipoprotein E Isoforms to Heparan Sulfate and Dermatan Sulfate: A Surface Plasmon Resonance Study. Biochemistry 47, 6702-6710. (57) Gouttenoire, J., Roingeard, P., Penin, F., and Moradpour, D. (2010) Amphipathic aHelix AH2 Is a Major Determinant for the Oligomerization of Hepatitis C Virus Nonstructural Protein 4B. J Virol 84, 12529-12537.

(58) Mishra, R., Jayaraman, M., Roland, B. P., Landrum, E., Fullam, T., Kodali, R., Thakur, A. K., Arduini, I., and Wetzel, R. (2012) Inhibiting nucleation of amyloid structure in a huntingtin fragment by targeting a-helix rich oligomeric intermediates. $J$ Mol Biol 415, 900-917.

(59) Lajoie, P., and Snapp, E. L. (2010) Formation and Toxicity of Soluble Polyglutamine Oligomers in Living Cells. PLoS ONE (Chirico, G., Ed.) 5, e15245. (60) Nucifora, L. G., Burke, K. A., Feng, X., Arbez, N., Zhu, S., Miller, J., Yang, G., Ratovitski, T., Delannoy, M., Muchowski, P. J., Finkbeiner, S., Legleiter, J., Ross, C. A., and Poirier, M. A. (2012) Identification of Novel Potentially Toxic Oligomers Formed in Vitro from Mammalian-derived Expanded huntingtin Exon-1 Protein. J Biol Chem 287, 16017-16028.

(61) Kim, Y. E., Hosp, F., Frottin, F., Ge, H., Mann, M., Hayer-Hartl, M., and Hartl, F. U. (2016) Soluble Oligomers of PolyQ-Expanded Huntingtin Target a Multiplicity of Key Cellular Factors. Mol Cell 63, 951-964.

(62) Pieri, L., Madiona, K., Bousset, L., and Melki, R. (2012) Fibrillar a-Synuclein and Huntingtin Exon 1 Assemblies Are Toxic to the Cells. Biophys J 102, 2894-2905.

(63) Côté, S., Binette, V., Salnikov, E. S., Bechinger, B., and Mousseau, N. (2015) Probing the Huntingtin 1-17 Membrane Anchor on a Phospholipid Bilayer by Using AllAtom Simulations. Biophys J 108, 1187-1198. 
(64) Côté, S., Wei, G., and Mousseau, N. (2014) Atomistic mechanisms of huntingtin Nterminal fragment insertion on a phospholipid bilayer revealed by molecular dynamics simulations. Proteins 82, 1409-1427.

(65) Pandey, N. K., Isas, J. M., Rawat, A., Lee, R. V., Langen, J., Pandey, P., and Langen, $R$. (2018) The 17-residue-long $\mathrm{N}$ terminus in huntingtin controls stepwise aggregation in solution and on membranes via different mechanisms. J Biol Chem 293, 2597-2605.

(66) Chaibva, M., Gao, X., Jain, P., Campbell, W. A., Frey, S. L., and Legleiter, J. (2018) Sphingomyelin and GM1 Influence Huntingtin Binding to, Disruption of, and Aggregation on Lipid Membranes. ACS Omega 3, 273-285.

(67) Bigay, J., Casella, J.-F., Drin, G., Mesmin, B., and Antonny, B. (2005) ArfGAP1 responds to membrane curvature through the folding of a lipid packing sensor motif. EMBO J 24, 2244-2253.

(68) Mesmin, B., Drin, G., Levi, S., Rawet, M., Cassel, D., Bigay, J., and Antonny, B. (2007) Two Lipid-Packing Sensor Motifs Contribute to the Sensitivity of ArfGAP1 to Membrane Curvature. Biochemistry 46, 1779-1790.

(69) Steffan, J. S., Agrawal, N., Pallos, J., Rockabrand, E., Trotman, L. C., Slepko, N., Illes, K., Lukacsovich, T., Zhu, Y.-Z., Cattaneo, E., Pandolfi, P. P., Thompson, L. M., and Marsh, J. L. (2004) SUMO Modification of Huntingtin and Huntington's Disease Pathology. Science 304, 100-104.

(70) Pennuto, M., Palazzolo, I., and Poletti, A. (2009) Post-translational modifications of expanded polyglutamine proteins: impact on neurotoxicity. Hum Mol Genet 18, R40R47.

(71) Ehrnhoefer, D. E., Sutton, L., and Hayden, M. R. (2011) Small changes, big impact: Posttranslational modifications and function of huntingtin in Huntington disease.

Neuroscientist 17, 475-492.

(72) Cong, X., Held, J. M., DeGiacomo, F., Bonner, A., Chen, J. M., Schilling, B., Czerwieniec, G. A., Gibson, B. W., and Ellerby, L. M. (2011) Mass Spectrometric Identification of Novel Lysine Acetylation Sites in Huntingtin. Mol Cell Proteomics 10. (73) Jeong, H., Then, F., Melia, T. J., Mazzulli, J. R., Cui, L., Savas, J. N., Voisine, C., Paganetti, P., Tanese, N., Hart, A. C., Yamamoto, A., and Krainc, D. (2009) Acetylation Targets Mutant Huntingtin to Autophagosomes for Degradation. Cell 137, 60-72.

(74) Ceccon, A., Schmidt, T., Tugarinov, V., Kotler, S. A., Schwieters, C. D., and Clore, G. M. (2018) Interaction of Huntingtin Exon-1 Peptides with Lipid-Based Micellar Nanoparticles Probed by Solution NMR and Q-Band Pulsed EPR. J Am Chem Soc 140, 6199-6202.

(75) O’Rourke, J. G., Gareau, J. R., Ochaba, J., Song, W., Raskó, T., Reverter, D., Lee, J., Monteys, A. M., Pallos, J., Mee, L., Vashishtha, M., Apostol, B. L., Nicholson, T. P., Illes, K., Zhu, Y.-Z., Dasso, M., Bates, G. P., Difiglia, M., Davidson, B., Wanker, E. E., Marsh, J. L., Lima, C. D., Steffan, J. S., and Thompson, L. M. (2013) SUMO-2 and PIAS1 Modulate Insoluble Mutant Huntingtin Protein Accumulation. Cell Rep 4, 362375.

(76) Sedighi, F., Adegbuyiro, A., and Legleiter, J. (2020) SUMOylation Prevents Huntingtin Fibrillization and Localization onto Lipid Membranes. ACS Chem Neurosci 11, 328-343. 
(77) Kalchman, M. A., Graham, R. K., Xia, G., Koide, H. B., Hodgson, J. G., Graham, K. C., Goldberg, Y. P., Gietz, R. D., Pickart, C. M., and Hayden, M. R. (1996) Huntingtin Is Ubiquitinated and Interacts with a Specific Ubiquitin-conjugating Enzyme. J Biol Chem 271, 19385-19394.

(78) Aiken, C. T., Steffan, J. S., Guerrero, C. M., Khashwji, H., Lukacsovich, T., Simmons, D., Purcell, J. M., Menhaji, K., Zhu, Y.-Z., Green, K., LaFerla, F., Huang, L., Thompson, L. M., and Marsh, J. L. (2009) Phosphorylation of Threonine 3. J Biol Chem 284, 29427-29436.

(79) Di Pardo, A., Maglione, V., Alpaugh, M., Horkey, M., Atwal, R. S., Sassone, J., Ciammola, A., Steffan, J. S., Fouad, K., Truant, R., and Sipione, S. (2012) Ganglioside GM1 induces phosphorylation of mutant huntingtin and restores normal motor behavior in Huntington disease mice. Proc Natl Acad Sci USA 109, 3528-3533.

(80) Thompson, L. M., Aiken, C. T., Kaltenbach, L. S., Agrawal, N., Illes, K., Khoshnan, A., Martinez-Vincente, M., Arrasate, M., O'Rourke, J. G., Khashwji, H., Lukacsovich, T., Zhu, Y.-Z., Lau, A. L., Massey, A., Hayden, M. R., Zeitlin, S. O., Finkbeiner, S., Green, K. N., LaFerla, F. M., Bates, G., Huang, L., Patterson, P. H., Lo, D. C., Cuervo, A. M., Marsh, J. L., and Steffan, J. S. (2009) IKK phosphorylates Huntingtin and targets it for degradation by the proteasome and lysosome. J Cell Biol 187, 1083-1099.

(81) DeGuire, S. M., Ruggeri, F. S., Fares, M.-B., Chiki, A., Cendrowska, U., Dietler, G., and Lashuel, H. A. (2018) N-terminal Huntingtin (Htt) phosphorylation is a molecular switch regulating $\mathrm{Htt}$ aggregation, helical conformation, internalization, and nuclear targeting. J Biol Chem 293, 18540-18558.

(82) Yanai, A., Huang, K., Kang, R., Singaraja, R. R., Arstikaitis, P., Gan, L., Orban, P. C., Mullard, A., Cowan, C. M., Raymond, L. A., Drisdel, R. C., Green, W. N., Ravikumar, B., Rubinsztein, D. C., El-Husseini, A., and Hayden, M. R. (2006) Palmitoylation of huntingtin by HIP14 is essential for its trafficking and function. Nat Neurosci 9, 824-831. (83) Kahlem, P., Green, H., and Djian, P. (1998) Transglutaminase Action Imitates Huntington's Disease: Selective Polymerization of Huntingtin Containing Expanded Polyglutamine. Mol Cell 1, 595-601.

(84) Dorval, V., and Fraser, P. E. (2007) SUMO on the road to neurodegeneration. BBA - Mol Cell Res 1773, 694-706.

(85) Borrell-Pagès, M., Zala, D., Humbert, S., and Saudou, F. (2006) Huntington's disease: from huntingtin function and dysfunction to therapeutic strategies. Cell Mol Life Sci 63, 2642-2660.

(86) Díaz-Hernández, M., Valera, A. G., Morán, M. A., Gómez-Ramos, P., AlvarezCastelao, B., Castaño, J. G., Hernández, F., and Lucas, J. J. (2006) Inhibition of $26 S$ proteasome activity by huntingtin filaments but not inclusion bodies isolated from mouse and human brain. $J$ Neurochem 98, 1585-1596.

(87) Chaibva, M., Arndt, J. R., Valentine, S. J., and Legleiter, J. (2015) Acetylation Regulates the Interaction of Huntingtin with Lipid Membranes: Implications for Huntington Disease. Biophys J 108, 254a.

(88) Arndt, J. R., Brown, R. J., Burke, K. A., Legleiter, J., and Valentine, S. J. (2015) Lysine residues in the $\mathrm{N}$-terminal huntingtin amphipathic a-helix play a key role in peptide aggregation. J Mass Spec 50, 117-126.

(89) Subramaniam, S., Sixt, K. M., Barrow, R., and Snyder, S. H. (2009) Rhes, a Striatal Specific Protein, Mediates Mutant-Huntingtin Cytotoxicity. Science 324, 1327-1330. 
(90) Jana, N. R., Dikshit, P., Goswami, A., Kotliarova, S., Murata, S., Tanaka, K., and Nukina, N. (2005) Co-chaperone CHIP Associates with Expanded Polyglutamine Protein and Promotes Their Degradation by Proteasomes. J Biol Chem 280, 1163511640.

(91) DiFiglia, M., Sapp, E., Chase, K. O., Davies, S. W., Bates, G. P., Vonsattel, J. P., and Aronin, N. (1997) Aggregation of Huntingtin in Neuronal Intranuclear Inclusions and Dystrophic Neurites in Brain. Science 277, 1990-1993.

(92) Atwal, R. S., Desmond, C. R., Caron, N., Maiuri, T., Xia, J., Sipione, S., and Truant, R. (2011) Kinase inhibitors modulate huntingtin cell localization and toxicity. Nat Chem Biol 7, 453-460.

(93) Watkin, E. E., Arbez, N., Waldron-Roby, E., O'Meally, R., Ratovitski, T., Cole, R. N., and Ross, C. A. (2014) Phosphorylation of Mutant Huntingtin at Serine 116 Modulates Neuronal Toxicity. PLoS One 9.

(94) Chiki, A., DeGuire, S. M., Ruggeri, F. S., Sanfelice, D., Ansaloni, A., Wang, Z.-M., Cendrowska, U., Burai, R., Vieweg, S., Pastore, A., Dietler, G., and Lashuel, H. A. (2017) Mutant Exon1 Huntingtin Aggregation is Regulated by T3 PhosphorylationInduced Structural Changes and Crosstalk between T3 Phosphorylation and Acetylation at K6. Angew Chem Intl Ed 56, 5202-5207.

(95) Cariulo, C., Azzollini, L., Verani, M., Martufi, P., Boggio, R., Chiki, A., Deguire, S. M., Cherubini, M., Gines, S., Marsh, J. L., Conforti, P., Cattaneo, E., Santimone, I., Squitieri, F., Lashuel, H. A., Petricca, L., and Caricasole, A. (2017) Phosphorylation of huntingtin at residue T3 is decreased in Huntington's disease and modulates mutant huntingtin protein conformation. Proc Natl Acad Sci USA 114, E10809-E10818. (96) Suopanki, J., Götz, C., Lutsch, G., Schiller, J., Harjes, P., Herrmann, A., and Wanker, E. E. (2006) Interaction of huntingtin fragments with brain membranes - clues to early dysfunction in Huntington's disease. J Neurochem 96, 870-884.

(97) Jacobsen, J. C., Gregory, G. C., Woda, J. M., Thompson, M. N., Coser, K. R., Murthy, V., Kohane, I. S., Gusella, J. F., Seong, I. S., MacDonald, M. E., Shioda, T., and Lee, J.-M. (2011) HD CAG-correlated gene expression changes support a simple dominant gain of function. Hum Mol Genet 20, 2846-2860.

(98) Strehlow, A. N. T., Li, J. Z., and Myers, R. M. (2007) Wild-type huntingtin participates in protein trafficking between the Golgi and the extracellular space. Hum Mol Genet 16, 391-409.

(99) Ritch, J. J., Valencia, A., Alexander, J., Sapp, E., Gatune, L., Sangrey, G. R., Sinha, S., Scherber, C. M., Zeitlin, S., Sadri-Vakili, G., Irimia, D., DiFiglia, M., and Kegel, K. B. (2012) Multiple Phenotypes in Huntington Disease Mouse Neural Stem Cells. Mol Cell Neurosci 50, 70-81.

(100) Amarnath, S., Kawli, T., Mohanty, S., Srinivasan, N., and Nanjundiah, V. (2012) Pleiotropic Roles of a Ribosomal Protein in Dictyostelium discoideum. PLoS One 7. (101) Kegel, K. B., Sapp, E., Yoder, J., Cuiffo, B., Sobin, L., Kim, Y. J., Qin, Z.-H., Hayden, M. R., Aronin, N., Scott, D. L., Isenberg, G., Goldmann, W. H., and DiFiglia, M. (2005) Huntingtin Associates with Acidic Phospholipids at the Plasma Membrane. J Biol Chem 280, 36464-36473.

(102) Gunawardena, S., Her, L.-S., Brusch, R. G., Laymon, R. A., Niesman, I. R., Gordesky-Gold, B., Sintasath, L., Bonini, N. M., and Goldstein, L. S. B. (2003) 
Disruption of Axonal Transport by Loss of Huntingtin or Expression of Pathogenic PolyQ Proteins in Drosophila. Neuron 40(1), 25-40.

(103) Gauthier, L. R., Charrin, B. C., Borrell-Pagès, M., Dompierre, J. P., Rangone, H., Cordelières, F. P., De Mey, J., MacDonald, M. E., Leßmann, V., Humbert, S., and Saudou, F. (2004) Huntingtin Controls Neurotrophic Support and Survival of Neurons by Enhancing BDNF Vesicular Transport along Microtubules. Cell 118, 127-138.

(104) Pal, A., Severin, F., Lommer, B., Shevchenko, A., and Zerial, M. effector that regulates early endosome motility and is up regulated in Huntington's disease 14. (105) Atwal, R. S., Xia, J., Pinchev, D., Taylor, J., Epand, R. M., and Truant, R. (2007) Huntingtin has a membrane association signal that can modulate huntingtin aggregation, nuclear entry and toxicity. Hum Mol Genet 16, 2600-2615.

(106) Rockabrand, E., Slepko, N., Pantalone, A., Nukala, V. N., Kazantsev, A., Marsh, J. L., Sullivan, P. G., Steffan, J. S., Sensi, S. L., and Thompson, L. M. (2007) The first 17 amino acids of Huntingtin modulate its sub-cellular localization, aggregation and effects on calcium homeostasis. Hum Mol Genet 16, 61-77.

(107) Zaman, M., Ahmad, E., Qadeer, A., Rabbani, G., and Khan, R. H. (2014)

Nanoparticles in relation to peptide and protein aggregation. Int J Nanomedicine 9, 899912.

(108) Xi, W., Wang, X., Laue, T. M., and Denis, C. L. (2016) Multiple discrete soluble aggregates influence polyglutamine toxicity in a Huntington's disease model system. Sci Rep 6, 34916.

(109) Todd, T. W., and Lim, J. (2013) Aggregation formation in the polyglutamine diseases: Protection at a cost? Mol Cells 36, 185-194.

(110) Trushina, E., Dyer, R. B., Badger, J. D., Ure, D., Eide, L., Tran, D. D., Vrieze, B. T., Legendre-Guillemin, V., McPherson, P. S., Mandavilli, B. S., Van Houten, B., Zeitlin, S., McNiven, M., Aebersold, R., Hayden, M., Parisi, J. E., Seeberg, E., Dragatsis, I., Doyle, K., Bender, A., Chacko, C., and McMurray, C. T. (2004) Mutant Huntingtin Impairs Axonal Trafficking in Mammalian Neurons In Vivo and In Vitro. Mol Cell Biol 24, 8195-8209.

(111) Dragatsis, I., Levine, M. S., and Zeitlin, S. (2000) Inactivation of Hdh in the brain and testis results in progressive neurodegeneration and sterility in mice. Nat Genet 26, 300-306.

(112) Zeitlin, S., Liu, J.-P., Chapman, D. L., Papaioannou, V. E., and Efstratiadis, A. (1995) Increased apoptosis and early embryonic lethality in mice nullizygous for the Huntington's disease gene homologue. Nat Genet 11, 155-163.

(113) Cisbani, G., and Cicchetti, F. (2012) An in vitro perspective on the molecular mechanisms underlying mutant huntingtin protein toxicity. Cell Death Dis 3, e382-e382. (114) Kegel, K. B., Sapp, E., Alexander, J., Valencia, A., Reeves, P., Li, X., Masso, N., Sobin, L., Aronin, N., and DiFiglia, M. (2009) Polyglutamine expansion in huntingtin alters its interaction with phospholipids. J Neurochem 110, 1585-1597.

(115) Burke, K. A., Hensal, K. M., Umbaugh, C. S., Chaibva, M., and Legleiter, J. (2013) Huntingtin disrupts lipid bilayers in a polyQ-length dependent manner. BBA Biomembranes 1828, 1953-1961.

(116) Kegel, K. B., Kim, M., Sapp, E., Mclntyre, C., Castaño, J. G., Aronin, N., and DiFiglia, M. (2000) Huntingtin Expression Stimulates Endosomal-Lysosomal Activity, Endosome Tubulation, and Autophagy. J Neurosci 20, 7268-7278. 
(117) Guedes-Dias, P., and Holzbaur, E. L. F. (2017) Huntingtin Fibrils Poke Membranes. Cell 171, 32-33.

(118) Haque, Md. E., McIntosh, T. J., and Lentz, B. R. (2001) Influence of Lipid Composition on Physical Properties and PEG-Mediated Fusion of Curved and Uncurved Model Membrane Vesicles: "Nature's Own” Fusogenic Lipid Bilayer. Biochemistry 40, 4340-4348.

(119) Cooke, I. R., and Deserno, M. (2006) Coupling between Lipid Shape and Membrane Curvature. Biophys J 91, 487-495.

(120) Larsen, J. B., Kennard, C., Pedersen, S. L., Jensen, K. J., Uline, M. J., Hatzakis, N. S., and Stamou, D. (2017) Membrane Curvature and Lipid Composition Synergize To Regulate N-Ras Anchor Recruitment. Biophys J 113, 1269-1279.

(121) Bennett, W. F. D., and Tieleman, D. P. (2014) The Importance of Membrane Defects-Lessons from Simulations. Acc Chem Res 47, 2244-2251.

(122) Marquette, A., and Bechinger, B. (2020) Membrane interactions accelerate the self-aggregation of huntingtin exon 1 fragments in a polyglutamine length-dependent manner. preprint, Cold Spring Harbor Laboratory.

(123) Beasley, M., Stonebraker, A. R., Hasan, I., Kapp, K. L., Liang, B. J., Agarwal, G., Groover, S., Sedighi, F., and Legleiter, J. (2019) Lipid Membranes Influence the Ability of Small Molecules To Inhibit Huntingtin Fibrillization. Biochemistry 58, 4361-4373. (124) Michalek, M., Salnikov, E. S., Werten, S., and Bechinger, B. (2013) Membrane Interactions of the Amphipathic Amino Terminus of Huntingtin. Biochemistry 52, 847858.

(125) Caron, N. S., Desmond, C. R., Xia, J., and Truant, R. (2013) Polyglutamine domain flexibility mediates the proximity between flanking sequences in huntingtin. Proc Natl Acad Sci USA 110, 14610-14615.

(126) Burke, K. A., Yates, E. A., and Legleiter, J. (2013) Amyloid-Forming Proteins Alter the Local Mechanical Properties of Lipid Membranes. Biochemistry 52, 808-817. (127) Xia, J. (2003) Huntingtin contains a highly conserved nuclear export signal. Human Molecular Genetics 12, 1393-1403. 


\title{
2. Phosphomimetic Mutations Impact Huntingtin Aggregation in the Presence of a Variety of Lipid Systems
}

\begin{abstract}
Huntington's Disease (HD) is a neurodegenerative disorder caused by the abnormal expansion of a polyglutamine (polyQ) tract in the first exon of the htt protein (htt). PolyQ expansion triggers the aggregation of htt into a variety of structures, including oligomers and fibrils. This aggregation is impacted by the first $17 \mathrm{~N}$-terminal amino acids (Nt17) of htt that directly precedes the polyQ domain. Beyond impacting aggregation, Nt17 associates with lipid membranes by forming an amphipathic $\alpha$-helix. Post-translational modifications within Nt17 are known to modify HD pathology, and in particular, phosphorylation at T3, S13, and/or S16 retard fibrilization and ameliorates phenotype in HD models. Due to Nt17's propensity to interact with lipid membranes, the impact of introducing phosphomimetic mutations (T3D, S13D and S16D) into htt-exon1 on aggregation in the presence of a variety of model lipid membranes (total brain lipid extract, POPC, and POPG) was investigated. Phosphomimetic mutations altered htt's interaction with and aggregation in the presence of lipids; however, this was dependent on the lipid system.
\end{abstract}

\subsection{Introduction}

A prominent feature of many neurodegenerative diseases, e.g. Alzheimer's disease (AD), Parkinson's disease (PD), and Huntington's disease (HD), is the deposition of proteinaceous material in the form of plaques and inclusions within tissues and cellular compartments. These depositions are predominately comprised of amyloid 
that are defined by a fibrillar morphology and a cross $\beta$-sheet structure. In particular, HD is an autosomal dominant genetic disorder caused by the expansion of a polyglutamine (polyQ) region in the first exon of the huntingtin protein (htt) above a critical threshold ( 35 repeat glutamine residues), ${ }^{1}$ and this expansion promotes htt's aggregation into fibrils. ${ }^{2} \mathrm{Htt}$ aggregation is a multifaceted process involving, beyond fibrils, a variety of other aggregate species, including oligomers, annular structures, and amorphous aggregates. ${ }^{2-4}$ Due to the heterogeneous nature of htt aggregation, debate continues about the role different aggregate species play in toxicity. In this regard, htt oligomers, ${ }^{5-}$ 7 fibrils, ${ }^{8,9}$ and inclusions ${ }^{10}$ have all been directly implicated as toxic entities.

Within the cellular environment, htt associates with a variety of membranous surfaces, e.g. mitochondria, endoplasmic reticulum (ER), the nuclear envelope, tubulovesicles, endosomes, lysosomes, and synaptic vesicles. ${ }^{11-16}$ These membranes, predominately comprised of lipids, provide subcellular environments that influence htt aggregation. ${ }^{17}$ In mouse models of HD, lipids accumulate in htt aggregates and inclusions, ${ }^{18-20}$ and htt aggregates disrupt lipid membranes ${ }^{21-24}$ and organelle integrity. ${ }^{10,15,16,25} \mathrm{Htt}$ 's interaction with membranous surfaces not only influences aggregation but may also be involved directly in toxic pathways. Several model lipid membranes influence htt aggregation in vitro in a variety of ways. ${ }^{22,23,26-31} \mathrm{POPC/POPS}$ lipid vesicles accelerate fibril formation by promoting a distinct aggregation pathway; ${ }^{32}$ however total brain lipid extract suppresses fibrilization. ${ }^{21,33}$

The first $17 \mathrm{~N}$-terminal amino acids of htt (Nt17) that directly precede the polyQ region is implicated in both htt aggregation and interaction with lipid membranes..$^{21,34}$ $\mathrm{Nt} 17$ is predominately disordered in solution but has a propensity to adopt an 
amphipathic $\alpha$-helical structure upon interaction with a binding partner. Nt17 domains of several htt proteins can associate to form $\alpha$-helical rich oligomers. ${ }^{35,36}$ These oligomers enhance fibril formation by bringing together polyQ domains of different htt proteins..$^{35,36}$ Beyond this role in promoting aggregation, the ability to form an amphipathic $\alpha$-helix facilitate the binding of htt-exon 1 to lipid membranes. A variety of post-translational modifications (PTMs) occur within Nt17, with implication for htt aggregation and membrane interactions. These PTMs include phosphorylation, ${ }^{37-40}$ oxidation, ${ }^{41,42}$ acetylation, ${ }^{30,43}$ SUMOylation, ${ }^{32-35}$ and ubiquitination. ${ }^{32-34}$ PTMs impact htt function, translocation, and toxicity. ${ }^{37,44-46}$ In particular, Nt17 contains three phosphorylatable residues: threonine 3 (T3), serine 13 (S13), and serine 16 (S16). Phosphorylation of $\mathrm{S} 13$ and S16 reduces accumulation of htt and pathogenesis in animal models, modifies htt subcellular localization, reduces aggregate stability, and inhibits fibril formation. ${ }^{37,39,47-51}$ Imitating threonine 3 (T3) phosphorylation enriches polyQ-mediated toxicity in Drosophila models of HD but increases the amount of insoluble htt-exon1 species. ${ }^{37,51}$ More recent literature on htt-exon1 peptides demonstrate that inducing phosphorylation ${ }^{50-53}$ or adding a negative charge ${ }^{51,54}$ through aspartic acid single point mutations to the $\mathrm{T} 3$ residue leads to a decrease in fibril elongation. ${ }^{51,55}$ Inhibition of fibrilization via phosphorylation also appears related to modifications in the nucleation process related to the formation of $\alpha$-helical rich oligomers facilitated by $\mathrm{Nt} 17 .{ }^{47}$

Here, the aim was to determine how phosphorylation at T3, S13, and S16 influence the ability of htt-exon1 to directly interact with lipid membranes and how these interactions influence htt aggregation. To achieve this, aggregation of htt-exon1 proteins containing phosphomimetic mutations (T3D, S13D, or S16D) in the presence and 
absence of lipid membranes was investigated by various biophysical techniques. Lipid systems investigated were TBLE, POPC, and POPG. Phosphorylation altered htt's interaction with and aggregation in the presence of lipids, but this was heavily influenced by the specific lipid systems exposed to htt.

\subsection{Materials and Methods}

2.2.1 Preparation of Phosphomimetic Constructs. Extracted DNA from a glutathione S-transferase (GST)-htt-exon1(46Q) fusion protein was inserted at cleaved sites BamH1 and Sal1 vectors within a pGEX-5X-1 plasmid. This region was replaced with three separate constructs, in which an aspartic acid residue replaced the threephosphorylation post translational modification sites within the Nt17 domain (T3D, S13D and S16D). The construct plasmids were transformed into One Shot thermocompetent STBL3 E. Coli cells and made into glycerol stocks stored at $-80^{\circ} \mathrm{C}$ for future purification in the same manner as the unmodified wild-type (WT).

\subsubsection{Expression, Purification of (GST)-htt-exon1 Fusion Protein. (GST)-htt-} exon1(46Q) fusion proteins (WT, T3D, S13D and S16D) were purified as described previously. ${ }^{56}$ In short, GST-htt-exon1 was expressed with isopropyl B-D-thiogalactoside for 4 hours at $30^{\circ} \mathrm{C}$ in E. Coli. Cells were lysed with lysozyme $(0.5 \mathrm{mg} / \mathrm{mL})$ and sonication, and fusion proteins were purified using a $5 \mathrm{~mL}$ GST affinity column and liquid chromatography (LPLC, Bio Rad). Relevant protein bands were observed with gel electrophoresis technique and Coomassie blue staining to assess purity and appropriate fractions. Protein concentration was determined using Coomassie Bradford reagent. Before each experiment, pre-existing aggregates were removed through high speed centrifugation at $20,000 \mathrm{~g}$ for 30 minutes at $4^{\circ} \mathrm{C}$. Initiation of aggregation through 
cleavage of the GST tag is performed by the addition of Factor Xa protease (New England Bio Labs). All experiments were performed in buffer A (50mM Tris- $\mathrm{HCl}, \mathrm{pH} 7$, $150 \mathrm{mM} \mathrm{NaCl})$.

2.2.3 Lipid Preparation. Total brain lipid extract (TBLE), 1-Palmitoyl-2-oleoyl-glycero-3phosphatidylcholine (POPC), and 1-Palmitoyl-2-oleoyl-sn-glycero-3-phospho-(1'-racglycerol (POPG) were purchased as lyophilized powders (Avanti Polar Lipids). Lipids were dissolved in chloroform and aliquoted for further use. Residual traces of solvent were evaporated overnight in high vacuum. Resulting lipid films were hydrated in buffer A at $60^{\circ} \mathrm{C}$ with vortex mixing until all lipid was suspended in solution. Vesicle formation was promoted by 10 freeze/thaw cycles using liquid $\mathrm{N}_{2}$ followed by bath sonication at room temperature for 1 hour.

2.2.4 Thioflavin T (ThT) Aggregation Assay. Htt-exon1(46Q) constructs were diluted to a final concentration of $20 \mu \mathrm{M}$ in the presence of $125 \mu \mathrm{M}$ ThT (Sigma-Aldrich, St. Louis, MO) and the desired lipid vesicles if needed. In experiments with vesicles, a 10:1 lipid:protein ratio was used, resulting in a lipid concentration of $200 \mu \mathrm{M}$. Assays were performed in black Costar 96-well plates with clear flat bottoms (100 $\mu \mathrm{L}$ per well), and ThT fluorescence was monitored with a microplate reader (SpectraMax M2). Experiments were run at $37^{\circ} \mathrm{C}$ with readings every 5 minutes for 18 hours at $440 \mathrm{~nm}$ excitation and $484 \mathrm{~nm}$ emission. For an individual experiment, all conditions were run in triplicate, and a minimum of three independent experiments were performed.

2.2.5 Polydiacetylene (PDA) Vesicle Binding Assay. 10,12-tricosadiynoic acid (GFS Chemicals, Columbus, $\mathrm{OH}$ ) and lipid system of choice were dissolved at a 2:3 molar ratio in a solution of 1:4 ethanol/chloroform. Solution was evaporated off under a gentle 
stream of $\mathrm{N}_{2}$, and the film was dissolved with buffer $A$ at $70^{\circ} \mathrm{C}$. The solution was sonicated for 10-minute intervals at $100 \mathrm{~W}$ using a sonic dismembrator (FisherSci) until solution was sufficiently opaque and stored at $4^{\circ} \mathrm{C}$ overnight to ensure self-assembly of the mixture into vesicles. Polymerization of the PDA was initiated by irradiation at 254 $\mathrm{nm}$ for 10 minutes at room temperature with stirring, resulting in a blue, opaque solution. The colorimetric response was monitored by UV-Vis spectroscopy to quantify protein binding to vesicles with a SpectraMax M2 microplate reader (Molecular Devices). The assay was performed in a 96 -well plate format ( $100 \mu \mathrm{L}$ total volume per well), and each condition was performed in triplicate per experiment. A minimum of three unique experiments were performed. Each plate contained negative controls (neat buffer A) and a positive control (final concentration of $100 \mu \mathrm{M} \mathrm{NaOH}$ ). The $\mathrm{NaOH}$ control was used to standardize PDA results across experiments. ${ }^{57}$ The percent colorimetric response $(\% \mathrm{CR})$ was determined every $5 \mathrm{~min}$ for 18 hours at $37^{\circ} \mathrm{C}$ by measuring the blue absorbance ( $640 \mathrm{~nm}$, Ablue) and the red absorbance (500 nm, Ared) using the following equation:

$$
\text { (Eqn. 2.1) } \quad \% C R=\left[\frac{P B_{0}-P B_{1}}{P B_{0}}\right] * 100
$$

$\mathrm{PB}$ is defined as $\mathrm{A}_{\text {blue }} /\left(\mathrm{A}_{\text {blue }}+\mathrm{A}_{\mathrm{red}}\right) . \mathrm{PB}_{0}$ is determined from the negative control, and $\mathrm{PB}_{1}$ is obtained is obtained from each experimental condition. ${ }^{58,59}$

2.2.6 Atomic Force Microscopy (AFM). For ex situ AFM experiments, htt-exon1(46Q) $(20 \mu \mathrm{M})$ was incubated in the absence or presence of lipid vesicles (10:1 lipid to protein ratio) at $37^{\circ} \mathrm{C}$ and $1400 \mathrm{rpm}$ in an orbital thermomixer. At designated time points, $2 \mu \mathrm{L}$ aliquots of each sample were deposited onto freshly cleaved mica (Ted Pella Inc., 
Redding, CA) for 1 minute, washed with $200 \mu \mathrm{L}$ of $18 \mathrm{M} \Omega$ ultrapure water, and dried under a gentle stream of filtered air. Samples were imaged using a Nanoscope V MultiMode scanning probe microscope (VEECO) equipped with a closed loop vertical engage J-scanner. Silicon-oxide cantilevers with a nominal spring constant of $40 \mathrm{~N} / \mathrm{m}$ and a resonance frequency of $\sim 300 \mathrm{kHz}$ were used. Scan rate was set at $1.99 \mathrm{~Hz}$ with cantilever drive frequencies at $10 \%$ below resonance. For in situ AFM experiments a tapping mode fluid cell with an $\mathrm{O}$ ring was equipped with a cantilever a nominal spring constant of $0.1 \mathrm{~N} / \mathrm{m}$. To prepare the TBLE bilayer, lyophilized lipid film was reconstituted in $25 \mathrm{mM}$ Tris buffer at $1 \mathrm{mg} / \mathrm{mL}$. The resulting TBLE solution underwent ten freezethaw cycles followed by 10 min of bath sonication. The resulting TBLE vesicles were directly injected into the fluid cell $(30 \mu \mathrm{L})$, and the formation of a supported lipid bilayer was monitored via continuous AFM imaging. Once a continuous $40 \mu \mathrm{m} \times 40 \mu \mathrm{m}$ patch of supported lipid bilayer formed, htt constructs that had been incubated on ice with factor Xa for $2 \mathrm{~h}$ was injected into the fluid cell to a final concentration of $10 \mu \mathrm{M}$. The lipid bilayer exposed to htt was continuously imaged. Resulting images from both ex situ and in situ were then analyzed using the MATLAB image processing toolboxes (MathWorks) as described. 60,61

\subsection{Results}

\subsubsection{Phosphomimetic mutations in Nt17 alter htt aggregation. All experiments} presented here used GST-htt-exon1 fusion proteins with 46 repeat glutamines (httexon1(46Q)). Single amino acids within $\mathrm{Nt17}$ (T2, S13, or S16) were mutated to aspartic acid to mimic phosphorylation. For convenience, the control htt-exon1(46Q) protein without mutations will be referred to as $\mathrm{htt}^{\mathrm{NT}}$, and the htt-exon1(46Q) protein with 
phosphomimetic mutations will be referred to as $\mathrm{htt}^{\top 3 \mathrm{D}}$, $\mathrm{htt}^{\mathrm{S} 13 \mathrm{D}}$, and $\mathrm{htt}^{\mathrm{S16D}}$ respectively. For all GST-htt fusion proteins, cleavage of the GST tag with Factor Xa initiates aggregation. While phosphomimetic mutations in Nt17 are known to slow fibrillization,,$^{39,47}$ the ability of the specific experimental system used here to recapitulate this feature needed to be verified. Fibril formation of each htt protein was monitored with a ThT assay (20 $\mu \mathrm{M}$ concentration, Fig. 1), and all three mutations reduced aggregation over a $9 \mathrm{~h}$ period. $\mathrm{Htt}{ }^{\top 3 \mathrm{D}}$ impeded fibril formation to the greatest extent (a $75 \%$ reduction compared to $\mathrm{htt}^{\mathrm{NT}}$ in the maximum ThT signal observed), followed by $\mathrm{htt}^{\mathrm{S} 16 \mathrm{D}}(61 \%$ reduction). Of the three mutations, S13D had the mildest impact on fibril formation, reducing the maximum ThT signal by only $26 \%$. 


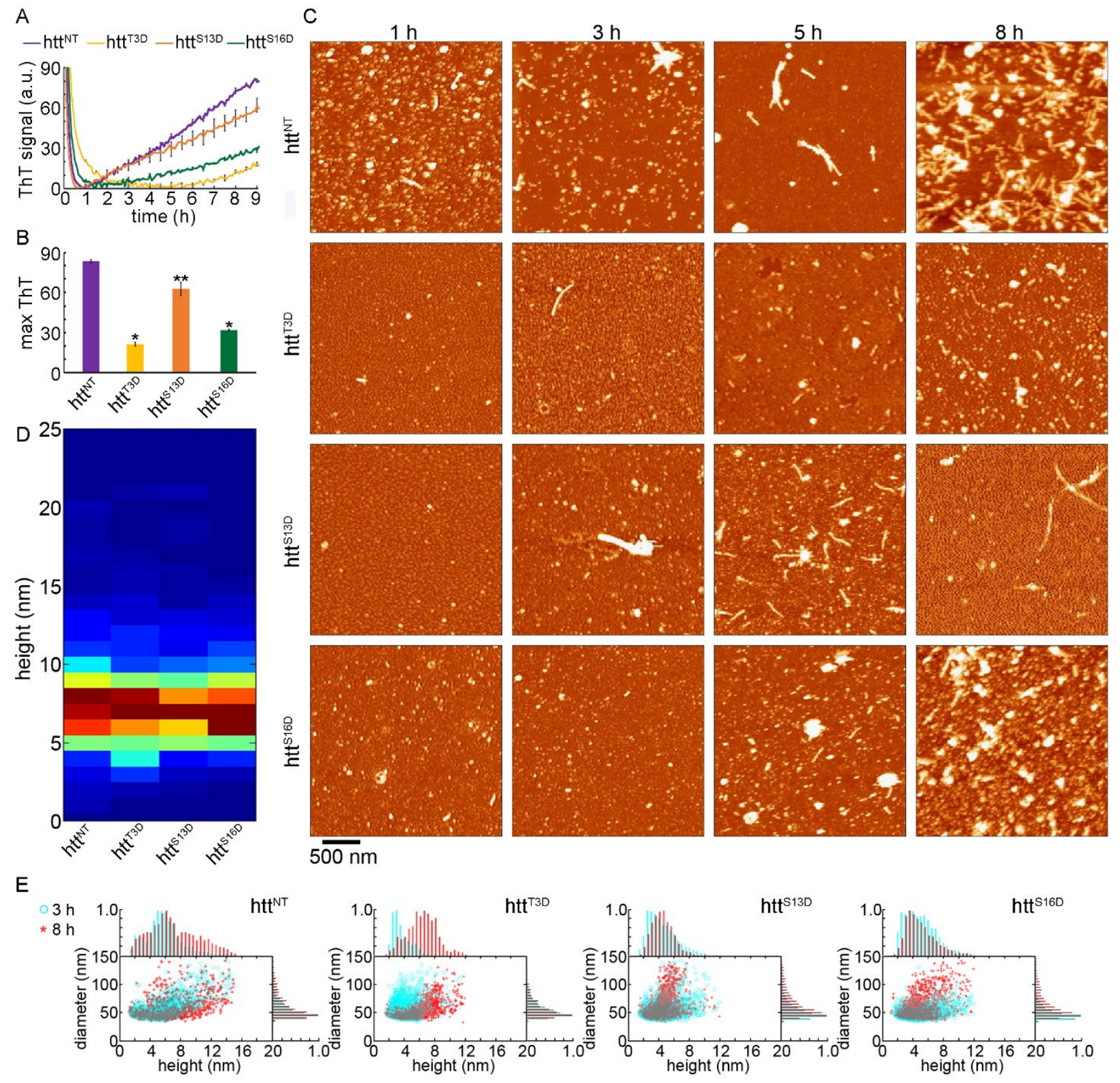

Figure 2.1: The impact of phosphomimetic mutations on htt aggregation. (A) Aggregation of $h t t^{N T}, h t t^{T 3 D}, h t t^{S 13 D}$, and $h t t^{S 16 D}$ tracked by a ThT assay. (B) Quantification of the maximum ThT fluorescent signal observed. Error bars represent SEM. * corresponds to $p<0.01$ and ** corresponds to $p<0.05$ when compared with $h t^{N T}$. (C) Representative ex situ AFM images of aggregates formed at various time points from $20 \mu \mathrm{M}$ incubations of $h t^{N T}$, htt ${ }^{T 3 D}$, htt ${ }^{S 13 D}$, and $h t t^{S 16 D}$. (D) Histograms of the average height along the contour of fibrils of $h t^{N T}, h t t^{T 3 D}, h t t^{S 13 D}$, and $h t t^{S 16 D}$ measured from AFM images. (E) Morphological correlation plots (height vs diameter) of individual oligomers observed in AFM images at 3 and $8 \mathrm{~h}$ for all four htt constructs. In addition, normalized height (top) and diameter (right) histograms are also presented. 
To further characterize the impact of phosphomimetic mutations on htt aggregation, AFM analysis was performed (Fig. 1C). $\mathrm{Htt}^{\mathrm{NT}}$, $\mathrm{htt}^{\mathrm{T} \mathrm{D}}$, $\mathrm{htt}^{\mathrm{S} 13 \mathrm{D}}$, and $\mathrm{htt}^{\mathrm{S} 16 \mathrm{D}}$ were incubated $\left(20 \mu \mathrm{M}, 37^{\circ} \mathrm{C}\right)$, sampled at $1,3,5$, and $8 \mathrm{~h}$, and imaged by AFM. For $\mathrm{htt}^{\mathrm{NT}}$, a few short fibrils were observed after $1 \mathrm{~h}$ of incubation, and these fibrils grew in number and length with time. After $8 \mathrm{~h}$, an extensive number of htt ${ }^{\mathrm{NT}}$ fibrils were observed. With each phosphomimetic mutation, fewer fibrils were observed compared with $\mathrm{htt}^{\mathrm{NT}}$, and consistent with the ThT assay, $\mathrm{htt}^{\mathrm{S} 13 \mathrm{D}}$ had the smallest impact on fibrillization. The length of fibrils observed from htt constructs containing phosphomimetic mutations tended to be shorter compared to $\mathrm{htt}^{\mathrm{NT}}$ due to the reduced rate of fibril formation. However, previous ssNMR studies demonstrates that phosphomimetic mutations in Nt17 do not significantly alter the underlying htt fibril structure. ${ }^{47}$ That is, the polyQ core was unchanged even though the rate of formation and aggregate stability is altered. To assess if fibrils formed from the four htt constructs used here may have similar underlying structure, the average height along the contour of all fibrils observed in AFM images was determined and used to construct histograms (Fig. 1D). This measurement was chosen as even though the length of fibrils may be different, if the underlying structure is similar the thickness (measured as height along the contour) would be the same, and differences in the average height along the contour of amyloid fibrils are associated with polymorphic fibril structures associated with other amyloid proteins. ${ }^{62}$ Fibrils of all four constructs had similar average heights (mode of $\sim 7 \mathrm{~nm}$ ). The average height along fibril contours observed here is consistent with the phosphomimetic mutations not altering the underlying fibril core structure. 
Beyond fibrils, htt aggregates into a heterogenous mixture of oligomers. To determine if phosphomimetic mutations in Nt17 altered oligomer morphology, the height and diameter of oligomers was measured after 3 and $8 \mathrm{~h}$ of incubation using automated scripts in MATLAB. For this analysis, oligomers were defined as any feature more than $0.8 \mathrm{~nm}$ in height with an aspect ratio (longest distance across to shortest distance across) $<3.0$, indicating a globular structure. Correlation plots of diameter vs height, as well as histograms for each dimension, were constructed (Fig. 1E). $\mathrm{Htt}^{\mathrm{NT}}$ formed a heterogeneous mixture of oligomers, which is particularly pronounced in the height histograms. While htt ${ }^{\mathrm{NT}}$ oligomers were diverse in morphology, the mode height was 5$7 \mathrm{~nm}$ at $3 \mathrm{~h}$ with a slight shift to larger oligomers at $8 \mathrm{~h}$. For example, there was a sizeable population of $\mathrm{ht}^{\mathrm{NT}}$ oligomers larger than $10 \mathrm{~nm}$ in height after $8 \mathrm{~h}$. Oligomers formed from phosphomimetic htt constructs displayed less heterogeneity. In general, oligomers formed from htt containing phosphomimetic mutations tended to be smaller, which may be consistent with previous reports that Ser-to-Asp mutations in htt reduce its ability to form $\alpha$-helix rich oligomers. ${ }^{47} \mathrm{Htt}^{\mathrm{T} 3 \mathrm{D}}$ formed oligomers with a mode height of 2.5-3.5 $\mathrm{nm}$ at $3 \mathrm{~h}$. There was an abrupt shift to larger oligomer species (mode height of 6-8nm); however, only a few $\mathrm{htt}^{\top 3 \mathrm{D}}$ oligomers grew beyond $10 \mathrm{~nm}$ in height. Both $\mathrm{htt}^{\mathrm{S13D}}$ and $\mathrm{htt}^{\mathrm{S} 16 \mathrm{D}}$ formed smaller oligomers than $\mathrm{htt}^{\mathrm{NT}}$ at $3 \mathrm{~h}$ (mode heights of 2.5-4 $\mathrm{nm}$ and 2.5-6 $\mathrm{nm}$ respectively). While $\mathrm{htt}^{\mathrm{S13D}}$ displayed a small shift in oligomer height at $8 \mathrm{~h}$ (mode height of 4-6 nm), the predominant morphological changes associated with $\mathrm{htt}^{\mathrm{S} 13 \mathrm{D}}$ and $\mathrm{htt} \mathrm{t}^{\mathrm{S16D}}$ at $8 \mathrm{~h}$ was a broadening of oligomers that manifested as a slight shift (development of a tail) in the diameter histograms. Collectively, these observations suggest that phosphomimetic mutations impact htt aggregation at the oligomeric stage. 
As $\alpha$-helical rich oligomers mediated by Nt17 are implicated in mechanisms to nucleate fibrillization, ${ }^{35,36}$ reduction in fibril formation associated with phosphorylation may be due to differences in efficiency of formation and types of oligomers formed.

\subsubsection{Phosphomimetics of htt alter aggregation in the presence of lipids. Having} established an experimental system, investigations aimed at elucidating the role of phosphorylation on htt/lipid interactions were performed. For these studies, three lipid systems were chosen as lipid composition can heavily influence amyloid formation. The first lipid system was total brain lipid extract (TBLE). TBLE has been shown to alter htt aggregation by suppressing fibril formation 21,33 and consists of a physiologically relevant mixture of lipid components. In addition, two simpler lipid systems were investigated (POPC and POPG). With the phosphomimetic constructs neutralizing the net positive charge of Nt17, these two simplified systems were chosen because they have the same tails but differently charged head groups, as POPC is zwitterionic and POPG is anionic.

To assess the impact of the three lipid systems on fibrillization of the four htt constructs, ThT assays were performed in the presence of lipid vesicles (Fig. 2). For these assays, the lipid to $\mathrm{htt}$ molar ratio was $20: 1$, and all vesicles were in the range of $140-180 \mathrm{~nm}$ in diameter as assessed by dynamic light scattering. Consistent with previous reports, ${ }^{21,33}$ TBLE reduced $\mathrm{htt}^{\mathrm{NT}}$ fibrillization relative to the absence of lipid (compare the maximum ThT signals in Figure 2A to Figure 1B). This reduction in fibrillization associated with the presence of TBLE vesicles was also apparent for the three phosphomimetic htt constructs. Relative to $\mathrm{htt}^{\mathrm{NT}}$ in the presence of TBLE, the phosphomimetic mutations further reduced fibrillization with the same pattern as observed in the absence of lipids. That is, $\mathrm{htt}^{\mathrm{T}}{ }^{3 \mathrm{D}}$ produced the smallest ThT signal $(77 \%$ 
reduction compared to $\mathrm{htt}^{\mathrm{NT}}$ in the presence of TBLE), followed by $\mathrm{htt}^{\mathrm{S} 16 \mathrm{D}}(54 \%$ reduction), and $\mathrm{htt}^{\mathrm{S} 13 \mathrm{D}}$ (29\% reduction).
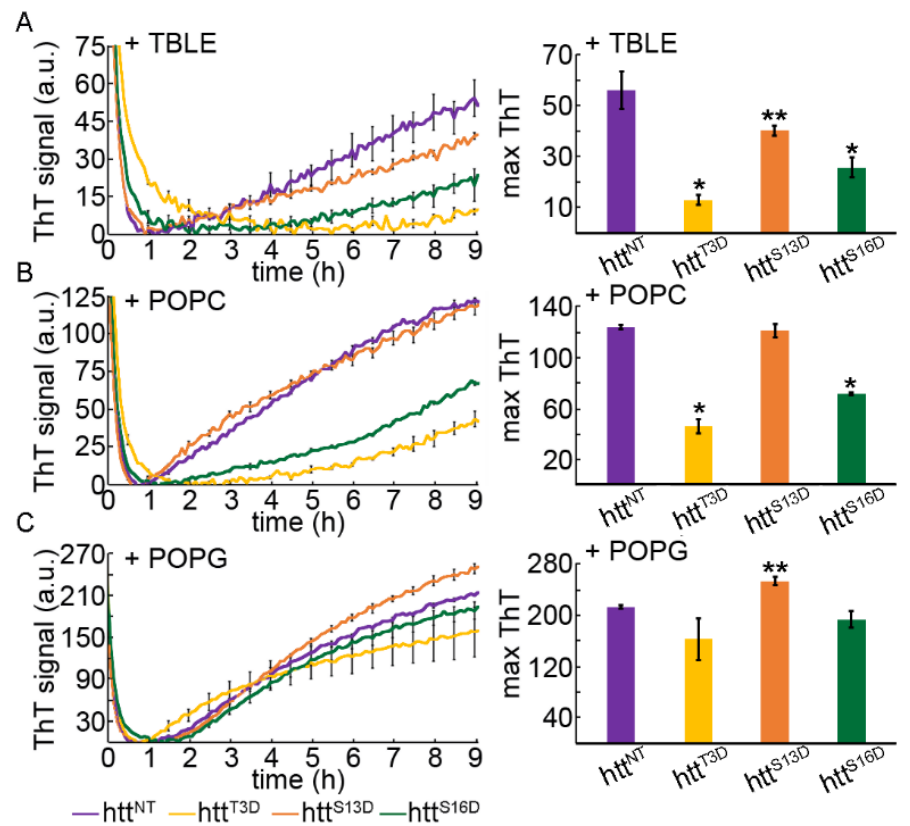

Figure 2.2. Phosphomimetic mutations influence htt aggregation in the presence of lipid vesicles. ThT assays and quantification of the maximum ThT fluorescent signal observed for $h t t^{N T}, h t t^{T 3 D}$, $h t t^{S 13 D}$, and $h t t^{S 16 D}$. Error bars represent SEM in the presence of vesicles comprised of (A) TBLE, (B) POPC, and (C) POPG. * corresponds to $p<0.01$ and ${ }^{* *}$ corresponds to $p<0.05$ when compared with $\mathrm{htt}^{N T}$.

With the addition of POPC or POPG vesicles, htt ${ }^{\mathrm{NT}}$ fibrillization was enhanced compared with aggregation reactions in the absence of lipids (compare Figure 2B-C with Figure 1B), with POPG having a significantly larger effect. The fibrillization of the phosphomimetic htt constructs was also enhanced in the presence of POPC or POPG, and the extent of fibrillization continued to follow the same pattern. That is, $\mathrm{htt}^{\mathrm{T}} \mathrm{D}$ aggregated the least, followed by $\mathrm{htt}^{\mathrm{S} 16 \mathrm{D}}$ and $\mathrm{htt}{ }^{\mathrm{S} 13 \mathrm{D}}$ respectively. However, the relative inhibition of fibrillization with respect to $\mathrm{htt}^{\mathrm{NT}}$ aggregation in the presence of POPC or POPG was reduced. With POPC, there was no significant difference between the ThT 
signal produced by $\mathrm{htt}^{\mathrm{NT}}$ and $\mathrm{htt}^{\mathrm{S} 13 \mathrm{D}}$. With $\mathrm{POPG}$, the ThT signal was not significantly different between $\mathrm{htt}^{\mathrm{NT}}$ and $\mathrm{htt}^{\mathrm{S} 16 \mathrm{D}}$. Furthermore, there was a significant increase in ThT signal in $\mathrm{htt}^{\mathrm{S} 13 \mathrm{D}}$ compared to $\mathrm{htt}^{\mathrm{NT}}$ in the presence of POPG.

To further establish the impact of the three lipid systems on the aggregation of the htt constructs, AFM analysis was performed. As before, incubation experiments were performed with fresh preparations $(20 \mu \mathrm{M})$ of $\mathrm{htt}^{\mathrm{NT}}$, $\mathrm{htt}^{\mathrm{T} \mathrm{D}}$, htt ${ }^{\mathrm{S} 13 \mathrm{D}}$, and $\mathrm{htt}{ }^{\mathrm{S} 16 \mathrm{D}}$, but this time they were incubated with vesicles of TBLE, POPC, or POPG (20:1 lipid to peptide ratio). Background images of these lipid systems deposited on mica without htt are shown in Figure 3. These incubations were sampled at 1, 3, 5, and $8 \mathrm{~h}$.
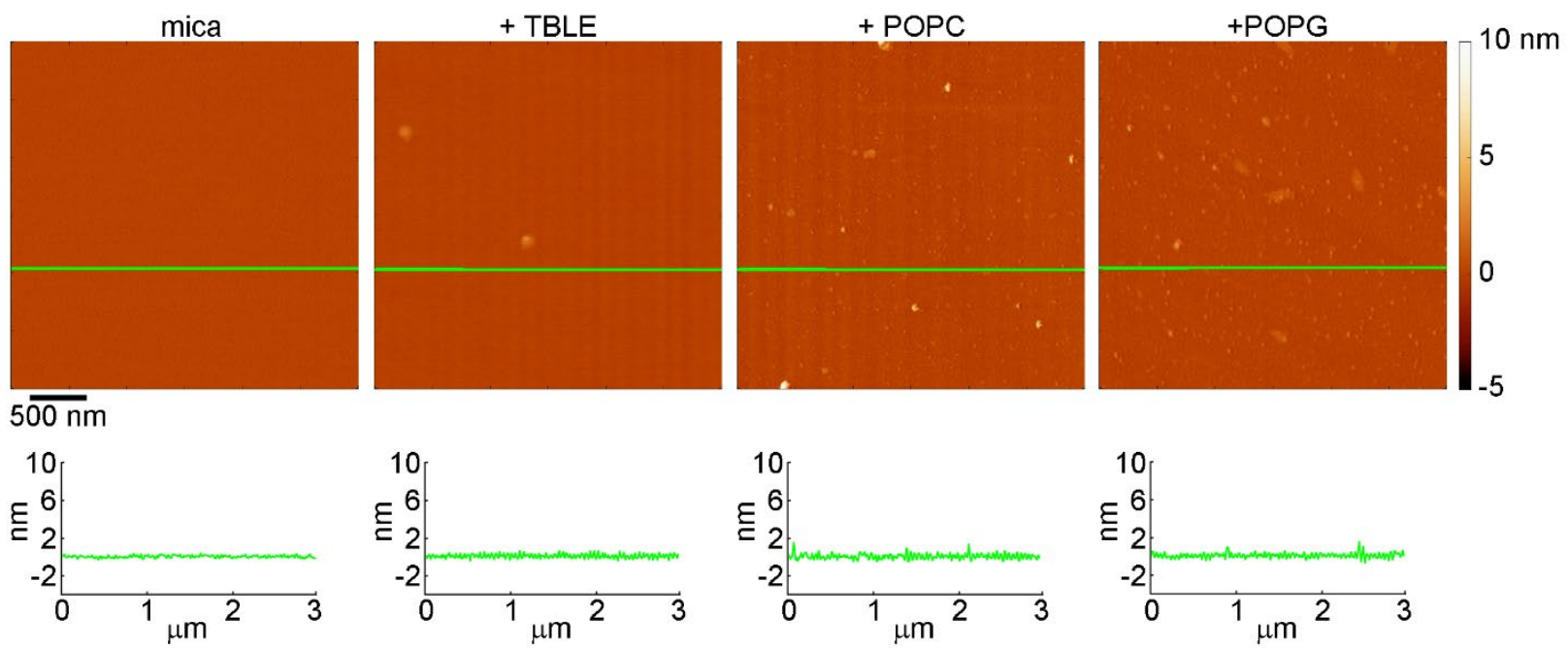

Figure 2.3. Representative AFM images of backgrounds associated with deposited solutions of lipid vesicles. Clean mica and mica exposed to TBLE, POPC, or POPG vesicles are compared. The green line in each image corresponds to the height profile shown directly below each image.

Representative AFM images of aliquots removed from these solutions after $8 \mathrm{~h}$ of incubation are shown in Figure 4. Representative AFM images of the other three time points for incubations with TBLE, POPC, or POPG vesicles are shown as Figures 5, 6, 
and 7 respectively. A variety of aggregates (oligomers and fibrils) are observed on mica under all conditions. The average height along the contour of fibrils was measured to determine if the presence of the lipids altered fibril structure (Fig. 4B). Despite different rates of formation, there were not any significant differences in fibril structures across the four htt constructs within a single lipid system. However, the fibril structure appeared altered by POPG. With TBLE and POPC, the average height along the contour of the fibril was $\sim 6-7 \mathrm{~nm}$, which is consistent with fibrils formed in the absence of lipids. With POPG, the average height along the contour ranged from $3-5 \mathrm{~nm}$, suggesting that the structure of fibrils formed in the presence of POPG may vary from fibrils formed in the absence of lipid.

A
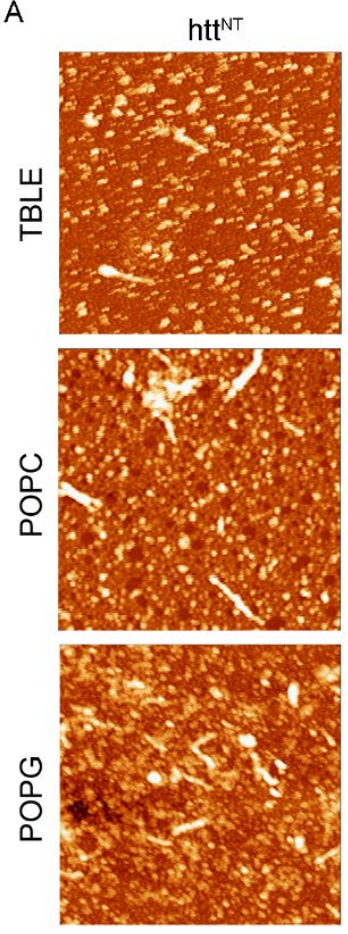

$5 \overline{00} \mathrm{~nm}$
$\mathrm{htt}^{\mathrm{T3D}}$
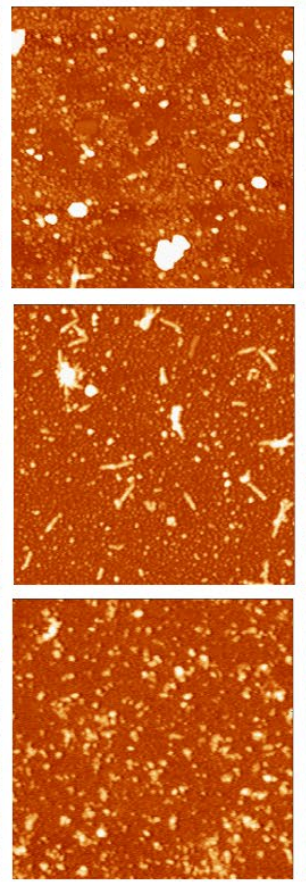
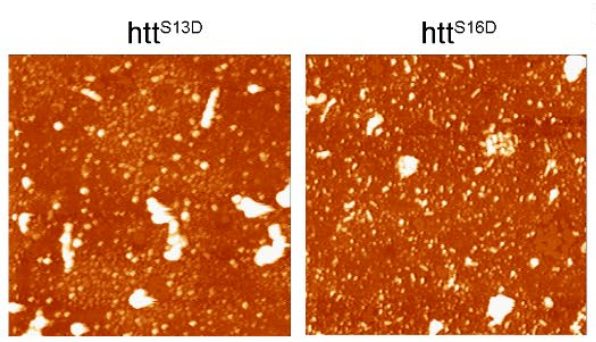

$\mathrm{B}$
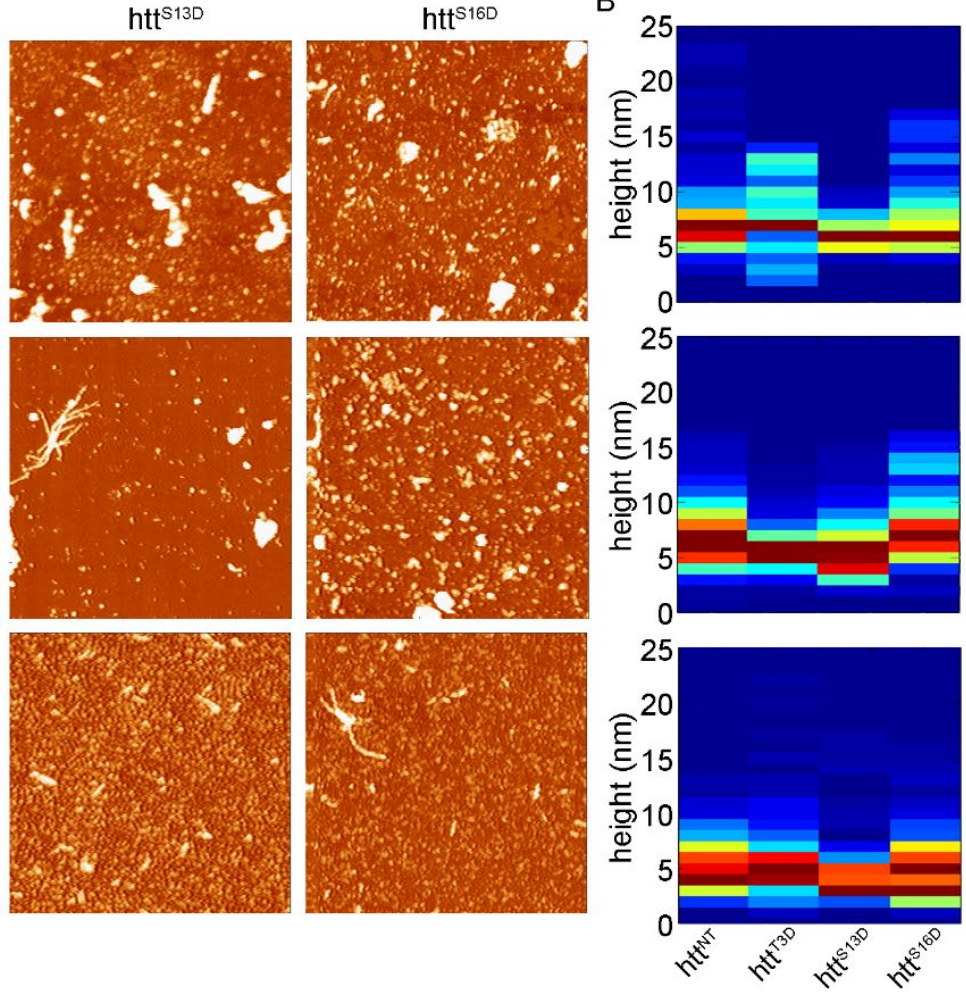

Figure 2.4. Phosphomimetic htt constructs form a variety of aggregates in the presence of lipid vesicles. (A) Representative ex situ AFM images of aggregates formed after $8 \mathrm{~h}$ of incubation (for other time points see figures S2-S4) from $20 \mu \mathrm{M}$ incubations of $\mathrm{htt}^{\mathrm{NT}}$, htt ${ }^{T 3 D}$, 
$h t t^{S 13 D}$, and $h t^{S 16 D}$ in the presence of vesicles comprised of TBLE, POPC, or POPG. (B) Histograms of the average height along the contour of fibrils of $h t^{N T}, h t t^{T 3 D}$, htt ${ }^{S 13 D}$, and $h t^{S 16 D}$ formed in the presence of vesicles comprised of TBLE, POPC, or POPG measured from AFM images.

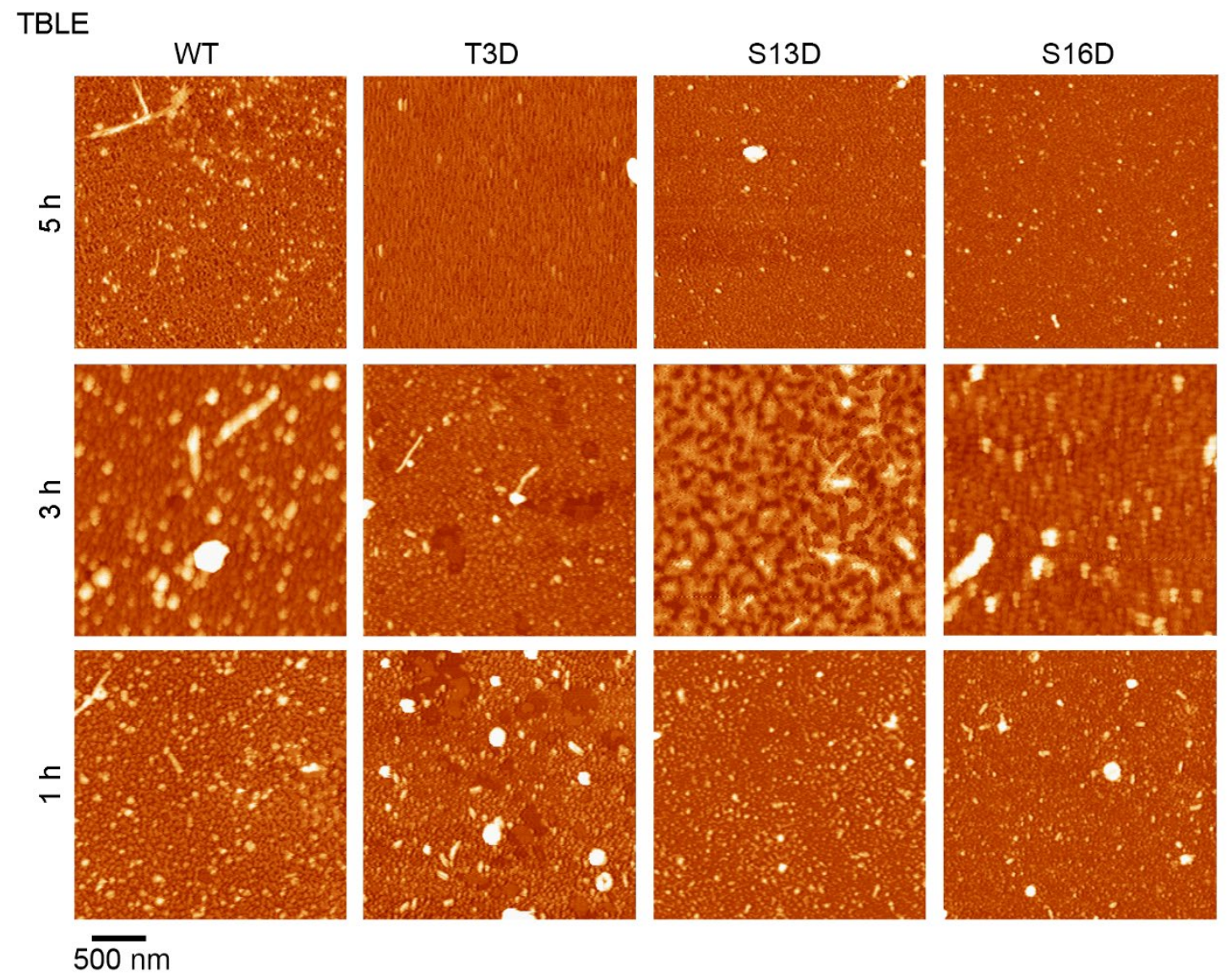

Figure 2.5. Phosphomimetic construct aggregates observed with ex situ AFM in the presence of TBLE. Representative ex situ AFM images of aggregates formed after 1, 3, and 5 $h$ of incubation from $20 \mu \mathrm{M}$ incubations of httNT, httT3D, httS13D, and httS16D in the presence of TBLE. 


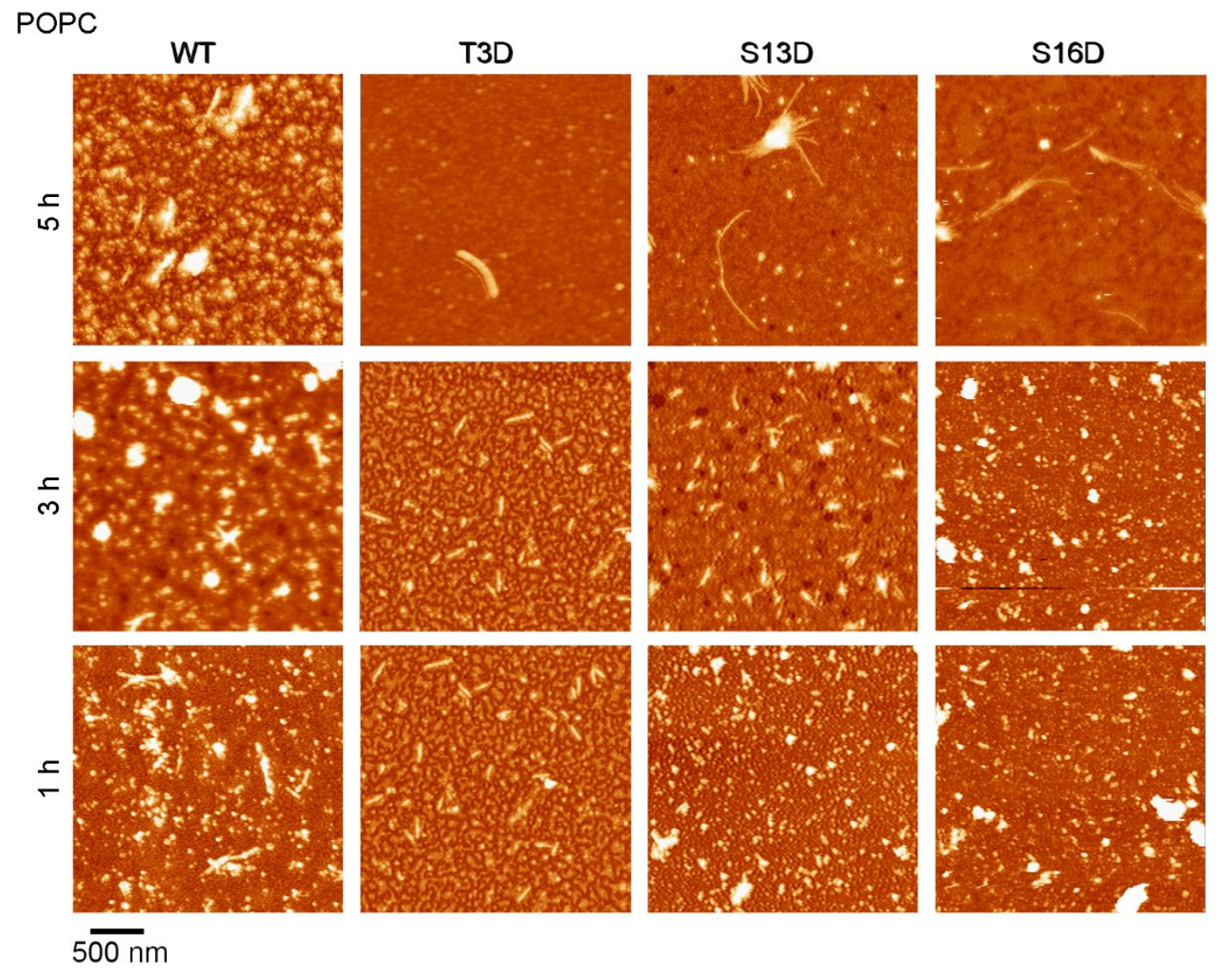

Figure 2.6. Phosphomimetic construct aggregates observed with ex situ AFM in the presence of POPC. Representative ex situ AFM images of aggregates formed after 1, 3, and 5 $h$ of incubation from $20 \mu \mathrm{M}$ incubations of $h \mathrm{tt}^{\mathrm{NT}}$, $h \mathrm{tt}^{\mathrm{T} 3 \mathrm{D}}$, $h \mathrm{tt}^{\mathrm{S} 13 \mathrm{D}}$, and $\mathrm{htt} \mathrm{t}^{\mathrm{S16D}}$ in the presence of POPC. 


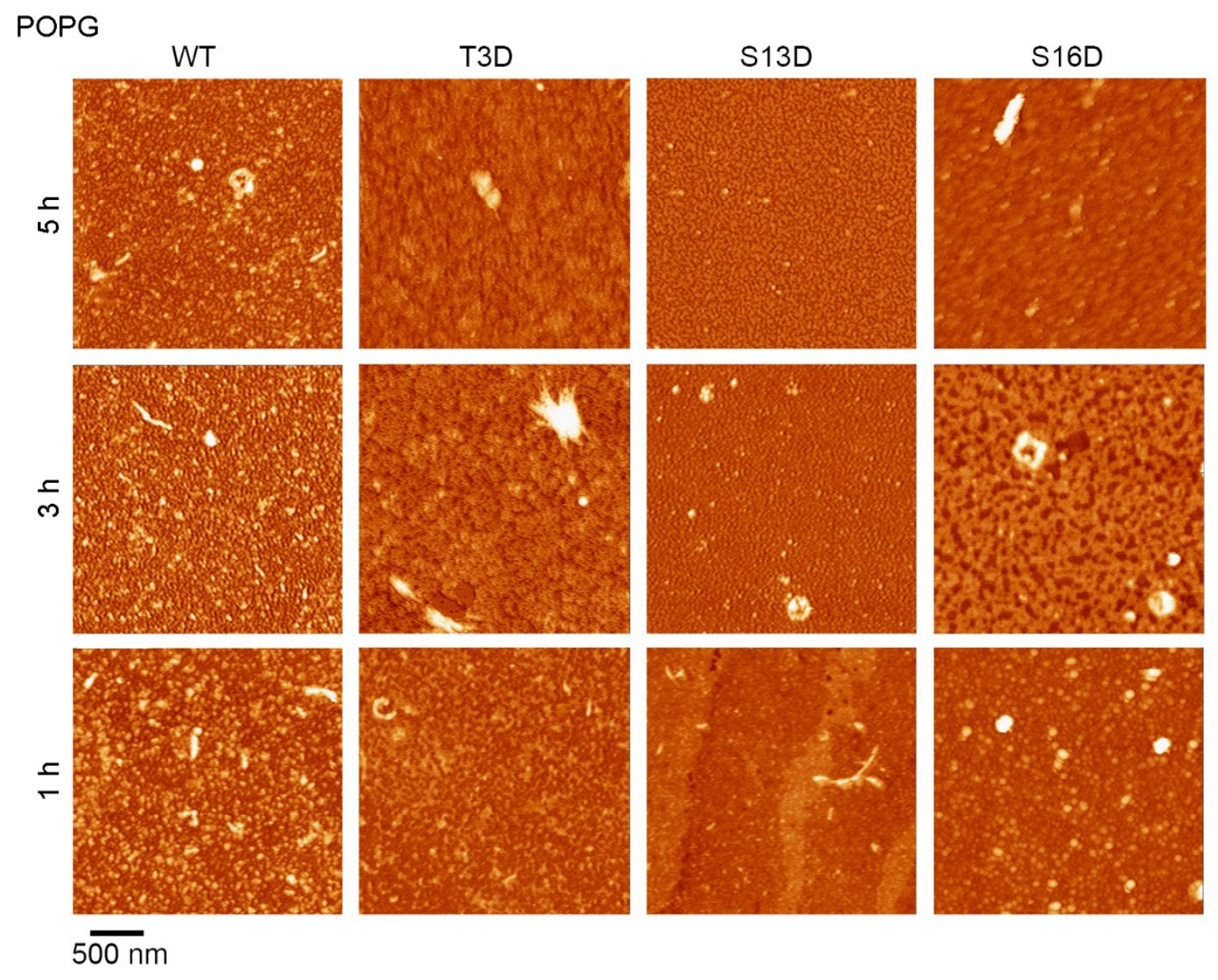

Figure 2.7. Phosphomimetic construct aggregates observed with ex situ AFM in the presence of POPG. Representative ex situ AFM images of aggregates formed after 1, 3, and 5 $h$ of incubation from $20 \mu \mathrm{M}$ incubations of $\mathrm{htt}^{\mathrm{NT}}$, htt $\mathrm{HID}^{\mathrm{T} D}, \mathrm{htt}^{\mathrm{S13D}}$, and $\mathrm{htt}^{\mathrm{S} 16 \mathrm{D}}$ in the presence of POPG.

Using the same criteria as before, the height and diameters of oligomers observed at 3 and $8 \mathrm{~h}$ of incubation were determined and used to build correlation plots and histograms (Fig. 8). The presence of all three lipid systems reduced the heterogeneity of htt ${ }^{\mathrm{NT}}$ oligomers at a given time point. With TBLE, htt ${ }^{\mathrm{NT}}$ oligomers were less heterogeneous than in the absence of lipids, displaying mode heights of 5-7.5 nm at 3 and $8 \mathrm{~h}$. Phosphomimetic mutations promoted smaller oligomers than htt ${ }^{\mathrm{NT}}$ in the presence of TBLE, with mode heights of 2-3.5 nm, 2-3 nm, and 2-6 nm for $\mathrm{htt}^{\top 3 \mathrm{D}}$, htt ${ }^{\mathrm{S13D}}$, and $\mathrm{htt}^{\mathrm{S} 16 \mathrm{D}}$ respectively. After $8 \mathrm{~h}$, the height distribution of oligomers formed from all phosphomimetic mutations shifted to larger size with increased heterogeneity. After $3 \mathrm{~h}$ 
of incubation, POPC vesicles promoted small oligomers with all four htt constructs (mode heights of 2.5-4 nm, 2-3.5 nm, 2-3 nm, and 2-3 nm for htt ${ }^{\mathrm{NT}}$, htt ${ }^{\top 3 \mathrm{D}}$, htt ${ }^{\mathrm{S} 13 \mathrm{D}}$, and $\mathrm{htt}^{\mathrm{S} 16 \mathrm{D}}$ respectively). While oligomers formed from $\mathrm{htt}^{\mathrm{NT}}$ and $\mathrm{htt}^{\top 3 \mathrm{D}}$ in the presence of POPC remained relatively small, oligomer formed from $\mathrm{htt}^{\mathrm{S} 13 \mathrm{D}}$ and $\mathrm{htt} \mathrm{t}^{\mathrm{S} 16 \mathrm{D}}$ were significantly larger at $8 \mathrm{~h}$ (mode heights of $\sim 6-8 \mathrm{~nm}$ for both). POPG initially promoted smaller oligomers with all four htt constructs (mode heights in the $2-4 \mathrm{~nm}$ range at $3 \mathrm{~h}$ ); however, a population of larger oligomers appeared with POPG after $8 \mathrm{~h}$ for all four constructs, resulting in a more heterogeneous population. This shift was the most pronounced with $\mathrm{htt}^{\mathrm{S} 16 \mathrm{D}}$.

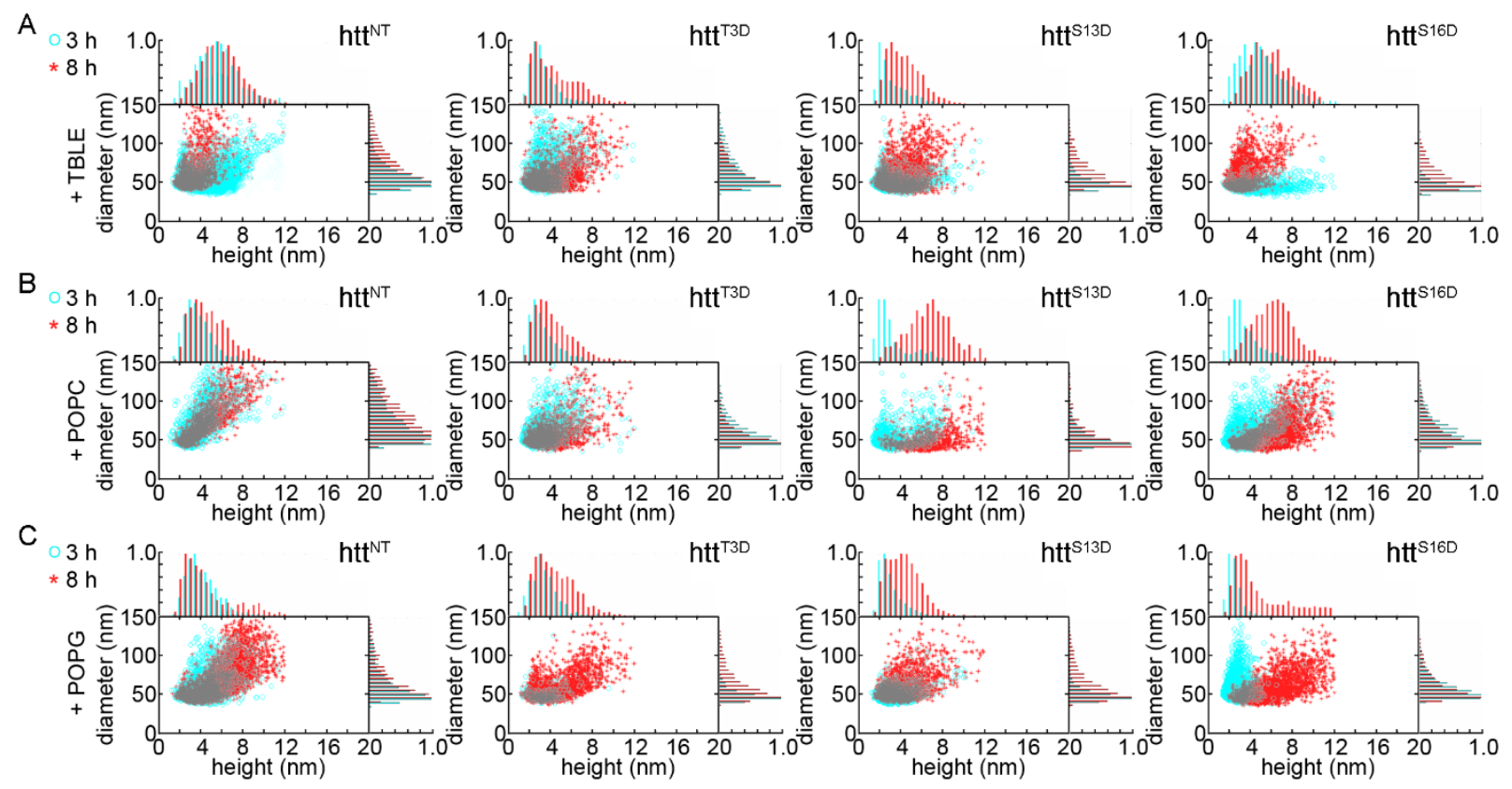

Figure 2.8. Lipids promote unique htt and phosphomimetic htt oligomer morphologies. Morphological correlation plots (height vs diameter) of individual oligomers observed in AFM images at 3 and $8 \mathrm{~h}$ for all four htt constructs in the presence of vesicles comprised of (A) TBLE, (B) POPC, and (C) POPG. In addition, normalized height (top) and diameter (right) histograms are also presented. 


\subsubsection{Phosphomimetics of htt impact binding in a lipid specific manner. As the}

presence of lipid vesicles altered htt aggregation (with and without phosphomimetic mutations) in a lipid specific manner, a series of colorimetric membrane binding assays using lipid/polydiacetylene (PDA) vesicles were performed to determine the impact of phosphomimetic mutations on membrane binding. These vesicles were made with either TBLE, POPC, or POPG as the lipid component. Lipid/PDA vesicles have varied colorimetric responses (CRs) when exposed to proteins or small molecules depending on the extent of interaction. ${ }^{58,59}$ The $\mathrm{CR}$ in the lipid/PDA vesicles is related to transitions of the PDA polymer backbone structure, which is affected by changes in tension associated with exogenous molecules interacting with and/or inserting into the vesicle. ${ }^{59}$ By measurement of the absorbance of both the blue $(640 \mathrm{~nm})$ and red $(500 \mathrm{~nm})$ wavelengths of lipid/PDA vesicles upon exposure to htt-exon1(46Q), the percent CR was obtained, which directly corresponds to the protein-lipid interaction. In addition, these assays were normalized to an $\mathrm{NaOH}$ control, allowing for comparison across lipid systems. ${ }^{57}$

Phosphomimetic mutations significantly enhanced the interaction of htt with TBLE as shown in Figure 9, with $\mathrm{htt}^{\top 3 \mathrm{D}}$ and $\mathrm{htt}^{\mathrm{S} 13 \mathrm{D}}$ having the strongest interaction with the membrane $\left(\sim 2 x\right.$ the maximum \% CR compared to htt $\left.{ }^{\mathrm{NT}}, \mathrm{p}<0.01\right)$. With TBLE, the enhanced interaction of $\mathrm{htt}^{\mathrm{S} 16 \mathrm{D}}$ compared to $\mathrm{htt}^{\mathrm{NT}}$ was less pronounced and not statistically significant. In contrast, phosphomimetic mutations delayed the interaction between $\mathrm{htt}$ and POPC for the first $3.5 \mathrm{~h}$. The $\alpha \beta \alpha$ displayed by PDA/POPC vesicles exposed to $\mathrm{htt}^{\mathrm{NT}}$ reached $9-10 \%$ within 1 to $2 \mathrm{~h}$ at which time the $\% \mathrm{CR}$ leveled off to a relative steady state (max \%CR of $13.1 \%$ ). For all three phosphomimetic htt constructs 
it took $\sim 7-9 \mathrm{~h}$ to reach a similar \%CR with POPC. After the $9 \mathrm{~h}$ experiments, only $\mathrm{htt}{ }^{\mathrm{S} 16 \mathrm{D}}$ displayed a significantly smaller maximum $\% \mathrm{CR}(10.7 \%, \mathrm{p}<0.05)$ compared with $\mathrm{htt}^{\mathrm{NT}}$. Within the time frame of these experiments $\mathrm{htt}^{\mathrm{NT}}$ and $\mathrm{htt} \mathrm{t}^{\mathrm{S} 16 \mathrm{D}}$ invoked a larger \%CR with POPC vesicles compared with TBLE. Both $\mathrm{htt}^{\mathrm{T} 3 \mathrm{D}}$ and $\mathrm{htt}{ }^{\mathrm{S} 13 \mathrm{D}}$ more strongly interacted with TBLE compared to POPC. As all three phosphomimetic htt constructs had similar interactions with POPC, these cross-system differences may be due to the complexity of lipid composition of TBLE. The interaction of htt with POPG was the weakest of the three lipid systems, and there did not appear to be any significant differences associated with $\mathrm{htt}^{\mathrm{NT}}$ and the phosphomimetic htt constructs.

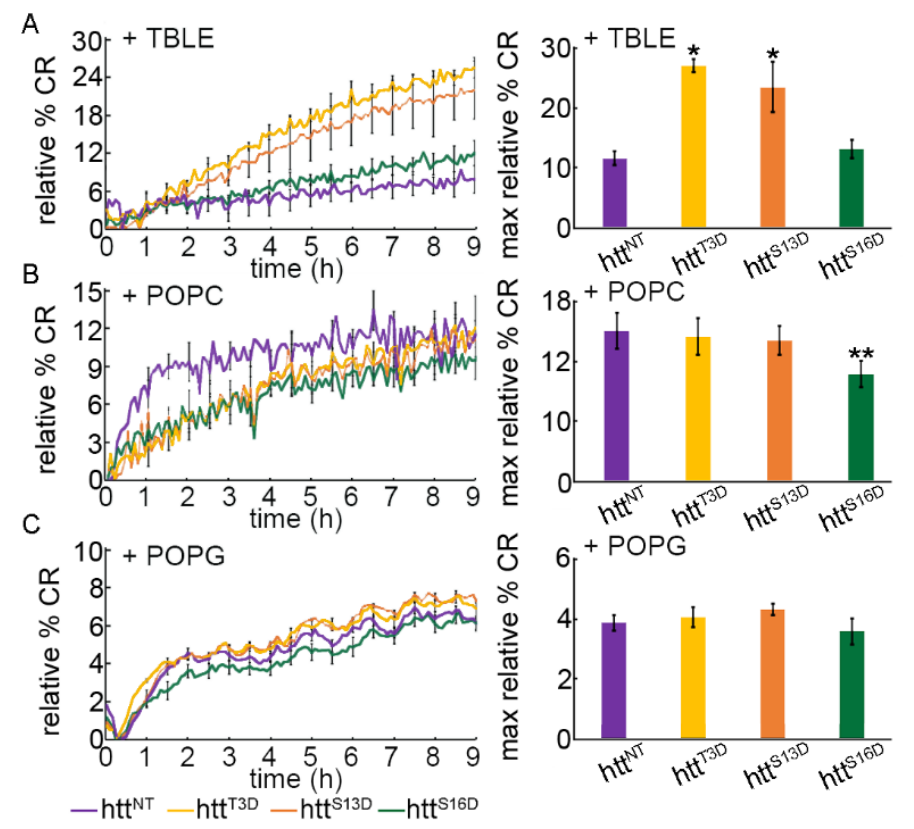

Figure 2.9. Phosphomimetic mutations in htt alter its ability to bind a variety of lipid vesicles. Normalized PDA/lipid binding assays and the maximum relative \%CR associated with exposure of PDA vesicles with the lipid component comprised of (A) TBLE, (B) POPC, or (C) $P O P G$ exposed to the four htt constructs. Error bars represent SEM. * corresponds to $p<0.01$ and ${ }^{* *}$ corresponds to $p<0.05$ when compared with $h t^{N T}$. 
2.3.4 Phosphomimetics of htt influence disruption of lipid bilayers. While the three lipid systems impacted htt aggregation as demonstrated by ThT and ex situ AFM assays, both techniques monitor bulk aggregation within an incubation. That is, they do not distinguish between htt aggregation occurring on a membrane surface or in bulk solution. In addition, there did not appear to be a direct correlation between the extent of htt/lipid interaction as measured by PDA and fibrillization associated with the phosphomimetic htt constructs. As htt aggregation occurs via a unique mechanism on POPC/POPS lipid surfaces, ${ }^{32}$ it was hypothesized that phosphomimetic mutations may be impacting aggregation at membrane surfaces in a distinct manner that is masked by the bulk aggregation assay already employed. As a result, in situ AFM was employed to directly observe aggregation of the four htt constructs directly on lipid membranes.

For these assays it is important to obtain stable, supported lipid membranes that are free of nanoscale defects. Unfortunately, such supported membranes were not reproducibly produced for the pure POPC or POPG systems. Supported TBLE bilayers were easily produced and were systematically exposed to the four htt constructs (final htt concentration of $10 \mu \mathrm{M}$ in the fluid cell). Upon injection of htt, TBLE bilayers were continuously imaged to directly observe changes in morphology and the development of htt aggregates (Figure 10). Such supported bilayers of TBLE have been extensively used to investigate their interaction with amyloid-forming proteins via in situ AFM. ${ }^{62-66}$ Across trials, the total concentration of lipid in the AFM fluid cell was maintained; however, observations were restrained to a $30 \times 30 \mu \mathrm{m}$ patch of supported bilayer that was verified to be defect-free (at the level of resolution of the AFM) prior to exposure to any htt constructs. Prior to exposure to htt, TBLE bilayers had a smooth morphology 
(RMS roughness $\sim 0.2-0.4 \mathrm{~nm}$ ) and can be continuously imaged by AFM for at least 24

$\mathrm{h}$ without inducing any morphological changes.

A

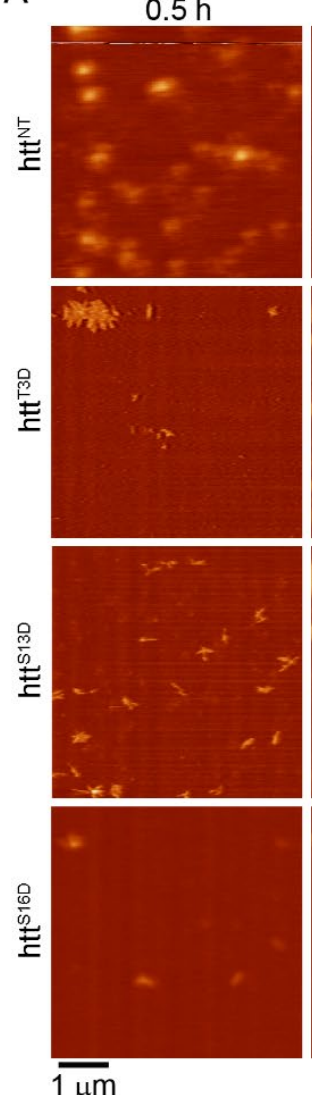

B

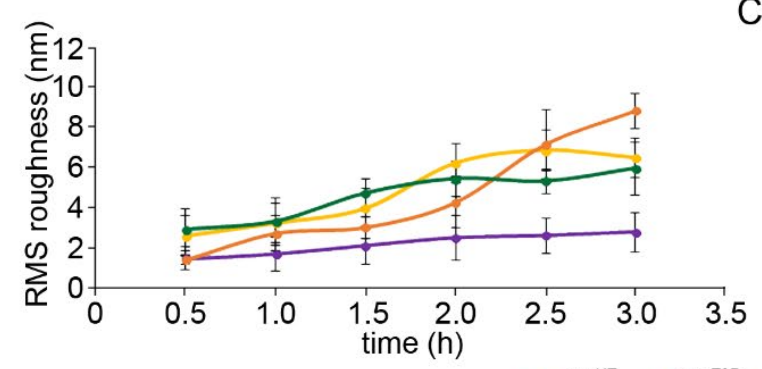

$1.5 \mathrm{~h}$
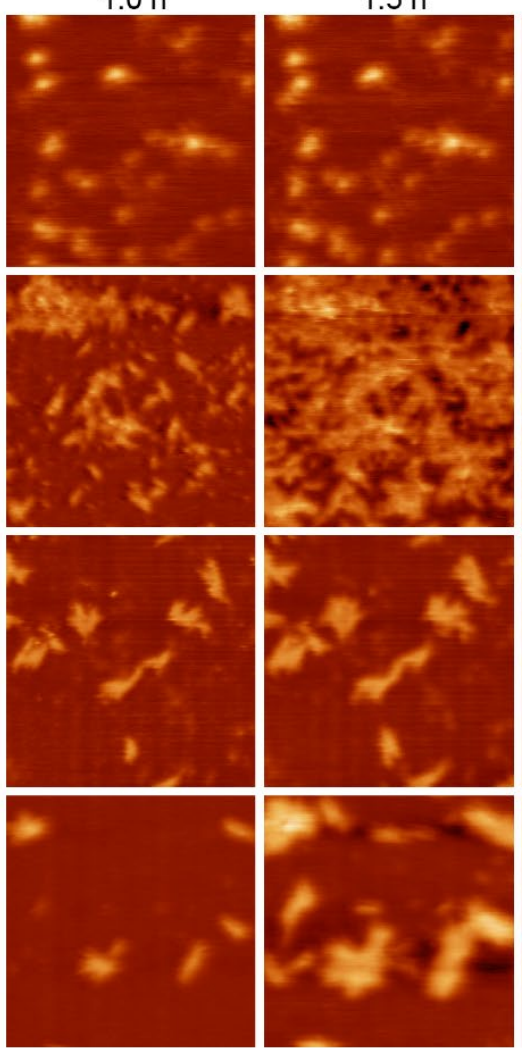

C
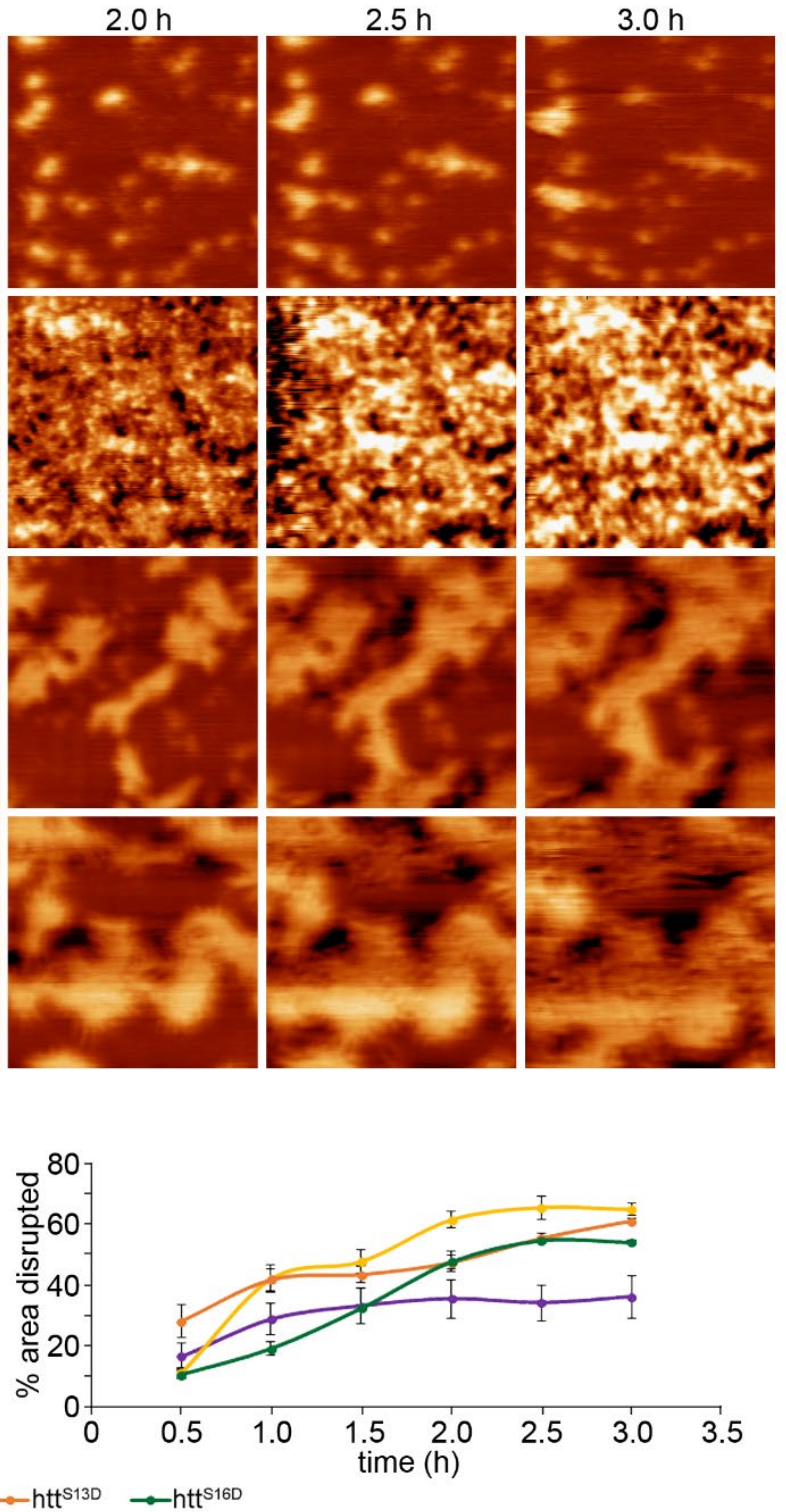

Figure 2.10. Phosphomimetic mutations alter htt aggregation on the surface of a TBLE bilayer. (A) In situ AFM images of the same region of supported TBLE bilayers exposed to 10 $\mu M h t t^{N T}, h t t^{T 3 D}, h t t^{13 D}$, or $h t t^{S 16 D}$ as a function of time. Black arrows indicate the same region in consecutive images. Quantification of (B) the RMS roughness of regions of altered morphology and (C) the \% area disrupted of supported TBLE bilayers exposed to phosphomimetic htt constructs as a function of time. Error bars represent SEM. 
Supported TBLE bilayers were systematically exposed to $\mathrm{htt}^{\mathrm{NT}}$, htt ${ }^{\mathrm{T} 3 \mathrm{D}}, \mathrm{htt}^{\mathrm{S} 13 \mathrm{D}}$, or $\mathrm{htt}^{\mathrm{S} 16 \mathrm{D}}$ at a final htt concentration within the fluid cell of $10 \mu \mathrm{M}$. Protein aggregation on the bilayer was directly monitored by continuous in situ AFM imaging for $3 \mathrm{~h} \mathrm{(Fig.} \mathrm{10).}$ To quantify the extent of morphological changes on TBLE bilayers associated with exposure to htt constructs, the RMS roughness of the altered areas on the bilayer was determined and the \% area of the bilayer displaying altered morphology (Fig. 10B-C).

Upon initial exposure of the TBLE bilayer to $\mathrm{htt}^{\mathrm{NT}}$, discrete patches displaying a roughened morphology developed on the TBLE bilayer. These patches often contained features reminiscent of htt oligomers; however, fibrillar morphology was rarely observed. With time, the area of the bilayer altered by exposure to $\mathrm{htt}^{\mathrm{NT}}$ increased $(16 \pm 4 \%$ after $0.5 \mathrm{~h}$ of exposure to $36 \pm 7 \%$ after $3 \mathrm{~h}$ ). The roughness associated with these altered regions doubled within the same time frame $(1.4 \pm 0.6 \mathrm{~nm}$ at $0.5 \mathrm{~h}$ to $2.8 \pm 1.0 \mathrm{~nm}$ after 3 h). In comparison, htt ${ }^{\top 3 \mathrm{D}}$ deposited more aggressively on the TBLE bilayer, significantly altering its morphology. Initially, $\mathrm{htt}^{\top 3 \mathrm{D}}$ appeared to form patches that resembled densely packed small fibrils. However, with time these patches became granular in appearance (RMS roughness of $6.5 \pm 1.3 \mathrm{~nm}$ by $3 \mathrm{~h}$ ) and dominated the image, covering $65 \pm 2 \%$ of the surface. $\mathrm{Htt}^{\mathrm{S} 13 \mathrm{D}}$ initially appeared as discrete fibrillar aggregates on TBLE bilayers. $\mathrm{Htt}^{\mathrm{S} 13 \mathrm{D}}$ did not develop the granular morphology observed with $\mathrm{htt}{ }^{\mathrm{T} \mathrm{D}}$; rather, large regions of altered morphology protruding above the unperturbed bilayer regions developed. These regions were relatively smooth in appearance, but because they protruded so far above the unperturbed bilayer, had the largest observed RMS roughness $(8.8 \pm 0.8 \mathrm{~nm})$ after $3 \mathrm{~h}$ of exposure to $\mathrm{htt} \mathrm{t}^{\mathrm{S13D}}$ and covered $61 \pm 1 \%$ of the surface. Exposure to $\mathrm{htt}^{\mathrm{S} 16 \mathrm{D}}$ also resulted in regions of altered morphology on the 
bilayer surface, but these regions often had fibril-like features extending from their periphery. The RMS roughness ( $5.9 \pm 1.3$ after $3 \mathrm{~h}$ ) associated with these regions was larger than $\mathrm{htt}^{\mathrm{NT}}$, but not as big as the roughness observed for the other phosphomimetic constructs. After $3 \mathrm{~h}$ of exposure, $54 \pm 1 \%$ of the bilayer surface was altered by httS16D.

The relative trend of phosphomimetic mutations increasing the area of TBLE bilayers displaying altered morphology is roughly similar to the PDA assay; however, the extent of the increased interaction appears much larger with the PDA assay. This is likely due to the more pronounced morphological changes (as quantified by RMS roughness measurements) producing larger mechanical strain on PDA vesicles, amplifying the signal. A peculiar observation is that phosphomimetic mutations appeared to promote fibrillar htt aggregates on the TBLE surface; however, the ThT assays suggested that these mutations reduced fibrillization in the presence of TBLE vesicles. This may be due to differences in what is being monitored between the two assays. ThT measures aggregation in the entirety of the incubation (solution and membrane surface); however, in situ AFM only detects changes that appear on the membrane surface. As such, this suggests that there may be distinct aggregation pathways occurring simultaneously, one in bulk solution and one at the membrane interface. Such a notion is supported by EPR studies. ${ }^{32}$

\subsection{Discussion}

PolyQ expansion drives htt aggregation and toxicity, but flanking sequences adjacent to the polyQ domain heavily influence these processes. ${ }^{67-69}$ In particular, $\mathrm{Nt}^{17}$ impacts htt localization, aggregation, and degradation in cells, $17,45,48,70$ and 
phosphorylation within Nt17 further regulates these phenomena. ${ }^{37-39,71}$ Based on its ability to form an amphipathic a-helix, a key function of Nt17 is interacting and binding lipid membranes. ${ }^{32,70}$ Here, we investigated the impact of phosphomimetic mutations within the Nt17 on htt interaction and aggregation in the presence of a variety of model lipid membranes. In general, phosphomimetic mutations at T3, S13, or S16 slowed fibril formation in the presence of lipids; however, this was highly dependent on the specific lipid system used. As aberrant htt/lipid interactions and subcellular membrane damage are observed in HD, understanding how PTMs like phosphorylation impact htt/lipid interactions could reveal new therapeutic targets and strategies.

While the current study demonstrates that altering the charge of Nt17 via phosphomimetic mutations heavily influences htt aggregation in the presence and absence of lipids, there are several caveats to these studies that need to be addressed. Phosphomimetic mutations have been used to study the impact of phosphorylation on $\mathrm{htt}$ aggregation, and the consensus is that these mutations reduce fibrillization and fibril stability. 37,39,47,50,51,54,72 However, such mutations are imperfect models of phosphorylation. ${ }^{73}$ Recent studies have explored the impact of bona fide phosphorylation within $\mathrm{Nt17}$ on htt aggregation. ${ }^{74,75}$ Actual phosphorylation at $\mathrm{S} 13$ and/or S16 impeded fibrillization and disrupted the a-helix formation in Nt17; S13D and S16D mutations did not strongly inhibit $\alpha$-helix formation. ${ }^{74}$ This inhibited $\alpha$-helix formation strongly reduced the interaction with lipid membranes. Phosphorylation at T3 stabilizes $\alpha$-helix formation. ${ }^{54}$ An additional caveat here is that only single phosphomimetic mutants were investigated. There is crosstalk between different PTM sites within Nt17 with implications for aggregation. Combinations of phosphorylation at 
T3, S13, and S16 have profound impacts on Nt17 structure and htt aggregation. ${ }^{74}$ In addition, there is crosstalk associated between $\mathrm{T} 3$ and $\mathrm{K} 6$, with acetylation at $\mathrm{K} 6$ reversing the inhibitory impact on htt aggregation associated with phosphorylation at T3. ${ }^{54}$ Despite these caveats, there is still value in using phosphomimetic mutations to understand how Nt17 phosphorylation regulates htt aggregation and membrane activity as introduction of phosphomimetic mutations (S13D/S16D) in the context of the fulllength mutant htt improved phenotype in an HD mouse model. ${ }^{39}$ This suggests that, while phosphomimetic mutations may not provide exact models of the impact of phosphorylation of htt in HD, these mutations do modify phenotype and biophysical characterization can provide insights into potential toxic mechanisms. As such, understanding the biophysical impact of phosphomimetic mutations on htt aggregation and membrane activity may provide mechanistic detail concerning htt related toxicity.

The differences in aggregation associated with the different lipid systems underscores the complexity of htt/lipid interactions. Experiments and simulations indicate that the structure and orientation of $\mathrm{Nt} 17$ on membranes is an amphipathic $\alpha$ helix that sequesters nonpolar residues toward the membrane hydrophobic core. ${ }^{76,77}$ Electrostatic interactions between Nt17 and lipids influences the affinity of Nt17 for phospholipid bilayers, ${ }^{78,79}$ and a phosphomimetic double mutation (S13D and S16D) reduced the binding affinity of htt for POPC/POPS mixed lipid systems, requiring 15 times the lipid content to reach saturation and suggesting an introduced electrostatic repulsion. ${ }^{26}$ Similar to what was observed here for aggregation in the presence of POPC or TBLE vesicles, the double mutation (S13D and S16D) suppresses htt aggregation with POPC/POPS vesicles. ${ }^{26}$ While we generally observed a large decrease in the 
membrane binding of htt with phosphomimetic mutations with TBLE based on PDA assays, changes in membrane binding associated with phosphomimetic mutations observed here with POPC and POPG vesicles were subtle and often not significant. Beyond the use of different lipid systems between these studies, a potential explanation is that the single mutations used here only neutralized the net charge of Nt17 while the double mutation resulted in a net negative charge. Here, POPG enhanced aggregation even when the net charge of Nt17 was neutralized with little impact on htt membrane affinity. Taking into account that Nt17 contains numerous residues (T3, K6, K9, S13, $\mathrm{K} 15$, and S16) that can undergo charge altering PTMs ${ }^{34}$ and that crosstalk between these residues influence their modification and aggregation, ${ }^{54,74}$ modifying charge via combinations of PTMs in Nt17 can serve a potential role for targeting specific membranes of differing phospholipid composition. In this regard, phosphorylation and acetylation modifies the localization of huntingtin throughout cells. $17,38,39,70,80,81$

The direct observation, via in situ AFM, that the introduction of phosphomimetic mutations in htt-exon1 altered the morphology of aggregates on supported TBLE bilayers further demonstrates the complex role interfaces play in these processes. While determining all the factors influencing aggregation in vivo remains difficult, a key component of the cellular environment is the presence of interfaces associated with membranes. Solid/liquid interfaces profoundly impact the aggregation process (both kinetically and morphologically) of numerous amyloid-forming proteins. ${ }^{82}$ For example, $\beta$ amyloid peptide $(A \beta)$ displays distinct aggregation pathways on chemically diverse surfaces. On the anionic surface of mica, $A \beta$ forms small, highly mobile oligomers that coalesce into prefibrillar aggregates. ${ }^{83}$ In contrast, epitaxial effects associated with 
exposure to graphitic surfaces promotes the formation of extended nanoribbons with distinct orientations on the surface. ${ }^{83}$ The introduction of point mutations clustered around the central hydrophobic core of $A \beta$ (E22G Arctic mutation, E22K Italian mutation, D23N lowa mutation, and $A 21 G$ Flemish mutation) impact the rate of $A \beta$ aggregation in bulk solution, but do not alter the morphology of aggregates once formed. However, these mutations have a pronounced impact on aggregate morphology when aggregation was directly observed on either mica ${ }^{84}$ or supported TBLE bilayers, ${ }^{64}$ demonstrating that a change in a single amino acid strongly influences protein/surface interactions. Regarding physiological relevance, cellular and subcellular membranes provide important quasisolid/liquid interfaces within cells. The ability of the lipid membranes to influence htt aggregation has been well established, with different lipid systems often having a distinct impact. ${ }^{22,32,33,85}$ Like the afore mentioned point mutations in $A \beta$, phosphomimetic mutations in htt-exon1 promoted distinct aggregate morphology on TBLE bilayers. This adds another layer of complexity when unraveling the heterogeneous mixture of aggregates forming in the cellular environment and assigning toxic function to specific aggregate forms. Htt/lipid interactions and factors modifying these interactions can have a pronounced impact therapeutic strategy aimed at modifying the aggregation process. In this regard, the presence of lipid membranes abolished the ability of the small molecule curcumin from inhibiting htt fibrillization. ${ }^{85}$

Understanding the interplay between htt and membranous surfaces is critical for understanding a variety of associated toxic mechanisms. A number of biophysical functions are associated with lipid binding amphipathic $\alpha$-helices ${ }^{86}$ such as Nt17. These include detecting and binding highly curved lipid membranes, ${ }^{87,88}$ and Nt17 
demonstrates this propensity. ${ }^{26,31}$ Amphipathic $\alpha$-helixes bind membranes weakly and are regulated by a variety of PTMs. Phosphorylation in Nt17 appears to perform such a regulatory role in cellular trafficking of $h t t, 37,39,71,74$ suggesting that phosphorylation plays a role in the selectivity of htt binding to a variety of subcellular membranes of varying lipid composition. The impact of phosphorylation to stabilize or destabilize $\alpha$-helix formation is one contributing factor in altering htt's affinity for membranes, ${ }^{74}$ but selectivity likely works in conjunction with membrane composition. Lipid composition determines the physicochemical properties of membranes that influence amyloid/membrane interactions. ${ }^{82}$ Beyond electrostatic considerations, the aforementioned propensity of Nt17 for curved membranes suggest that selectivity of httexon1 for different membranes may be related to lateral membrane pressure and transient packing defects, both of which are readily influenced by curvature. ${ }^{89}$ The introduction of phosphomimetic mutations, especially $\mathrm{T} 3$ and $\mathrm{S} 13$, enhanced the affinity of htt for TBLE, yet TBLE strongly inhibited fibril formation while stabilizing oligomer size. The complexity of TBLE, which is comprised of a complex, physiologically relevant ratio of zwitterionic and negatively charged lipids (9.6\% phosphatidylcholine, $16.7 \%$ phosphatidylethanolamine, $1.6 \%$ phosphatidylinositol, $10.6 \%$ phosphatidylserine, $2.8 \%$ phosphatidic acid, etc.), provides a varied surface that provides an array of transient defects to interact with Nt17. TBLE also contains $\sim 20-25 \%$ cholesterol, which significantly alters htt aggregation on membranes. ${ }^{22}$ In this regard, brain extracts promote $\alpha$-helical structure within Nt17. ${ }^{27}$ The affinity of htt for POPC and POPG was less impacted by phosphorylation, suggesting that tuning of membrane affinity of httexon1 by phosphorylation is more sensitive to complex lipid membranes. 


\subsection{References}

(1) Macdonald, M. (1993) A novel gene containing a trinucleotide repeat that is expanded and unstable on Huntington's disease chromosomes. Cell 72, 971-983.

(2) Wetzel, R. (2012) Physical chemistry of polyglutamine: Intriguing tales of a monotonous sequence. J Mol Biol 421, 466-490.

(3) Legleiter, J., Mitchell, E., Lotz, G. P., Sapp, E., Ng, C., DiFiglia, M., Thompson, L. M., and Muchowski, P. J. (2010) Mutant Huntingtin Fragments Form Oligomers in a Polyglutamine Length-dependent Manner in Vitro and in Vivo. J Biol Chem 285, 1477714790 .

(4) Adegbuyiro, A., Sedighi, F., Pilkington, A. W., Groover, S., and Legleiter, J. (2017) Proteins Containing Expanded Polyglutamine Tracts and Neurodegenerative Disease. Biochemistry 56, 1199-1217.

(5) Lajoie, P., and Snapp, E. L. (2010) Formation and Toxicity of Soluble Polyglutamine Oligomers in Living Cells. PLoS ONE (Chirico, G., Ed.) 5, e15245.

(6) Nucifora, L. G., Burke, K. A., Feng, X., Arbez, N., Zhu, S., Miller, J., Yang, G., Ratovitski, T., Delannoy, M., Muchowski, P. J., Finkbeiner, S., Legleiter, J., Ross, C. A., and Poirier, M. A. (2012) Identification of Novel Potentially Toxic Oligomers Formed in Vitro from Mammalian-derived Expanded huntingtin Exon-1 Protein. J Biol Chem 287, 16017-16028.

(7) Kim, Y. E., Hosp, F., Frottin, F., Ge, H., Mann, M., Hayer-Hartl, M., and Hartl, F. U. (2016) Soluble Oligomers of PolyQ-Expanded Huntingtin Target a Multiplicity of Key Cellular Factors. Mol Cell 63, 951-964.

(8) Drombosky, K. W., Rode, S., Kodali, R., Jacob, T. C., Palladino, M. J., and Wetzel, R. (2018) Mutational analysis implicates the amyloid fibril as the toxic entity in Huntington's disease. Neurobiol Dis 120, 126-138.

(9) Pieri, L., Madiona, K., Bousset, L., and Melki, R. (2012) Fibrillar a-Synuclein and Huntingtin Exon 1 Assemblies Are Toxic to the Cells. Biophys J 102, 2894-2905. (10) Bäuerlein, F. J. B., Saha, I., Mishra, A., Kalemanov, M., Martínez-Sánchez, A., Klein, R., Dudanova, I., Hipp, M. S., Hartl, F. U., Baumeister, W., and FernándezBusnadiego, R. (2017) In Situ Architecture and Cellular Interactions of PolyQ Inclusions. Cell 171, 179-187.e10.

(11) Gutekunst, C.-A., Li, S.-H., Yi, H., Mulroy, J. S., Kuemmerle, S., Jones, R., Rye, D., Ferrante, R. J., Hersch, S. M., and Li, X.-J. (1999) Nuclear and Neuropil Aggregates in Huntington's Disease: Relationship to Neuropathology. J Neurosci 19, 2522-2534. (12) Orr, A. L., Li, S., Wang, C.-E., Li, H., Wang, J., Rong, J., Xu, X., Mastroberardino, P. G., Greenamyre, J. T., and Li, X.-J. (2008) N-Terminal Mutant Huntingtin Associates with Mitochondria and Impairs Mitochondrial Trafficking. J Neurosci 28, 2783-2792. (13) Panov, A. V., Gutekunst, C.-A., Leavitt, B. R., Hayden, M. R., Burke, J. R., Strittmatter, W. J., and Greenamyre, J. T. (2002) Early mitochondrial calcium defects in Huntington's disease are a direct effect of polyglutamines. Nat Neurosci 5, 731-736. (14) Shirendeb, U., Reddy, A. P., Manczak, M., Calkins, M. J., Mao, P., Tagle, D. A., and Hemachandra Reddy, P. (2011) Abnormal mitochondrial dynamics, mitochondrial loss and mutant huntingtin oligomers in Huntington's disease: implications for selective neuronal damage. Hum Mol Genet 20, 1438-1455. 
(15) Liu, K.-Y., Shyu, Y.-C., Barbaro, B. A., Lin, Y.-T., Chern, Y., Thompson, L. M., James Shen, C.-K., and Marsh, J. L. (2015) Disruption of the nuclear membrane by perinuclear inclusions of mutant huntingtin causes cell-cycle re-entry and striatal cell death in mouse and cell models of Huntington's disease. Hum Mol Genet 24, 16021616.

(16) Ueda, M., Li, S., Itoh, M., Wang, M., Hayakawa, M., Islam, S., Tana, Nakagawa, K., Chen, H., and Nakagawa, T. (2016) Expanded polyglutamine embedded in the endoplasmic reticulum causes membrane distortion and coincides with Bax insertion. Biochem Biophys Res Co 474, 259-263.

(17) Rockabrand, E., Slepko, N., Pantalone, A., Nukala, V. N., Kazantsev, A., Marsh, J. L., Sullivan, P. G., Steffan, J. S., Sensi, S. L., and Thompson, L. M. (2007) The first 17 amino acids of Huntingtin modulate its sub-cellular localization, aggregation and effects on calcium homeostasis. Hum Mol Genet 16, 61-77.

(18) Qin, Z.-H. (2004) Huntingtin Bodies Sequester Vesicle-Associated Proteins by a Polyproline-Dependent Interaction. J Neurosci 24, 269-281.

(19) Kegel, K. B., Sapp, E., Yoder, J., Cuiffo, B., Sobin, L., Kim, Y. J., Qin, Z.-H., Hayden, M. R., Aronin, N., Scott, D. L., Isenberg, G., Goldmann, W. H., and DiFiglia, M. (2005) Huntingtin Associates with Acidic Phospholipids at the Plasma Membrane. J Biol Chem 280, 36464-36473.

(20) Kegel, K. B., Kim, M., Sapp, E., McIntyre, C., Castaño, J. G., Aronin, N., and DiFiglia, M. (2000) Huntingtin Expression Stimulates Endosomal-Lysosomal Activity, Endosome Tubulation, and Autophagy. J Neurosci 20, 7268-7278.

(21) Burke, K. A., Kauffman, K. J., Umbaugh, C. S., Frey, S. L., and Legleiter, J. (2013) The Interaction of Polyglutamine Peptides with Lipid Membranes Is Regulated by Flanking Sequences Associated with Huntingtin. J Biol Chem 288, 14993-15005. (22) Gao, X., Campbell, W. A., Chaibva, M., Jain, P., Leslie, A. E., Frey, S. L., and Legleiter, J. (2016) Cholesterol Modifies Huntingtin Binding to, Disruption of, and Aggregation on Lipid Membranes. Biochemistry 55, 92-102.

(23) Chaibva, M., Gao, X., Jain, P., Campbell, W. A., Frey, S. L., and Legleiter, J. (2018) Sphingomyelin and GM1 Influence Huntingtin Binding to, Disruption of, and Aggregation on Lipid Membranes. ACS Omega 3, 273-285.

(24) Burke, K. A., Yates, E. A., and Legleiter, J. (2013) Amyloid-Forming Proteins Alter the Local Mechanical Properties of Lipid Membranes. Biochemistry 52, 808-817. (25) Choo, Y. S., Johnson, G. V. W., MacDonald, M., Detloff, P. J., and Lesort, M. (2004) Mutant huntingtin directly increases susceptibility of mitochondria to the calciuminduced permeability transition and cytochrome c release. Hum Mol Genet 13, 14071420.

(26) Tao, M., Pandey, N. K., Barnes, R., Han, S., and Langen, R. (2019) Structure of Membrane-Bound Huntingtin Exon 1 Reveals Membrane Interaction and Aggregation Mechanisms. Structure 27, 1570-1580.e4.

(27) Levy, G. R., Shen, K., Gavrilov, Y., Smith, P. E. S., Levy, Y., Chan, R., Frydman, J., and Frydman, L. (2019) Huntingtin's N-Terminus Rearrangements in the Presence of Membranes: A Joint Spectroscopic and Computational Perspective. ACS Chem Neurosci 10, 472-481.

(28) Arndt, J. R., Chaibva, M., Beasley, M., Kiani Karanji, A., Ghassabi Kondalaji, S., Khakinejad, M., Sarver, O., Legleiter, J., and Valentine, S. J. (2020) Nucleation 
Inhibition of Huntingtin Protein (htt) by Polyproline PPII Helices: A Potential Interaction with the N-Terminal $\alpha$-Helical Region of Htt. Biochemistry 59, 436-449.

(29) Ceccon, A., Schmidt, T., Tugarinov, V., Kotler, S. A., Schwieters, C. D., and Clore, G. M. (2018) Interaction of Huntingtin Exon-1 Peptides with Lipid-Based Micellar Nanoparticles Probed by Solution NMR and Q-Band Pulsed EPR. J Am Chem Soc 140, 6199-6202.

(30) Chaibva, M., Arndt, J. R., Valentine, S. J., and Legleiter, J. (2015) Acetylation Regulates the Interaction of Huntingtin with Lipid Membranes: Implications for Huntington Disease. Biophys J 108, 254a.

(31) Chaibva, M., Burke, K. A., and Legleiter, J. (2014) Curvature Enhances Binding and Aggregation of Huntingtin at Lipid Membranes. Biochemistry 53, 2355-2365.

(32) Pandey, N. K., Isas, J. M., Rawat, A., Lee, R. V., Langen, J., Pandey, P., and Langen, R. (2018) The 17-residue-long $\mathrm{N}$ terminus in huntingtin controls stepwise aggregation in solution and on membranes via different mechanisms. J Biol Chem 293, 2597-2605.

(33) Burke, K. A., Hensal, K. M., Umbaugh, C. S., Chaibva, M., and Legleiter, J. (2013) Huntingtin disrupts lipid bilayers in a polyQ-length dependent manner. BBA -

Biomembranes 1828, 1953-1961.

(34) Arndt, J. R., Chaibva, M., and Legleiter, J. (2015) The emerging role of the first 17 amino acids of huntingtin in Huntington's disease. Biomol Concepts 6, 33-46.

(35) Jayaraman, M., Kodali, R., Sahoo, B., Thakur, A. K., Mayasundari, A., Mishra, R., Peterson, C. B., and Wetzel, R. (2012) Slow Amyloid Nucleation via a-Helix-Rich Oligomeric Intermediates in Short Polyglutamine-Containing Huntingtin Fragments. $J$ Mol Biol 415, 881-899.

(36) Mishra, R., Jayaraman, M., Roland, B. P., Landrum, E., Fullam, T., Kodali, R., Thakur, A. K., Arduini, I., and Wetzel, R. (2012) Inhibiting nucleation of amyloid structure in a huntingtin fragment by targeting a-helix rich oligomeric intermediates. $J$ Mol Biol 415, 900-917.

(37) Aiken, C. T., Steffan, J. S., Guerrero, C. M., Khashwji, H., Lukacsovich, T., Simmons, D., Purcell, J. M., Menhaji, K., Zhu, Y.-Z., Green, K., LaFerla, F., Huang, L., Thompson, L. M., and Marsh, J. L. (2009) Phosphorylation of Threonine 3. J Biol Chem 284, 29427-29436.

(38) Thompson, L. M., Aiken, C. T., Kaltenbach, L. S., Agrawal, N., Illes, K., Khoshnan, A., Martinez-Vincente, M., Arrasate, M., O'Rourke, J. G., Khashwji, H., Lukacsovich, T., Zhu, Y.-Z., Lau, A. L., Massey, A., Hayden, M. R., Zeitlin, S. O., Finkbeiner, S., Green, K. N., LaFerla, F. M., Bates, G., Huang, L., Patterson, P. H., Lo, D. C., Cuervo, A. M., Marsh, J. L., and Steffan, J. S. (2009) IKK phosphorylates Huntingtin and targets it for degradation by the proteasome and lysosome. J Cell Biol 187, 1083-1099.

(39) Gu, X., Greiner, E. R., Mishra, R., Kodali, R., Osmand, A., Finkbeiner, S., Steffan, J. S., Thompson, L. M., Wetzel, R., and Yang, X. W. (2009) Serines 13 and 16 Are Critical Determinants of Full-length Human Mutant Huntingtin-Induced Disease Pathogenesis in HD Mice. Neuron 64, 828-840.

(40) Di Pardo, A., Maglione, V., Alpaugh, M., Horkey, M., Atwal, R. S., Sassone, J., Ciammola, A., Steffan, J. S., Fouad, K., Truant, R., and Sipione, S. (2012) Ganglioside GM1 induces phosphorylation of mutant huntingtin and restores normal motor behavior in Huntington disease mice. Proc Natl Acad Sci USA 109, 3528-3533. 
(41) Mitomi, Y., Nomura, T., Kurosawa, M., Nukina, N., and Furukawa, Y. (2012) Postaggregation Oxidation of Mutant Huntingtin Controls the Interactions between Aggregates. J Biol Chem 287, 34764-34775.

(42) DiGiovanni, L. F., Mocle, A. J., Xia, J., and Truant, R. (2016) Huntingtin N17 domain is a reactive oxygen species sensor regulating huntingtin phosphorylation and localization. Hum Mol Genet 25, 3937-3945.

(43) Cong, X., Held, J. M., DeGiacomo, F., Bonner, A., Chen, J. M., Schilling, B., Czerwieniec, G. A., Gibson, B. W., and Ellerby, L. M. (2011) Mass Spectrometric Identification of Novel Lysine Acetylation Sites in Huntingtin. Mol Cell Proteomics 10. (44) Díaz-Hernández, M., Valera, A. G., Morán, M. A., Gómez-Ramos, P., AlvarezCastelao, B., Castaño, J. G., Hernández, F., and Lucas, J. J. (2006) Inhibition of 26S proteasome activity by huntingtin filaments but not inclusion bodies isolated from mouse and human brain. $J$ Neurochem 98, 1585-1596.

(45) Steffan, J. S., Agrawal, N., Pallos, J., Rockabrand, E., Trotman, L. C., Slepko, N., Illes, K., Lukacsovich, T., Zhu, Y.-Z., Cattaneo, E., Pandolfi, P. P., Thompson, L. M., and Marsh, J. L. (2004) SUMO Modification of Huntingtin and Huntington's Disease Pathology. Science 304, 100-104.

(46) Pennuto, M., Palazzolo, I., and Poletti, A. (2009) Post-translational modifications of expanded polyglutamine proteins: impact on neurotoxicity. Hum Mol Genet 18, R40R47.

(47) Mishra, R., Hoop, C. L., Kodali, R., Sahoo, B., van der Wel, P. C. A., and Wetzel, R. (2012) Serine phosphorylation suppresses huntingtin amyloid accumulation by altering protein aggregation properties. J Mol Biol 424, 1-14.

(48) Zheng, Z., Li, A., Holmes, B. B., Marasa, J. C., and Diamond, M. I. (2013) An Nterminal Nuclear Export Signal Regulates Trafficking and Aggregation of Huntingtin (Htt) Protein Exon 1. J Biol Chem 288, 6063-6071.

(49) Maiuri, T., Woloshansky, T., Xia, J., and Truant, R. (2013) The huntingtin N17 domain is a multifunctional CRM1 and Ran-dependent nuclear and cilial export signal. Hum Mol Genet 22, 1383-1394.

(50) Cariulo, C., Verani, M., Martufi, P., Ingenito, R., Finotto, M., Deguire, S. M., Lavery, D. J., Toledo-Sherman, L., Lee, R., Doherty, E. M., Vogt, T. F., Dominguez, C., Lashuel, H. A., Petricca, L., and Caricasole, A. (2020) Ultrasensitive quantitative measurement of huntingtin phosphorylation at residue S13. Biochem Biophys Res Co 521, 549-554.

(51) Herrera, F., Branco-Santos, J., and PhD, T. F. O. (2019) Threonine 3 regulates Serine 13/16 phosphorylation in the huntingtin exon 1. Matters Select 5, e201905000005.

(52) Arndt, J. R., Kondalaji, S. G., Maurer, M. M., Parker, A., Legleiter, J., and Valentine, S. J. (2015) Huntingtin N-terminal monomeric and multimeric structures destabilized by covalent modification of heteroatomic residues. Biochemistry 54, 42854296.

(53) Ansaloni, A., Wang, Z.-M., Jeong, J. S., Ruggeri, F. S., Dietler, G., and Lashuel, H. A. (2014) One-Pot Semisynthesis of Exon 1 of the Huntingtin Protein: New Tools for Elucidating the Role of Posttranslational Modifications in the Pathogenesis of Huntington's Disease. Angew Chem Intl Ed 53, 1928-1933.

(54) Chiki, A., DeGuire, S. M., Ruggeri, F. S., Sanfelice, D., Ansaloni, A., Wang, Z.-M., Cendrowska, U., Burai, R., Vieweg, S., Pastore, A., Dietler, G., and Lashuel, H. A. 
(2017) Mutant Exon1 Huntingtin Aggregation is Regulated by T3 PhosphorylationInduced Structural Changes and Crosstalk between T3 Phosphorylation and Acetylation at K6. Angew Chem Intl Ed 56, 5202-5207.

(55) Branco-Santos, J., Herrera, F., Poças, G. M., Pires-Afonso, Y., Giorgini, F., Domingos, P. M., and Outeiro, T. F. (2017) Protein phosphatase 1 regulates huntingtin exon 1 aggregation and toxicity. Hum Mol Genet 26, 3763-3775.

(56) Muchowski, P. J., Schaffar, G., Sittler, A., Wanker, E. E., Hayer-Hartl, M. K., and Hartl, F. U. (2000) Hsp70 and hsp40 chaperones can inhibit self-assembly of polyglutamine proteins into amyloid-like fibrils. Proc Natl Acad Sci USA 97, 7841-7846. (57) Beasley, M., Stonebraker, A. R., and Legleiter, J. Normalizing Polydiacetylene Colorimetric Assays of Vesicle Binding Across Lipid Systems. Anal Biochem (in press). (58) Zheng, F., Wu, Z., and Chen, Y. (2012) A quantitative method for the measurement of membrane affinity by polydiacetylene-based colorimetric assay. Anal Biochem 420, 171-176.

(59) Sokolovski, M., Sheynis, T., Kolusheva, S., and Jelinek, R. (2008) Membrane interactions and lipid binding of casein oligomers and early aggregates. BBA Biomembranes 1778, 2341-2349.

(60) Burke, K. A., Godbey, J., and Legleiter, J. (2011) Assessing mutant huntingtin fragment and polyglutamine aggregation by atomic force microscopy. Methods 53, 275284.

(61) Burke, K. A., and Legleiter, J. (2013) Atomic force microscopy assays for evaluating polyglutamine aggregation in solution and on surfaces. Methods Mol. Biol. 1017, 21-40.

(62) Yates, E. A., and Legleiter, J. (2014) Preparation Protocols of A $\beta(1-40)$ Promote the Formation of Polymorphic Aggregates and Altered Interactions with Lipid Bilayers. Biochemistry 53, 7038-7050.

(63) Sedighi, F., Adegbuyiro, A., and Legleiter, J. (2020) SUMOylation Prevents Huntingtin Fibrillization and Localization onto Lipid Membranes. ACS Chem Neurosci 11, 328-343.

(64) Pifer, P. M., Yates, E. A., and Legleiter, J. (2011) Point Mutations in A $\beta$ Result in the Formation of Distinct Polymorphic Aggregates in the Presence of Lipid Bilayers.

PLoS ONE (Uversky, V. N., Ed.) 6, e16248.

(65) Yates, E. A., Owens, S. L., Lynch, M. F., Cucco, E. M., Umbaugh, C. S., and Legleiter, J. (2013) Specific Domains of A $\beta$ Facilitate Aggregation on and Association with Lipid Bilayers. J Mol Biol 425, 1915-1933.

(66) Legleiter, J., Fryer, J. D., Holtzman, D. M., and Kowalewski, T. (2011) The Modulating Effect of Mechanical Changes in Lipid Bilayers Caused by ApoE-Containing Lipoproteins on A $\beta$ Induced Membrane Disruption. ACS Chem Neurosci 2, 588-599.

(67) Thakur, A. K., Jayaraman, M., Mishra, R., Thakur, M., Chellgren, V. M., Byeon, I.J., Anjum, D. H., Kodali, R., Creamer, T. P., Conway, J. F., M.Gronenborn, A., and Wetzel, R. (2009) Polyglutamine disruption of the huntingtin exon1 N-terminus triggers a complex aggregation mechanism. Nat Struct Mol Biol 16, 380-389.

(68) Chongtham, A., Bornemann, D. J., Barbaro, B. A., Lukacsovich, T., Agrawal, N., Syed, A., Worthge, S., Purcell, J., Burke, J., Chin, T. M., and Marsh, J. L. (2020) Effects of flanking sequences and cellular context on subcellular behavior and pathology of mutant HTT. Hum Mol Genet 29, 674-688. 
(69) Lin, H.-K., Boatz, J. C., Krabbendam, I. E., Kodali, R., Hou, Z., Wetzel, R., Dolga, A. M., Poirier, M. A., and van der Wel, P. C. A. (2017) Fibril polymorphism affects immobilized non-amyloid flanking domains of huntingtin exon1 rather than its polyglutamine core. Nat Commun 8, 15462.

(70) Atwal, R. S., Xia, J., Pinchev, D., Taylor, J., Epand, R. M., and Truant, R. (2007) Huntingtin has a membrane association signal that can modulate huntingtin aggregation, nuclear entry and toxicity. Hum Mol Genet 16, 2600-2615.

(71) Atwal, R. S., Desmond, C. R., Caron, N., Maiuri, T., Xia, J., Sipione, S., and Truant, R. (2011) Kinase inhibitors modulate huntingtin cell localization and toxicity. Nat Chem Biol 7, 453-460.

(72) Cariulo, C., Azzollini, L., Verani, M., Martufi, P., Boggio, R., Chiki, A., Deguire, S. M., Cherubini, M., Gines, S., Marsh, J. L., Conforti, P., Cattaneo, E., Santimone, I., Squitieri, F., Lashuel, H. A., Petricca, L., and Caricasole, A. (2017) Phosphorylation of huntingtin at residue T3 is decreased in Huntington's disease and modulates mutant huntingtin protein conformation. Proc Natl Acad Sci USA 114, E10809-E10818. (73) Dephoure, N., Gould, K. L., Gygi, S. P., and Kellogg, D. R. (2013) Mapping and analysis of phosphorylation sites: a quick guide for cell biologists. Mol Biol Cell 24, 535542.

(74) DeGuire, S. M., Ruggeri, F. S., Fares, M.-B., Chiki, A., Cendrowska, U., Dietler, G., and Lashuel, H. A. (2018) N-terminal Huntingtin (Htt) phosphorylation is a molecular switch regulating $\mathrm{Htt}$ aggregation, helical conformation, internalization, and nuclear targeting. J Biol Chem 293, 18540-18558.

(75) Daldin, M., Fodale, V., Cariulo, C., Azzollini, L., Verani, M., Martufi, P., Spiezia, M. C., Deguire, S. M., Cherubini, M., Macdonald, D., Weiss, A., Bresciani, A., Vonsattel, J.P. G., Petricca, L., Marsh, J. L., Gines, S., Santimone, I., Marano, M., Lashuel, H. A., Squitieri, F., and Caricasole, A. (2017) Polyglutamine expansion affects huntingtin conformation in multiple Huntington's disease models. Sci Rep 7.

(76) Michalek, M., Salnikov, E. S., and Bechinger, B. (2013) Structure and Topology of the Huntingtin 1-17 Membrane Anchor by a Combined Solution and Solid-State NMR Approach. Biophys J 105, 699-710.

(77) Michalek, M., Salnikov, E. S., Werten, S., and Bechinger, B. (2013) Membrane Interactions of the Amphipathic Amino Terminus of Huntingtin. Biochemistry 52, 847858.

(78) Nagarajan, A., Jawahery, S., and Matysiak, S. (2014) The Effects of Flanking Sequences in the Interaction of Polyglutamine Peptides with a Membrane Bilayer. $J$ Phys Chem B 118, 6368-6379.

(79) Côté, S., Binette, V., Salnikov, E. S., Bechinger, B., and Mousseau, N. (2015) Probing the Huntingtin 1-17 Membrane Anchor on a Phospholipid Bilayer by Using AllAtom Simulations. Biophys J 108, 1187-1198.

(80) Ehrnhoefer, D. E., Sutton, L., and Hayden, M. R. (2011) Small changes, big impact: Posttranslational modifications and function of huntingtin in Huntington disease. Neuroscientist 17, 475-492.

(81) Saudou, F., and Humbert, S. (2016) The Biology of Huntingtin. Neuron 89, 910926. 
(82) Burke, K. A., Yates, E. A., and Legleiter, J. (2013) Biophysical Insights into How Surfaces, Including Lipid Membranes, Modulate Protein Aggregation Related to Neurodegeneration. Front Neurol 4.

(83) Kowalewski, T., and Holtzman, D. M. (1999) In situ atomic force microscopy study of Alzheimer's -amyloid peptide on different substrates: New insights into mechanism of -sheet formation. Proc Natl Acad Sci 96, 3688-3693.

(84) Yates, E. A., Cucco, E. M., and Legleiter, J. (2011) Point Mutations in A $\beta$ Induce Polymorphic Aggregates at Liquid/Solid Interfaces. ACS Chem Neurosci 2, 294-307. (85) Beasley, M., Stonebraker, A. R., Hasan, I., Kapp, K. L., Liang, B. J., Agarwal, G., Groover, S., Sedighi, F., and Legleiter, J. (2019) Lipid Membranes Influence the Ability of Small Molecules To Inhibit Huntingtin Fibrillization. Biochemistry 58, 4361-4373.

(86) Drin, G., and Antonny, B. (2010) Amphipathic helices and membrane curvature. FEBS Letters 584, 1840-1847.

(87) Cui, H., Lyman, E., and Voth, G. A. (2011) Mechanism of Membrane Curvature Sensing by Amphipathic Helix Containing Proteins. Biophys J 100, 1271-1279.

(88) Georgieva, E. R., Xiao, S., Borbat, P. P., Freed, J. H., and Eliezer, D. (2014) Tau Binds to Lipid Membrane Surfaces via Short Amphipathic Helices Located in Its Microtubule-Binding Repeats. Biophys J 107, 1441-1452.

(89) Davidson, W. S., Jonas, A., Clayton, D. F., and George, J. M. Stabilization of $\alpha-$ Synuclein Secondary Structure upon Binding to Synthetic Membranes. J Biol Chem 273(16), 9443-9. 


\title{
3. Macromolecular Crowding Alters Huntingtin Aggregation at Interfaces
}

\begin{abstract}
Huntington's disease (HD) is a fatal neurodegenerative disease caused by an extended polyglutamine (polyQ) domain within the first exon of the huntingtin protein (htt). PolyQ expansion directly invokes the formation of a heterogenous mixture toxic htt aggregates, including fibrils and oligomers. While htt is a cytosolic protein, it also associates with numerous organelle surfaces within the cell, leading to altered organelle morphology and dysfunction. Here, the impact of macromolecular crowding on htt aggregation in bulk solution and at solid/liquid or membrane/liquid interfaces was investigated.

Dextran, ficoll, and polyethylene glycol (PEG) were used as crowding agents. In bulk solution, crowding enhanced the heterogeneity of non-fibrillar aggregate species formed in a crowder dependent manner. Crowding agents interfered with the deposition and formation of htt fibrils on mica. Crowding in the aqueous phase enhanced deposition of htt aggregates onto supported total brain lipid extract (TBLE) supported bilayers, leading to distinct morphological alteration of the bilayer surface. Collectively, these observations point to the complexity of htt aggregation at interfaces and that crowding in the aqueous phase profoundly influences this process.
\end{abstract}

\subsection{Introduction}

Deposition of aggregated, proteinaceous material as plaques and inclusions is a hallmark of several neurodegenerative diseases, i.e. Parkinson's disease (PD), Alzheimer's disease (AD), and Huntington's disease (HD). These deposits are 
predominately comprised of amyloid, characterized by a fibrillar morphology and cross $\beta$-sheet structure. Of these diseases, HD is caused by a mutation in the huntingtin gene that encodes for an abnormal expansion (greater than $\sim 35$ repeats) of a polyglutamine (polyQ) tract in the first exon of the huntingtin protein (htt). ${ }^{1}$ PolyQ expansion correlates with htt aggregation, which is a multi-pathway, complex process involving a variety of aggregate species including oligomers, amorphous aggregates, fibrils, and annular structures. ${ }^{2-4}$ Due to the variety of aggregates possible, htt aggregation results in heterogeneous mixtures of aggregates, making it difficult to elucidate relationships between structure and toxic gain of function. Fibrils, ${ }^{5,6}$ oligomers, ${ }^{7-9}$ and inclusions ${ }^{10}$ are all implicated as potential toxic entities. Due to this, it is imperative to understand how exogenous factors within the cytosolic environment influence aggregation.

While aggregation is a heterogeneous process, the complexity of the intracellular environment can have a pronounced impact on htt aggregation. The cytosolic environment of cells is extremely crowded with proteins and other large macromolecules. Depending on location and cell type, macromolecular concentrations within cells can reach up to $400 \mathrm{mg} / \mathrm{mL}$ and up to $40 \%$ of the available volume is occupied by macromolecules, ${ }^{11-14}$ and such crowded environments influence protein behavior. ${ }^{15}$ For example, macromolecular crowding agents sterically reduce configurational entropy in proteins via an excluded volume mechanism. ${ }^{16}$ In addition, proteins nonspecifically interact with crowding agents via van der Waals forces, hydrophilic/hydrophobic effects, and electrostatics. ${ }^{17}$ These interactions further influence protein conformation. As such, the physicochemical properties of the crowder molecules profoundly influence proteins in numerous ways with implications for amyloid 
formation. ${ }^{18}$ Amyloid formation in a crowded environment can differ by orders of magnitude from that observed in bulk solution. ${ }^{16,19}$ The impact of crowders on different amyloid-forming proteins varies greatly depending on factors such as crowder concentration, and the extent of interaction between the crowding agent and the aggregating protein. For example, $\alpha$-synuclein aggregation is accelerated by numerous crowders, i.e. polyethylene glycol (PEG), dextran, ficoll, lysozyme, and bovine serum albumin; however, larger macromolecular crowders promoted aggregation faster than smaller crowders. ${ }^{20}$ On the other hand, crowders can stabilize multimeric protein states, suppressing nucleation of fibrillization. ${ }^{21}$ In terms of chemical interactions, the extent of saccharide based crowders interaction with $A \beta$ determined whether crowding promoted or inhibited fibril formation. ${ }^{22}$

Beyond crowded environments, the presence of liquid/surface interfaces alter amyloid formation. ${ }^{23}$ Solid surfaces, ${ }^{24,25}$ including mica, graphite, gold, and Teflon, induce conformational changes in proteins, which alter aggregation of a variety of amyloid forming proteins. ${ }^{26-30}$ Surfaces of particular physiological relevance are lipid membranes, which also exert influence on protein aggregation. ${ }^{23}$ In this regard, htt is highly associated with membranous surfaces within cells, and these interactions further modify aggregation. For example, htt and a variety of its aggregated forms localize to the ER, mitochondria, nuclear envelope, tubulovesicles, endosomes, lysosomes, and synaptic vesicles. ${ }^{31-35}$ PolyQ expansion enhances htt/phospholipid interactions. ${ }^{36-38} \mathrm{Htt}$ aggregation disrupts lipid membranes, leading to organelle dysfunction. ${ }^{30,39-41}$ Brain lipids and membranous structures accumulate in htt aggregates, ${ }^{39,42-44}$ suggesting a role of lipids in the aggregation process. Indeed, the impact of a variety of membranes, 
ranging from total brain lipid extracts (TBLE) to pure lipid systems, alter the rate of aggregation and the resulting aggregate species observed. ${ }^{30,45-48} \mathrm{Htt}-\mathrm{exon} 1$ fibril formation is enhanced in the presence of POPC/POPS $45,49,50$ mixed vesicles and pure POPC, POPG, or POPS vesicles. ${ }^{51}$ On the other hand, brain lipid extracts reduce fibrillization, $30,38,52$ and the addition of exogenous lipid components, i.e. cholesterol, sphingomyelin, and GM1,47,48 to these extracts further influence aggregation.

Here, the impact of macromolecular crowding on htt aggregation was investigated. Three different crowders (Dextran, Ficoll, and polyethylene glycol (PEG)) were used. In particular, the impact of macromolecular crowding in the aqueous phase on htt aggregation and solid/liquid or membrane/liquid interfaces was determined. Continuous imaging using in situ atomic force microscopy (AFM), allowing for the tracking of individual aggregates on surfaces, monitored aggregation at these interfaces. Despite the different macromolecular crowders being considered chemically inert, ${ }^{53,54}$ each exerted differing influence on htt aggregation in bulk solution with Ficoll promoting a unique aggregate morphology. While ThT assays suggested that fibrils still formed, crowders interfered with fibril deposition onto mica. When monitoring aggregation occurring directly on mica, a variety of oligomers and fibrils were observed, but the extent of surface aggregation was impeded by crowding in the aqueous phase, with PEG completely blocking the observation of fibrils. When supported total brain lipid extract (TBLE) bilayers were exposed to htt, crowding in the aqueous phase by all three macromolecules enhanced htt deposition on the bilayer, resulting in increased membrane morphological disruption. Here, PEG was associated with the most aggressive extent of membrane damage. 


\subsection{Materials and Methods}

3.2.1 Expression, Purification of (GST)-htt-exon1 Fusion Protein. A (GST)-httexon1(46Q) fusion protein was purified as described previously. ${ }^{55}$ In short, GST-httexon1 was expressed in $E$. Coli. Resulting cells were lysed with lysozyme $(0.5 \mathrm{mg} / \mathrm{mL})$ and probe sonication. The target fusion protein was purified from these lysates using a GST affinity column and liquid chromatography (BioLogic LPLC, Bio Rad). The purity of the target protein in relevant Fractions was assessed by SDS-PAGE. Protein concentration was determined by a Bradford assay. Prior to each experiment, preexisting aggregates were removed via high speed centrifugation $\left(20,000 \times \mathrm{g}\right.$ at $4^{\circ} \mathrm{C}$ for 30 minutes). Factor Xa protease (New England Bio Labs) was used to cleave GST and initiate aggregation. All experiments were performed in Tris buffer $(50 \mathrm{mM}$ Tris- $\mathrm{HCl}, \mathrm{pH}$ 7, $150 \mathrm{mM} \mathrm{NaCl})$.

3.2.2 Macromolecular Crowder Preparation. Stock solutions $(800 \mathrm{mg} / \mathrm{mL})$ of Ficoll 400, Dextran 20,000, and Polyethylene Glycol 20,000 (PEG) of macromolecular crowders were made in Tris buffer heated to $60^{\circ} \mathrm{C}$, resulting in viscous solutions. These solutions were incubated in an orbital thermomixer at $60^{\circ} \mathrm{C}$ and $1400 \mathrm{rpm}$, followed by bath sonication at $60^{\circ} \mathrm{C}$ for $1 \mathrm{~h}$. These stock solutions were diluted to reach the final desired crowder concentrations in various experiments.

3.2.3 Peptide Preparation. A custom, synthetic peptide based on htt-exon1,

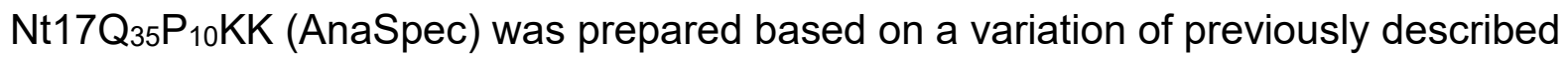
protocols. ${ }^{56}$ Briefly, peptide was dissolved in a 1:1 mixture of hexafluoroisoproponal (Acros Organics) and trifluoroacetic acid (Acros Organics) for $12 \mathrm{~h}$ without agitation. Excess solvent was evaporated off using a vacufuge. The resulting films were dissolved 
in DMSO at a concentration of $2000 \mu \mathrm{M}$. These DMSO stock solutions were diluted into Tris buffer (20 $\mu \mathrm{M}, 1 \% \mathrm{DMSO})$, and this solution was injected directly into the AFM fluid cell (final concentration of $10 \mu \mathrm{M}, 0.5 \% \mathrm{DMSO}$ ).

3.2.4 Lipid Vesicle Preparation. Total brain lipid extract (TBLE; Avanti Polar Lipids) was hydrated in Tris buffer with vortex mixing at $60^{\circ} \mathrm{C}$ until all lipid was suspended in solution. Vesicle formation was promoted by 10 freeze/thaw cycles using liquid nitrogen, followed by bath sonication for 1 hour at room temperature. The vesicle size distribution was analyzed by dynamic light scattering (NanoBrook Omni particle size analyzer, Brookhaven).

3.2.5 Thioflavin T (ThT) Aggregation Assay. Htt-exon1(46Q) was diluted to a final concentration of $10 \mu \mathrm{M}$ in the presence of $125 \mu \mathrm{M}$ ThT (Sigma-Aldrich, St. Louis, MO). Assays were performed in a 96-well plate format ( $100 \mu \mathrm{L}$ per well) using a microplate reader (Molecular Devices, SpectraMax M2). All assays were run at $37^{\circ} \mathrm{C}$, and readings were taken every 5 min with $440 \mathrm{~nm}$ excitation and $484 \mathrm{~nm}$ emission. All conditions were run in triplicate with a minimum of three independent experiments performed. In ThT assays with lipids, a 10:1 lipid:protein ratio was used.

3.2.6 Polydiacetylene (PDA) Vesicle Binding Assay. 10,12-tricosadiynoic acid (GFS Chemicals, Columbus, $\mathrm{OH}$ ) and TBLE was dissolved at a 2:3 molar ratio in a solution of 1:4 ethanol/chloroform. Excess solution was evaporated off under a stream of $\mathrm{N}_{2}$, and the resulting film was resuspended in Tris buffer at $70^{\circ} \mathrm{C}$. Probe sonication (10-minute intervals at $100 \mathrm{~W}$ ) using a sonic dismembrator (FisherSci) was performed to achieve an opaque solution. This solution was stored at $4^{\circ} \mathrm{C}$ overnight to allow self-assembly into vesicles. Polymerization of the PDA was initiated by irradiation at $254 \mathrm{~nm}$ for 10 
minutes at room temperature with stirring, resulting in a blue color. The assay was performed in a 96-well plate format using a microplate reader (Molecular Devices, SpectraMax M2). The percent colorimetric response ( $\% \mathrm{CR}$ ) was determined every 5 min by measuring the blue absorbance $(640 \mathrm{~nm}$, Ablue) and the red absorbance (500 $\left.\mathrm{nm}, \mathrm{A}_{\mathrm{red}}\right)$ using the following equation.

$$
\text { (Eqn.3.1) } \quad \% C R=\left[\frac{P B_{0}-P B_{1}}{P B_{0}}\right] * 100
$$

$\mathrm{PB}$ is defined as Ablue/(Ablue $\left.+\mathrm{A}_{\mathrm{red}}\right){ }^{57,58} \mathrm{~PB}$ o was determined from the negative control of exposure of the vesicles to neat buffer, and $\mathrm{PB}_{1}$ was obtained is obtained from each experimental condition. A positive control of exposure to $100 \mu \mathrm{M} \mathrm{NaOH}$ was performed and used to standardize the PDA assays across independent trials. ${ }^{59} \mathrm{All}$ conditions were run in triplicate with a minimum of three independent experiments performed.

3.2.7 Atomic Force Microscopy (AFM). For ex situ AFM experiments, htt-exon1(46Q) $(10 \mu \mathrm{M})$ was incubated in the presence and absence of macromolecular crowders at $37^{\circ} \mathrm{C}$ and $1400 \mathrm{rpm}$ in an orbital thermomixer. At designated time points, $2 \mu \mathrm{L}$ aliquots of each sample were deposited onto freshly cleaved mica (Ted Pella Inc., Redding, CA) for 1 minute, washed with $200 \mu \mathrm{L}$ of $18 \mathrm{M} \Omega$ ultrapure water, and dried under a gentle stream of filtered air. For the fibril deposition experiment, htt-exon1(46Q) $(20 \mu \mathrm{M})$ was incubated at $37^{\circ} \mathrm{C}$ and $1400 \mathrm{rpm}$ in an orbital thermomixer for $24 \mathrm{~h}$ to pre-form fibrils. An aliquot of this sample was diluted into neat buffer (control) or macromolecular crowders 
to achieve a final concentration of $10 \mu \mathrm{M}$ of htt and $100 \mathrm{mg} / \mathrm{mL}$ of each crowder. These samples were deposited on mica as described above. All samples were imaged using a Nanoscope V Multi-Mode scanning probe microscope (VEECO) equipped with a closed loop vertical engage $\mathrm{J}$-scanner and silicon-oxide cantilevers with a resonance frequency of $\sim 300 \mathrm{kHz}$ and a nominal spring constant of $40 \mathrm{~N} / \mathrm{m}$. For in situ AFM experiments, a tapping mode fluid cell equipped with an $\mathrm{O}$ ring and cantilever with a nominal spring constant of $0.1 \mathrm{~N} / \mathrm{m}$ were used. For experiments of peptide aggregation on mica, samples of peptide $(10 \mu \mathrm{M})$ in neat buffer or with macromolecular crowders $(100 \mathrm{mg} / \mathrm{mL})$ were mixed and injected directly into the fluid cell. Aggregation on mica was monitored by continuous imaging. For experiments on lipid membranes, prepared vesicles were injected into the fluid cell and allowed to fuse onto the surface to form a supported bilayer. Once a continuous $40 \mu \mathrm{m} \times 40 \mu \mathrm{m}$ patch of supported lipid bilayer formed, httexon1(46Q) that had been incubated on ice with factor Xa for $1 \mathrm{~h}$ was injected with and without macromolecular crowders into the fluid cell for a final concentration of $10 \mu \mathrm{M}$ of htt and $25 \mathrm{mg} / \mathrm{mL}$ of crowders. Aggregation on the bilayer was monitored by continuous imaging. For in situ AFM experiments on mica or supported bilayers, scan size was periodically increased to ensure that the imaging process was not influencing aggregation. All AFM images, from both ex situ and in situ experiments, were analyzed using MATLAB equipped with the image processing toolbox (MathWorks) as described. 60,61

\subsection{Results}

3.3.1 Crowding alters htt aggregation in bulk solution. To investigate the impact of macromolecular crowders on htt aggregation in bulk solution, a GST-htt-exon1 fusion 
protein with 46 repeat glutamines (htt-exon1(46Q)) was used as a model system.

Cleavage of the GST tag with Factor Xa initiates aggregation. Htt-exon1(46Q) (10 $\mu \mathrm{M})$ was incubated in the presence of Dextran (20,000 da), Ficoll (400 da), or PEG $(20,000$ da), and fibril formation was monitored with a ThT assay (Figure 1). A concentration gradient $(50,100$, and $200 \mathrm{mg} / \mathrm{mL})$ was investigated for each crowder. For most conditions, there were not statistically significant changes in fibril formation compared to htt-exon1(46Q) incubated in the absence of crowders; although, there were some trends associated with each crowder. With dextran, ThT signal was typically larger than control, but this was only significantly different with $200 \mathrm{mg} / \mathrm{mL}$ dextran when comparing the maximum ThT signal $(p<0.05)$. Ficoll also appeared to increase fibril formation with increasing crowder concentration; however, the $200 \mathrm{mg} / \mathrm{mL}$ ficoll condition did not quite reach statistical significance. Unlike in the presence of the other two crowders, htt aggregation appeared slightly reduced in the presence of PEG, but this reduction did not reach statistical significance under any condition.
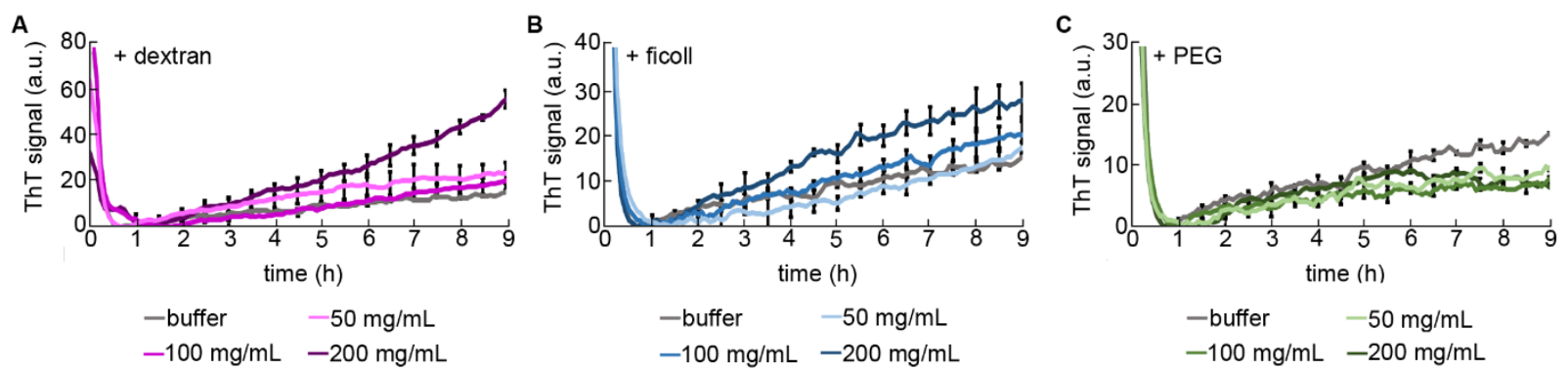

Figure 3.1: The impact of macromolecular crowders on htt-exon1(46Q) fibril formation as assessed by a ThT assay. Macromolecular crowders used were (A) dextran, (B) ficoll, and (C) $P E G$, and each was investigated at $50 \mathrm{mg} / \mathrm{mL}, 100 \mathrm{mg} / \mathrm{mL}$, and $200 \mathrm{mg} / \mathrm{mL}$. Htt-exon1(46Q) concentration was $20 \mu \mathrm{M}$. Buffer control represents experiments without any crowding agent. Error bars represent standard error of the mean (SEM) and are provided every $30 \mathrm{~min}$.

While the three crowders did not have a pronounced impact of fibril formation as measured by a ThT assay, this assay does not provide information concerning fibril 
morphology or other non-fibrillar aggregates such as oligomers. To determine if crowding impacted fibril morphology and oligomer formation, htt-exon1(46Q) incubations with macromolecular crowders and sampled after $8 \mathrm{~h}$ for AFM analysis. Httexon1(46Q) incubations in the absence of crowders were monitored as a control. Representative AFM images of these incubation with $100 \mathrm{mg} / \mathrm{mL}$ of each crowder are shown in Figure 2A, with background images of each crowder (no htt-exon1) deposited on mica are shown in Figure 3. Representative AFM images of htt-exon1(46Q) with other concentrations ( $50 \mathrm{mg} / \mathrm{mL}$ and $200 \mathrm{mg} / \mathrm{mL}$ ) of the crowders are presented in Figure 4. Based on the ThT assays, we expected to see significant populations of htt fibrils under all conditions; however, fibrils were only observed in the control incubation (Figure 2A). As a result, a control experiment was designed to determine if the crowders interfered with the deposition of fibrils onto mica (Figure 2B). $20 \mu \mathrm{M}$ htt-exon1(46Q) solutions were seeded with pre-formed fibrils and incubated to obtain extensive fibril populations. These pre-aggregated htt-exon1(46Q) samples were diluted to obtain a final protein concentration of $10 \mu \mathrm{M}$ with either neat buffer containing no crowding agents or with macromolecular crowders at a final concentration of $100 \mathrm{mg} / \mathrm{mL}$. After 5 min of mixing, these solutions were sampled and deposited on mica for AFM analysis of fibril coverage. Comparison of the number of fibrils per unit area for each sample demonstrated that all three crowders prevent fibrils from depositing on mica to a significant extent (Figure 2C). PEG had the largest impact on the fibril density on mica, reducing it by $92 \%$, followed by ficoll ( $73 \%$ reduction) and dextran ( $49 \%$ reduction). This analysis suggests that the absence of fibrils observed from incubations of httexon1(46Q) with each crowder may be due to interference with the deposition process. 
Despite the crowders interfering with fibril deposition, the different macromolecular crowders promoted distinct morphologies of non-fibrillar aggregates that adhered to mica (Figure 2A). To quantify this altered morphology, the height and diameter of each non-fibrillar aggregate was measured using automated scripts in MATLAB ${ }^{60,61}$ and used to construct height vs diameter correlation plots (Figure 2D). To verify that features observed in the AFM images derived from htt-exon1(46Q) were unique to the addition of protein, control crowder background images (Figure 4) were analyzed and also plotted in the correlation plots for comparison. Htt-exon1(46Q) in neat buffer formed a heterogeneous mixture of globular oligomers with heights ranging predominately from 1-8 $\mathrm{nm}$ and diameters ranging from $\sim 30-80 \mathrm{~nm}$. While the dextran background contained numerous features (heights $\sim 1-5 \mathrm{~nm}$, diameters $\sim 30-60 \mathrm{~nm}$ ) that overlapped with the size of the smaller htt-exon1 oligomers, a unique set of significantly larger globules were observed in incubations of htt-exon1(46Q) incubated with dextran at $100 \mathrm{mg} / \mathrm{mL}$. These large globules displayed tighter correlation between their heights and diameters with a significant population growing beyond the range (height $>10 \mathrm{~nm}$, diameters $>80 \mathrm{~nm}$ ) of features observed in the htt-exon1 control. These larger aggregates associated with htt-exon1 aggregation in the presence of dextran were present in the $50 \mathrm{mg} / \mathrm{mL}$ sample but were not observed at dextran concentration of 200 $\mathrm{mg} / \mathrm{mL}$.

Analysis of htt oligomers formed in the presence of ficoll is complicated due to the extensive features appearing in the ficoll blank control that had significant overlap with features observed when htt-exon1(46Q) was incubated with ficoll at $100 \mathrm{mg} / \mathrm{mL}$. However, there was a clearer shift of features distinct from the background at $50 \mathrm{mg} / \mathrm{mL}$ 
and $200 \mathrm{mg} / \mathrm{mL}$ ficoll experiments. Nevertheless, the distribution of potential httexon1(46Q) oligomers in the correlation plots associated with the presence of ficoll are distinct to those observed in the htt-exon1(46Q) control. With htt-exon1(46Q), very few features appeared on the mica surface in the presence of PEG, and few features appeared as background from control PEG experiments. However, observed oligomers of htt-exon1(46Q) formed in the presence of PEG at $100 \mathrm{mg} / \mathrm{mL}$ displayed a "fried egg" like morphology. That is, a flat feature around the periphery of each aggregated surrounded a distinct globular structure in the middle. While the measured height of these features were similar to oligomers observed in control htt-exon1(46Q) incubations, their diameters were significantly larger $(60-120 \mathrm{~nm})$ due to the peripheral portion of the aggregate. While ThT did not detect any difference associated with fibril formation and elongation, AFM images suggest that each crowder altered the morphology of aggregate intermediates to varying extent. This observation is limited, however, to the htt-exon1(46Q) aggregates that readily deposited on mica. 
A

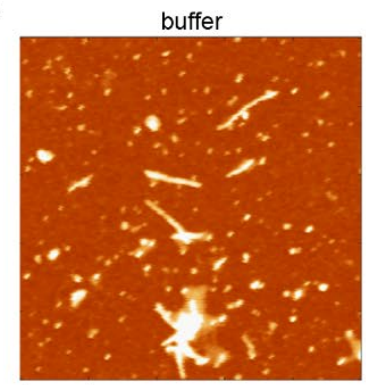
$500 \mathrm{~nm}$

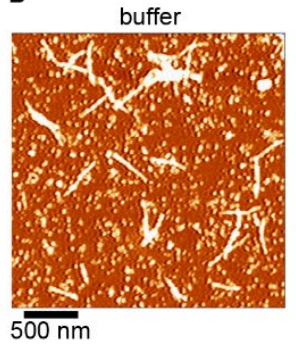

$+100 \mathrm{mg} / \mathrm{mL}$ dextran

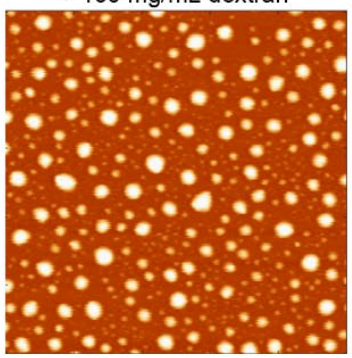

$+100 \mathrm{mg} / \mathrm{mL}$ dextran

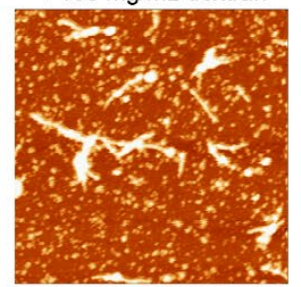

$+100 \mathrm{mg} / \mathrm{ML}$ ficoll

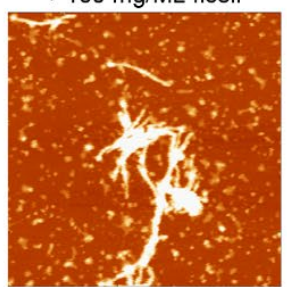

+ 100 mg/ML ficoll

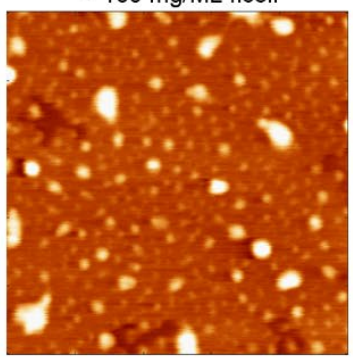

$+100 \mathrm{mg} / \mathrm{mL}$ PEG

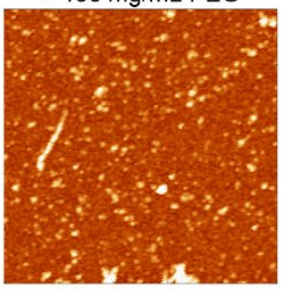

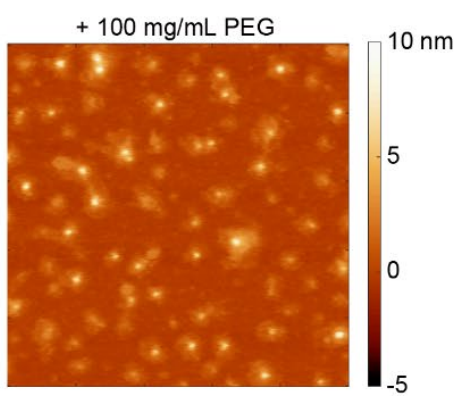

C

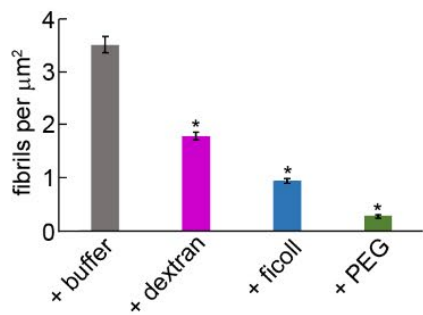

D *htt-exon1(46Q)
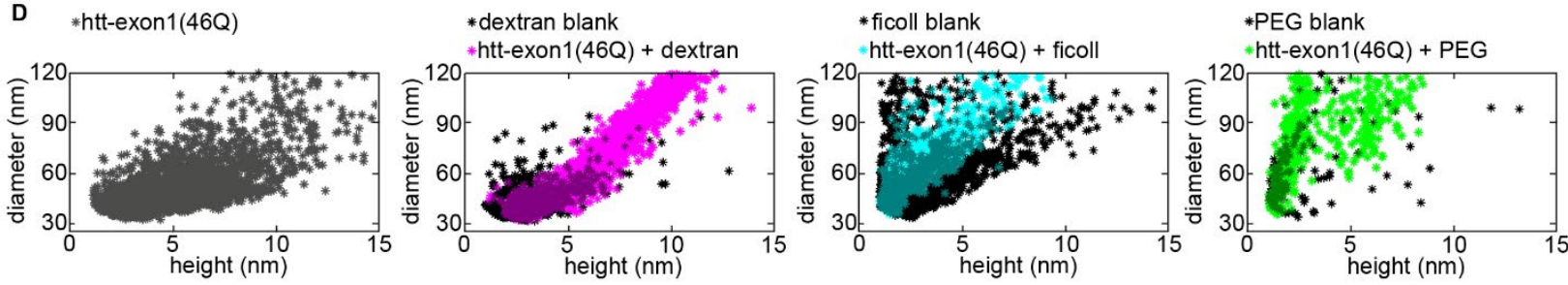

Figure 3.2: Ex situ AFM analysis of htt-exon1(46Q) aggregates formed in the presence of $100 \mathrm{mg} / \mathrm{ML}$ macromolecular crowders. Htt-exon1(46Q) was incubated at $20 \mu \mathrm{M}$ with dextran, ficoll, or PEG. These incubations were sampled for AFM imaging after $8 \mathrm{~h}$ of incubation, and (A) representative AFM images are presented. (B) Preformed htt-exon1(46Q) fibrils were mixed with $100 \mathrm{mg} / \mathrm{mL}$ macromolecular crowders, deposited on mica, and imaged by AFM to determine if the crowders interfered with the deposition of fibrils. (C) Quantification of the fibrils observed per unit area on mica from the fibril deposition experiment in (B). Error bars represent standard error of the mean (SEM), and * indicated $p<0.01$. (D) Correlation plots of height vs diameter of non-fibrillar aggregates observed in (A). For each correlation plot, features observed in the corresponding blank (crowder only) control are provided for comparison.

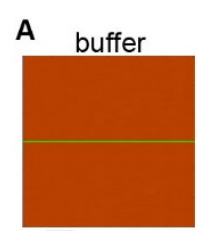

$5 \overline{00} \mathrm{~nm}$

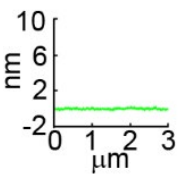

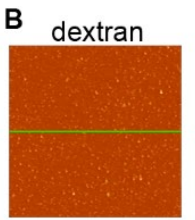

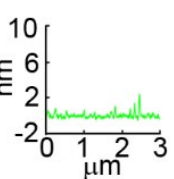

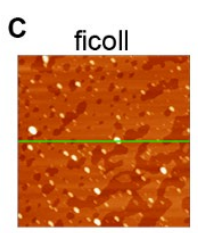

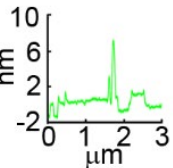

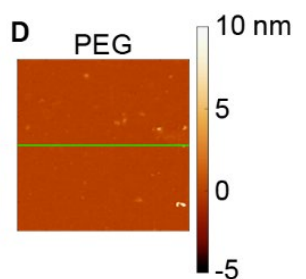

$\begin{array}{r}10 \\ 6 \\ 2 \\ 2 \\ -2 \\ \hline 0\end{array}$ 
Figure 3.3: Representative AFM images of mica exposed to $(A)$ neat buffer or $100 \mathrm{mg} / \mathrm{mL}$ of either (B) dextran, (C) ficoll, or (D) PEG. Height profiles, as indicated by the green lines, are provided below each image.
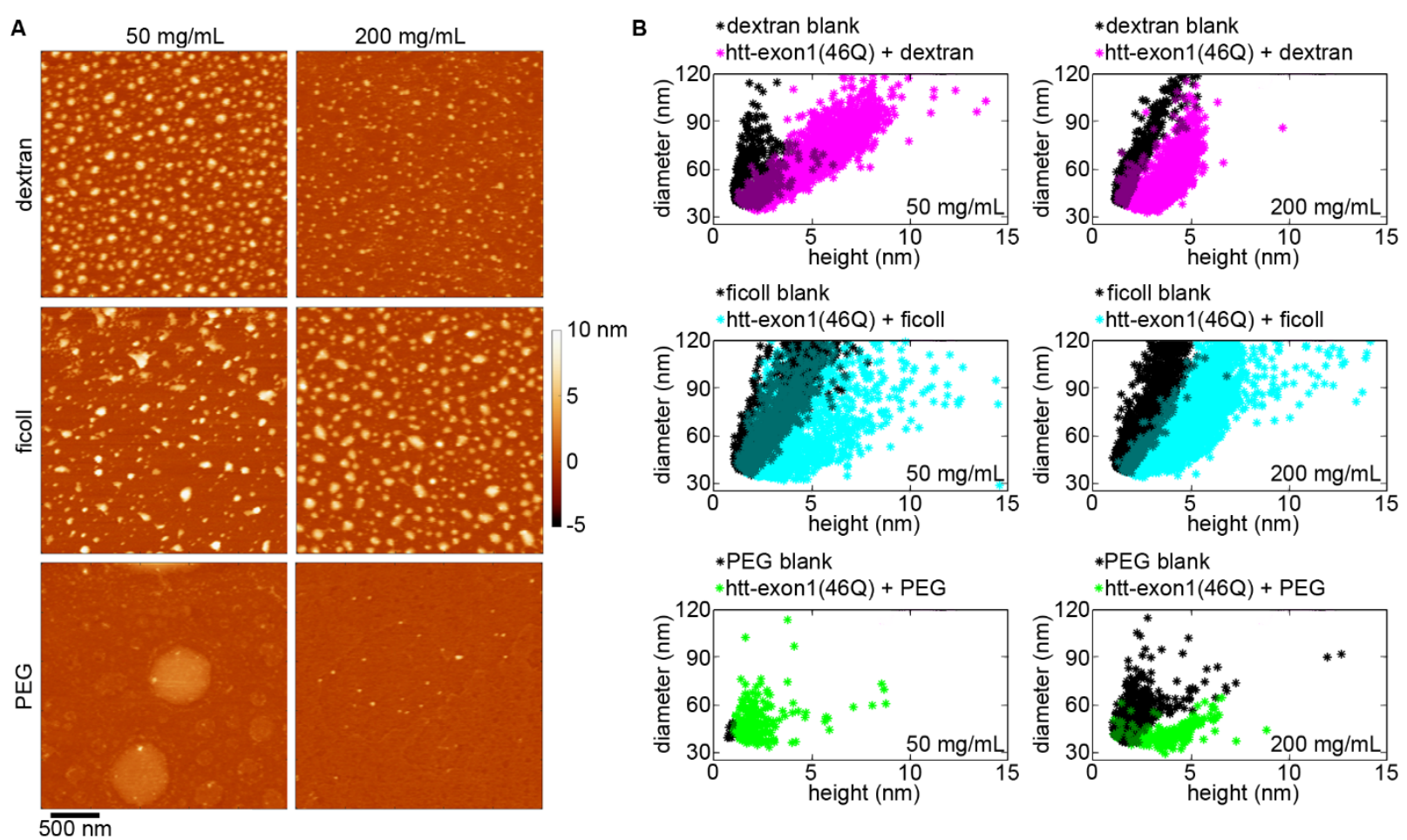

Figure 3.4: Ex situ AFM analysis of htt-exon1(46Q) aggregates formed in the presence of 50 or $100 \mathrm{mg} / \mathrm{ML}$ macromolecular crowders. Htt-exon1(46Q) was incubated at $20 \mu \mathrm{M}$ with dextran, ficoll, or PEG. These incubations were sampled for AFM imaging after $8 \mathrm{~h}$ of incubation, and $(A)$ representative AFM images are presented. (B) Correlation plots of height vs diameter of non-fibrillar aggregates observed in (A). For each correlation plot, features observed in the corresponding blank (crowder only) control are provided for comparison.

\subsubsection{Htt aggregation at a solid/liquid interface is modified by macromolecular}

crowders. As all three crowders interfered with fibril deposition onto mica and were associated with unique oligomeric species appearing on mica, it is possible that a crowded liquid phase alters the interaction of htt-exon1 with mica. As a result, we next aimed to elucidate if macromolecular crowding in the aqueous phase influenced aggregation occurring at a mica surface. Due to complications associated with the GST 
tag, a synthetic htt peptide that mimics htt-exon1 was used. This peptide was comprised of the first $\mathrm{N}$-terminal 17 amino acids (Nt17) of htt that precede the polyQ domain, a polyQ domain of 35 repeats, a polyP stretch of 10 residues, and two lysine residues at the C-terminus to aid in solubility. Due to this, the peptide is referred to as Nt17- $\mathrm{Q}_{35}-\mathrm{P}_{10-}$ KK. The real-time aggregation of $\mathrm{Nt} 17-\mathrm{Q}_{35}-\mathrm{P}_{10}-\mathrm{KK}$ on mica was tracked by continuous in situ AFM imaging (Figures 5 and 6). Solutions of Nt17-Q35-P10-KK were prepared in neat buffer or with crowders and injected directly into the AFM fluid cell to a final protein concentration of $10 \mu \mathrm{M}$ and crowder concentration of $100 \mathrm{mg} / \mathrm{mL}$.

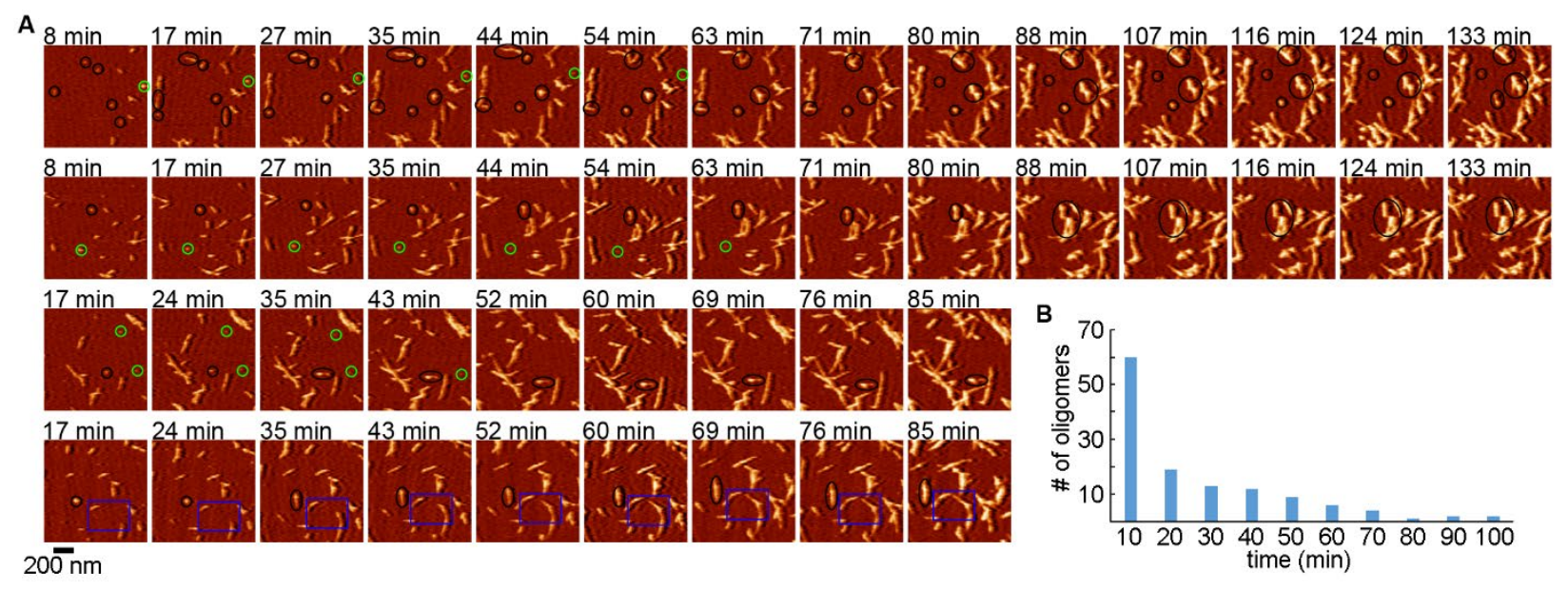

Figure 3.5: Tracking of individual Nt17- $Q_{35}-P_{10}-K K$ aggregates in mica as a function of time with no crowders in the aqueous phase. (A) A series of in situ AFM images of the same regions on mica. Black circles indicate aggregates in consecutive images that were initially classified as oligomers that remain stable or eventually transition into fibrils. Green circles identify oligomers in consecutive images that eventually desorb from the mica surface. The blue box indicates in consecutive images two fibrils that grow together. (B) Analysis of the time that 128 distinct oligomers remained stable on mica. 


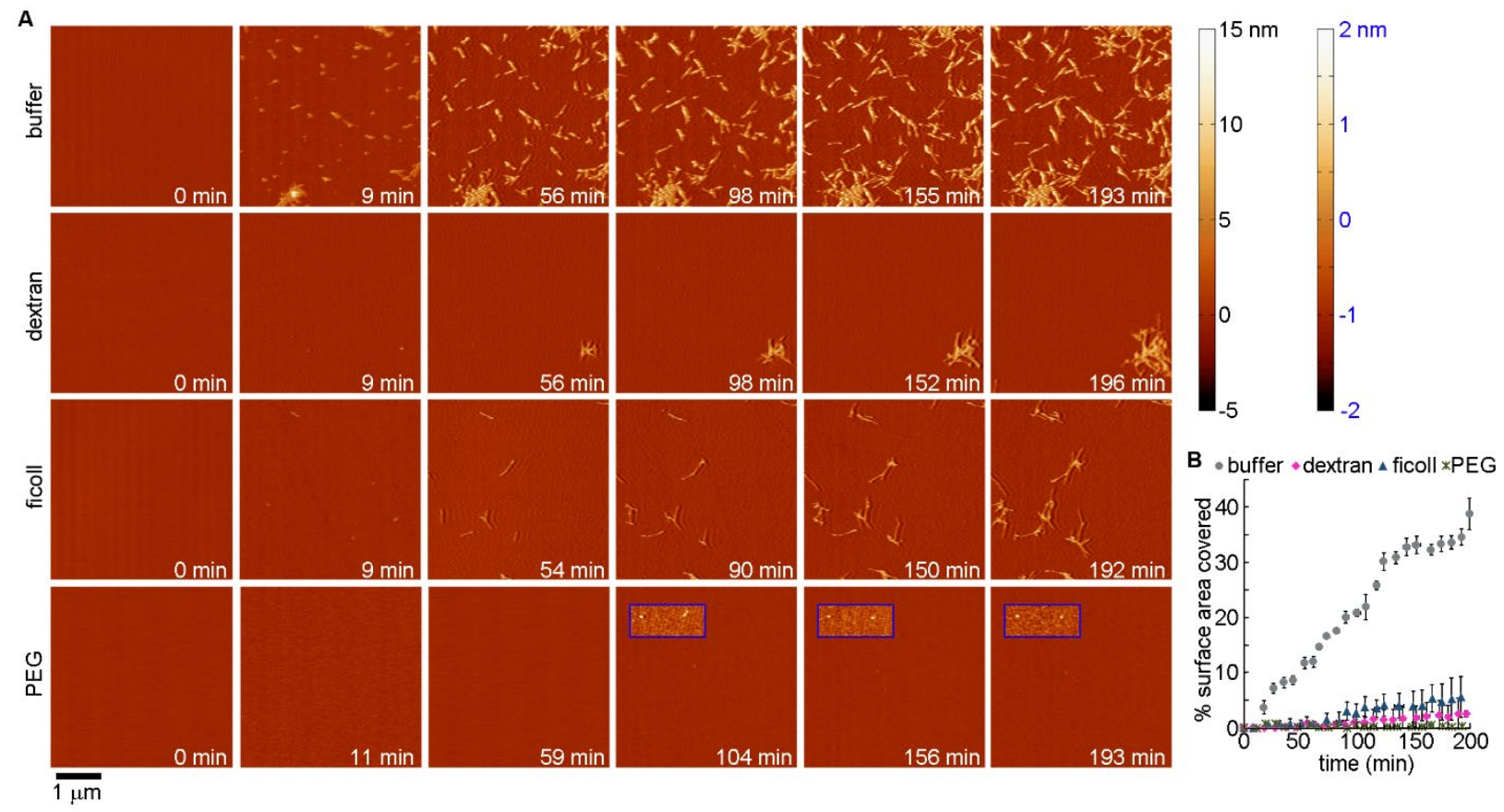

Figure 3.6: Comparison of Nt17- $Q_{35}-P_{10}-K K$ aggregation on mica with different macromolecular crowders in the aqueous phase. (A) Time-sequence, representative in situ AFM images of Nt17-Q ${ }_{35}-P_{10}-K K(10 \mu M)$ aggregating on mica with buffer, dextran, ficoll, or PEG in the aqueous phase. The macromolecular crowder concentration was $100 \mathrm{mg} / \mathrm{mL}$. As the height of features varied, the blue boxes represent areas of the image that correspond to the color scale with blue font. (B) Quantification of the \% surface area covered by Nt17-Q $Q_{35}-P_{10}-K K$ aggregates as a function of time. Error bars represent standard deviation.

Nt17-Q ${ }_{35}-P_{10}-K K$ in neat buffer (no crowders) formed a variety of oligomers and putative fibrils on mica within the timeframe of the first image ( $\sim-10$ minutes after injection, Figure 5 and $6 \mathrm{~A})$. The mica area occupied by these Nt17- $\mathrm{Q}_{35}-\mathrm{P}_{10}-\mathrm{KK}$ aggregates steadily increased with time, and the predominate aggregate species on the surface were fibrillar in morphology within 17-20 minutes. These fibrillar species grew in length from both ends as more peptide was incorporated into the aggregate. Branching was often observed, and fibrils growing into each other frequently occurred (Figure 5A). Several of these fibrils had clear oligomer precursors, but the majority of them did not. That is, they already had a fibrillar morphology upon their initial appearance. There were 
several observed mechanisms for oligomer disappearance. Some oligomers transitioned into fibrils, some desorbed from the mica surface, and others were incorporated into a pre-existing fibril that extended toward the oligomer. The relative stability of individual oligomers varied greatly. The fate of 128 individual oligomers were tracked from image to image to determine how long each remained in an oligomer conformation on the mica surface (Figure 5B). Just under half of these oligomers disappeared within $\sim 10$ minutes (or by the next image). However, $\sim 10 \%$ of identified oligomers persisted for over an hour on mica. The total amount of aggregation on mica was quantified by determining the percent surface area occupied by aggregates as a function of time (Figure 5B). Based on this analysis, total aggregation of Nt17-Q $35-\mathrm{P}_{10-}$ KK in neat buffer steadily grew, covering $39.2 \pm 2.9 \%$ of the mica surface after $\sim 200$ $\min$.

With the addition of macromolecular crowders in the aqueous phase, aggregation of Nt17-Q $35-\mathrm{P}_{10}-\mathrm{KK}$ on mica was significantly reduced (Figure $6 \mathrm{~A}$ ). With dextran and ficoll, a smaller number of aggregates (both oligomers and putative fibrils) appeared within 8-10 min compared to control. With time, fewer additional aggregates appeared on mica. The aggregates that did appear were mostly fibrillar and their morphology was indistinguishable to control. These fibrils elongated and branched with time. However, the extent of aggregation on the surface was significantly diminished, as only $2.6 \pm$ $0.5 \%$ and $5.6 \pm 3.7 \%$ of the mica surface was occupied by aggregates after $\sim 200 \mathrm{~min}$ (Figure 6B). With PEG, fibrils were not observed on mica, and only a few oligomers were present (occupying less than $0.5 \%$ of the total surface for over $200 \mathrm{~min}$; Figure $6 B)$. The observed oligomers in the presence of PEG were typically smaller than 
oligomers observed under all other conditions; however, very few of these small oligomers were observed across all trials with PEG, making comparisons difficult.

\subsubsection{Htt aggregation on lipid bilayers is modified by macromolecular crowders.}

As crowders altered htt peptide aggregation at a solid/liquid interface and lipid membranes can also impact amyloid formation, the ability of crowders in the aqueous phase to impact htt-exon1(46Q) on lipid bilayers was investigated. The model lipid system chosen for these studies was total brain lipid extract (TBLE) because it contains a physiologically relevant mixture of lipid components. To determine if the macromolecular crowders altered the ability of htt-exon1(46Q) to bind lipid membranes, TBLE/PDA vesicle lipid binding assay was performed (Figure 7). This is a colorimetric assay in which vesicles comprised of a mixture of phospholipids and polymerized PDA exhibit a rapid color change from blue to red due to mechanical stress induced by protein binding. By monitoring the \%CR peptide-membrane interactions and interfacial membrane processes can be quantified. First, each macromolecular crowder (at concentrations of 50,100 , and $200 \mathrm{mg} / \mathrm{mL}$ ) was tested to see if they invoked a CR in the TBLE/PDA vesicles in the absence of htt (Figure 8). The crowders did not invoke a CR in the absence of protein. Next, TBLE/PDA vesicles were exposed to httexon1(46Q) at $10 \mu \mathrm{M}$ in the presence of each crowder at the various concentrations, and the \%CR was measured over $9 \mathrm{~h}$ (Figure 7). Relative to control (htt-exon1(46Q) in the absence of crowders), all three crowders typically enhanced htt-exon1(46Q) interaction with TBLE vesicles. For dextran, the largest enhancement was observed at $50 \mathrm{mg} / \mathrm{mL}$ crowder concentration. At higher concentrations of dextran, htt-exon1(46Q) lipid binding was enhanced relative to control to a lesser extent. A similar trend was 
observed with Ficoll, as the lower concentrations of ficoll induced larger \%CRs.

Although, $200 \mathrm{mg} / \mathrm{mL}$ ficoll resulted in an initial jump in the \%CR that was followed by a slight reduced \%CR compared to control. PEG, at all concentrations, invoked an initial large response that dropped off after $\sim 1-2 \mathrm{~h}$. After this time frame, the \%CR either did not increase or tracked closely with control. A caveat is that the combination of PEG and htt-exon1(46Q) often caused the TBLE/PDA vesicles to fall out of solution, suggesting that the large initial response was destabilizing the suspension and causing the smaller measured \%CR. Nevertheless, the assay suggests that all three crowders enhanced the ability of htt-exon1(46Q) to bind vesicles.
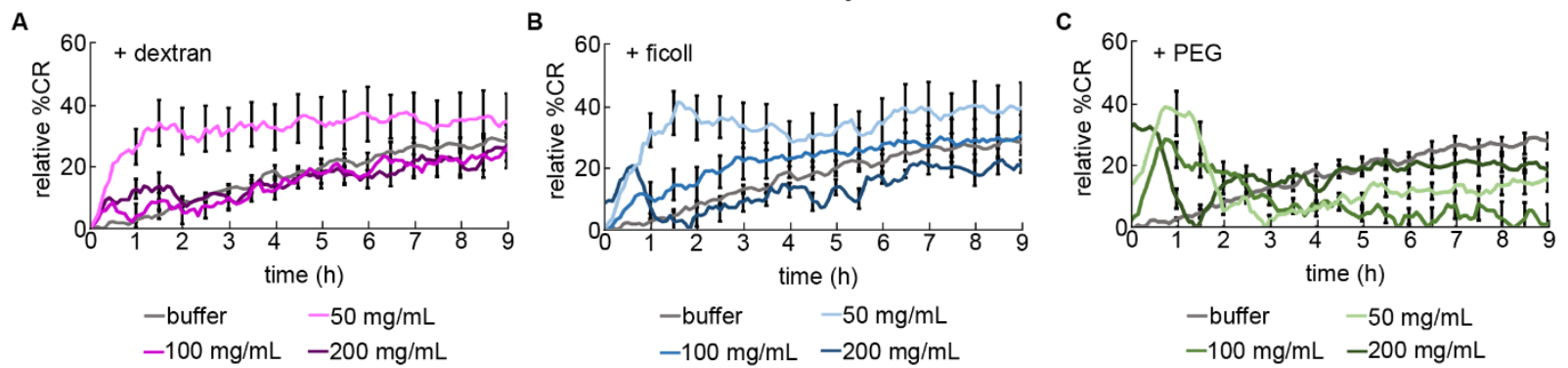

Figure 3.7. The impact of macromolecular crowders on htt-exon1(46Q) interaction with TBLE vesicles as assessed by a PDA assay. Macromolecular crowders used were (A) dextran, (B) ficoll, and (C) PEG, and each was investigated at $50 \mathrm{mg} / \mathrm{mL}, 100 \mathrm{mg} / \mathrm{mL}$, and $200 \mathrm{mg} / \mathrm{mL}$. Htt-exon1(46Q) concentration was $20 \mu \mathrm{M}$. Buffer control represents experiments without any crowding agent. Error bars represent standard error of the mean (SEM) and are provided every $30 \mathrm{~min}$. 

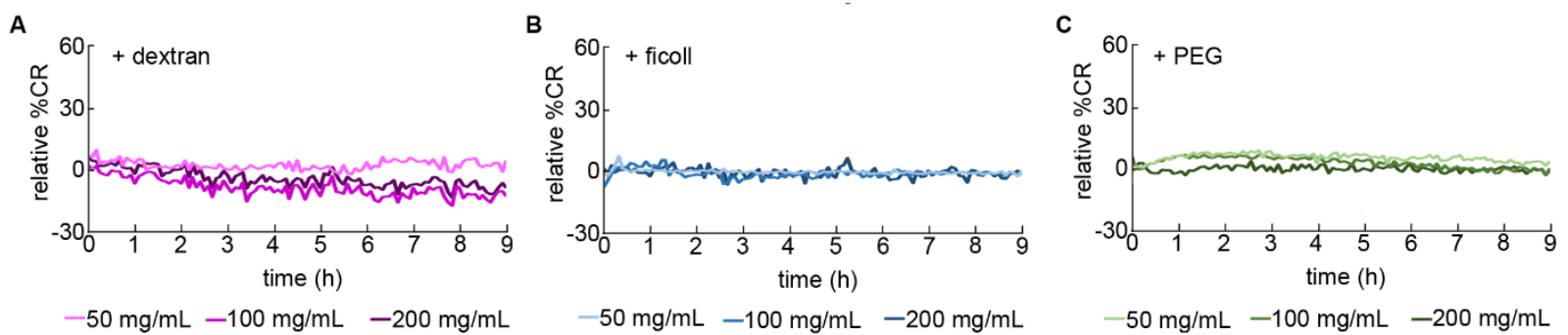

Figure 3.8. Control TBLE/PDA lipid binding assays assessing if macromolecular crowders invoke a colorimetric response. Macromolecular crowders used were $(A)$ dextran, (B) ficoll, and (C) PEG, and each was investigated at $50 \mathrm{mg} / \mathrm{mL}, 100 \mathrm{mg} / \mathrm{mL}$, and $200 \mathrm{mg} / \mathrm{mL}$.

As the macromolecular crowders altered the ability of htt-exon1(46Q) to interact with lipid vesicles, we next used a ThT assay to assess if these crowders altered fibril formation in the presence of TBLE vesicles (Figure 9). To further assess the impact of the crowders on fibrillization of htt, for these assays, the lipid to htt molar ratio was 10:1, and the vesicles were $\sim 140-180 \mathrm{~nm}$ in diameter as assessed by dynamic light scattering. The impact of the three crowders on fibril formation in the presence of TBLE vesicles as assessed by ThT followed a similar trend as that observed in the absence of TBLE vesicles. That is, dextran and ficoll appeared to increase fibrillization; PEG appeared to slightly inhibit fibrillization. However, with the addition of both vesicles and crowders, the noise in these assays was increased, and these differences did not reach statistical significance. However, the ThT assay comprehensively measures fibril formation of the entire sample and cannot distinguish between fibrils formed in bulk solution or at the vesicle surface. It also does not provide information regarding nonfibrillar aggregates. 

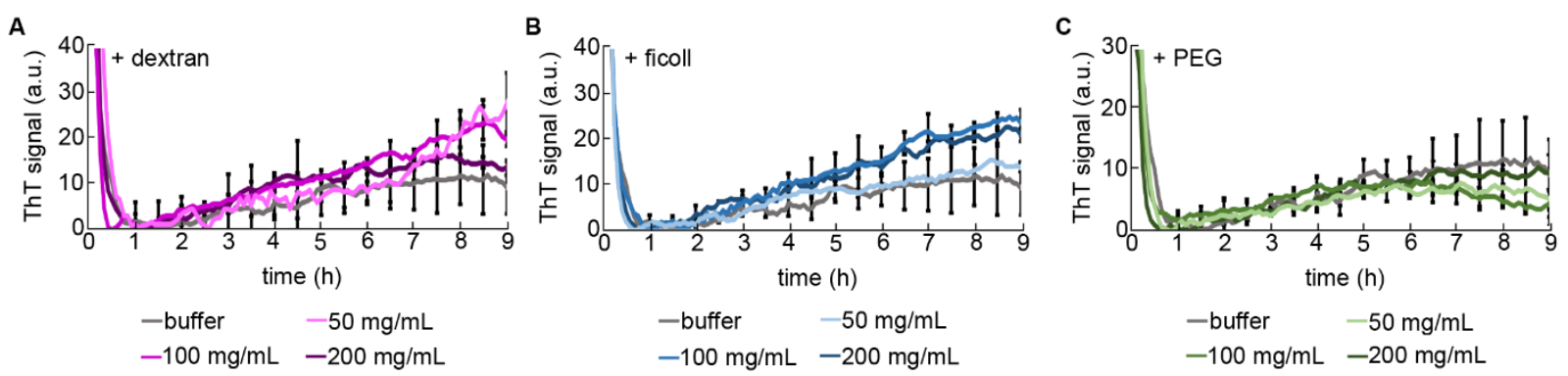

Figure 3.9. The impact of macromolecular crowders on htt-exon1(46Q) fibril formation in the presence of TBLE vesicles as assessed by a ThT assay. Macromolecular crowders used were (A) dextran, (B) ficoll, and (C) PEG, and each was investigated at $50 \mathrm{mg} / \mathrm{mL}, 100 \mathrm{mg} / \mathrm{mL}$, and $200 \mathrm{mg} / \mathrm{mL}$. Htt-exon1(46Q) concentration was $20 \mu \mathrm{M}$. TBLE vesicles were added at a lipid to peptide ratio of 10:1. Buffer control represents experiments without any crowding agent. Error bars represent standard error of the mean (SEM) and are provided every $30 \mathrm{~min}$.

To more directly investigate htt-exon1(46Q) aggregation on TBLE bilayers, in situ AFM was employed to observe aggregation occurring on a supported TBLE bilayer with a crowded aqueous phase (Figure 10). For these experiments, it is important that the supported bilayer is stable under continuous imaging. Therefore, control experiments were performed to verify that bilayers were stable when crowders were injected into the fluid cell. Supported bilayers were first formed on mica in neat buffer, and then macromolecular crowders were injected into the fluid cell. Based on these control experiments, it was determined that the supported bilayers were reliably imaged with up to $25 \mathrm{mg} / \mathrm{mL}$ of crowder in the fluid cell for several hours. Larger concentrations of crowders often resulted in membrane damage. As a result, these experiments were performed with a $25 \mathrm{mg} / \mathrm{mL}$ final concentration of crowding macromolecules. Only continuous TBLE bilayers ( $40 \times 40 \mu \mathrm{m}$ in size), as assessed by AFM imaging in neat buffer, were exposed to htt-exon1(46Q), and observations were limited to these verified continuous bilayers. A freshly prepared TBLE bilayer had an RMS roughness of $0.26 \pm 0.06 \mathrm{~nm}$ prior to being exposed to htt-exon1(46Q) (Figure 10B). Htt-exon1(46Q) 
was injected to a final concentration of $10 \mu \mathrm{M}$ in the fluid cell. For experiments with crowders, htt-exon1(46Q) and the crowder were mixed together and injected into the fluid cell at the same time.
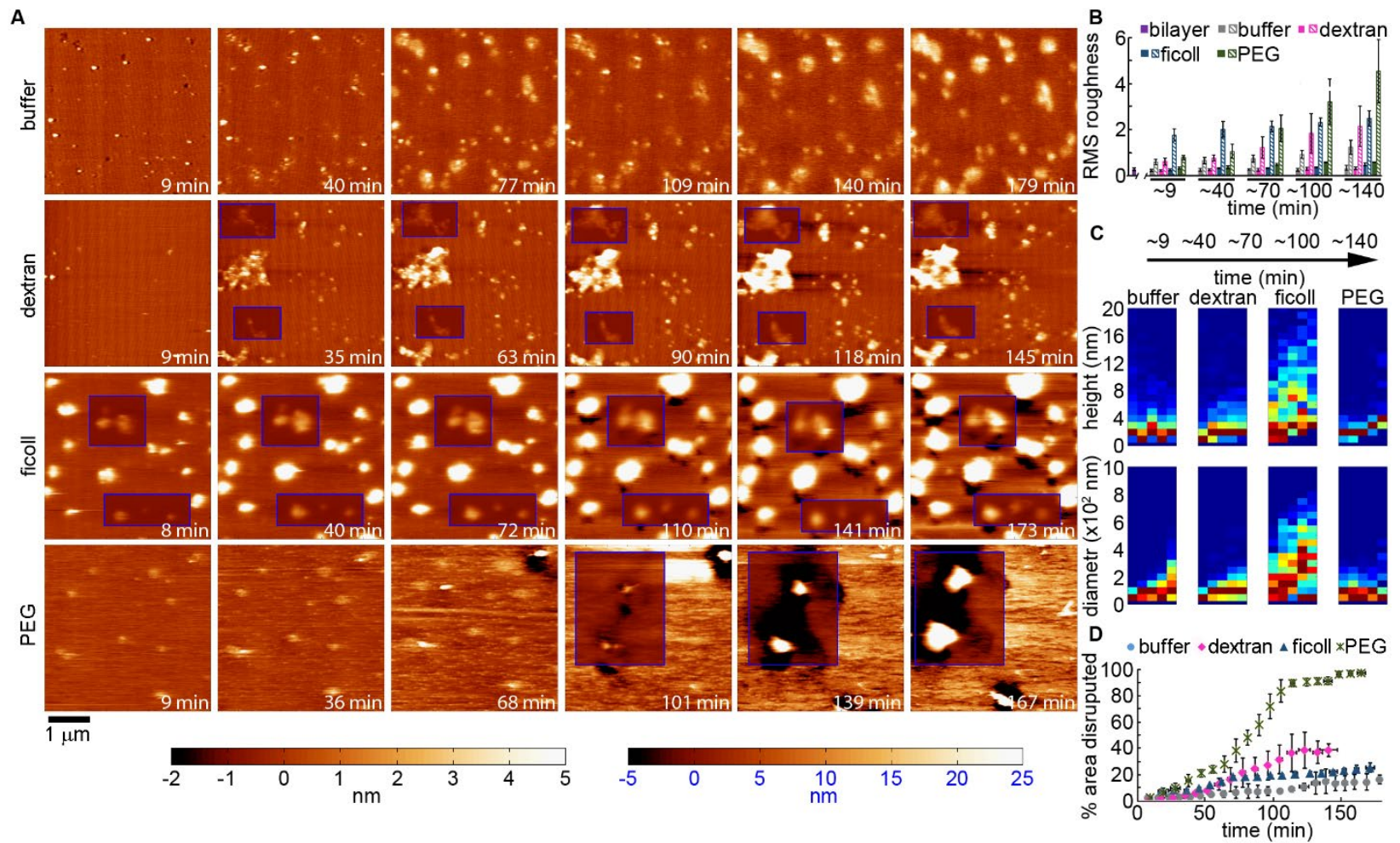

Figure 3.10. Comparison of htt-exon1(46Q) aggregation on supported TBLE bilayers with different macromolecular crowders in the aqueous phase. (A) Time-sequence, representative in situ AFM images of htt-exon1(46Q) (10 $\mu M$ ) aggregating on supported TBLE bilayers with buffer, dextran, ficoll, or PEG in the aqueous phase. The macromolecular crowder concentration was $25 \mathrm{mg} / \mathrm{mL}$. As the height of features varied, the blue boxes represent areas of the image that correspond to the color scale with blue font. (B) Quantification of the RMS roughness of unperturbed (solid bars) and disrupted (striped bars) regions of the bilayer. The RMS roughness of a bilayer prior to exposure to htt-exon1(46Q) is provided by the purple bar. Error bars represent standard deviation. (C) Height and diameter histograms of htt-exon1(46Q) aggregates as a function of time. (D) Quantification of the \% area of the bilayer disrupted by httexon1(46Q) as a function of time. Error bars represent standard deviation.

When TBLE was exposed to htt-exon1(46Q) in the absence of crowders, numerous oligomers appeared on the surface within $10 \mathrm{~min}$, and often small defects in the bilayer were observed (Figure 10A). With time, regions of the bilayer displayed 
disrupted morphology characterized by increased surface roughness and a highly granular appearance. Most of the bilayer maintained a smoother appearance. Often, these roughened areas developed around previously observed oligomers. These disrupted regions grew in area and became rougher with time. To quantify this roughening effect, regions of the bilayer displaying increased roughness were identified and the RMS roughness of these regions was determined (Figure 10B). The RMS roughness of the unaltered regions of the bilayer was also determined. The roughness of the smooth regions was comparable to freshly prepared TBLE bilayers. However, the RMS roughness of the disrupted portion of the membrane was $0.57 \pm 0.13 \mathrm{~nm}$ after $\sim 9$ $\mathrm{min}$, and this increased to $1.22 \pm 0.33 \mathrm{~nm}$ after $\sim 140 \mathrm{~min}$. The dimensions of the oligomers observed were also measured, and these oligomers swelled in size (predominately in the lateral dimension) with time (Figure 10C). To quantify the extent of the interaction of htt-exon1(46Q) with the supported bilayers, the percentage of the surface that displayed any altered morphology was determined as a function of time via AFM image analysis (Figure 10D). For this analysis, the area occupied with aggregates was considered as a disrupted region. After $\sim 130-145 \mathrm{~min}$, the extent of the bilayer disrupted leveled off at about $13-15 \%$ of the total bilayer area.

The interaction and aggregation of htt-exon1(46Q) with the supported TBLE bilayers was altered by the addition of macromolecular crowders in a crowder dependent manner. When the aqueous phase was crowded with dextran, oligomers appeared on the surface within the first 10 min of exposure to htt-exon1(46Q) in a similar fashion to the control experiment without crowders, and these oligomers increased in size with time (Figure 10A). Similar to control, regions of increased 
roughness with a granular appearance also developed on the bilayer, except these regions occupied more of the surface area with time (Figure 10C), and the RMS roughness of these regions became larger after $\sim 100$ min (Figure 10B). With Ficoll, httexon1(46Q) aggregation was quite different (Figure 10A). Large globular features appeared on the bilayer surface within the first 10 minutes. Rather than being associated with regions of a disrupted granular morphology, these large aggregates swelled in size, reaching heights as large as $20 \mathrm{~nm}$ above the bilayer surface and diameters on the order of 300-500 nm (Figure 10C). As these oligomers swelled, they occupied an increasing area of the bilayer. Additionally, holes spanning the entire bilayer appeared near these features within $\sim 100 \mathrm{~min}$, and these holes grew in size with time.

With PEG, regions of altered morphology formed on the bilayer within the first 10 min, but unlike the control, these regions were not granular in appearance (Figure 10A). Rather, they displayed a smoother, raised morphology. The bilayer between these regions was also slightly rougher $(0.33 \pm 0.04 \mathrm{~nm})$ than a typical TBLE bilayer (Figure 10B). By $\sim 30$ min with PEG, a considerably large population of small oligomers appear on the bilayer, and the regions of altered morphology grow. By $\sim 60 \mathrm{~min}$, large aggregates start to appear on the surface. Holes exposing the mica substrate developed around these large aggregates as they grew in size. With time, these holes covered a larger area (microns in size). Meanwhile, the rest of the bilayer became rougher, with greater than $95 \%$ of the surface displaying some type of altered morphology within $\sim 115$ min (Figure 10D).

\subsection{Discussion}


While toxicity has been attributed to numerous aggregate forms of htt, survival analysis of neurons expressing htt-exon 1 correlated diffusely distributed htt within the cell to cell death. However, this diffuse htt can be comprised of various types of aggregate species. For example, a significant population of htt oligomers are detectable in mouse and cellular models of HD that occupy this diffuse fraction. ${ }^{62,63}$ However, htt fibrils are also present in the diffuse fraction, as detected by super-resolution microscopy. ${ }^{64}$ Here, using simple macromolecules, we demonstrated that a crowded environment modifies the heterogeneous distribution of htt-exon1 aggregates formed in vitro. While the crowded environment within the cytosol is much more complicated, these results suggest that crowding effects likely exert a strong influence on the composition of the diffuse htt fraction linked to toxicity. In addition, potential toxic mechanisms attributed to htt aggregation involves damaging membranes associated with a variety of organelles, including the ER, mitochondria, and nuclear envelope. ${ }^{10,35,40,65}$ Here, we demonstrate that crowding in the aqueous phase alters the interaction with htt at solid/liquid and solid/membrane interfaces. In particular, the interactions with lipid membranes was enhanced in by crowding in the aqueous phase, and this was highly dependent on the chosen crowder. This suggests that the crowded environment of the cytosol can play an important role in htt/membrane interactions that are associated with toxicity.

There are several caveats in comparing the experiments on bilayers and mica. First, they used different models for htt aggregation, a synthetic $\mathrm{N}$-terminal fragment of htt shorter than exon1 on mica and a full htt-exon1 protein on bilayers. Second, the crowder concentrations that were feasible to use varied, $100 \mathrm{mg} / \mathrm{mL}$ with mica and 25 
$\mathrm{mg} / \mathrm{mL}$ with bilayers. However, there are some interesting difference worth noting. On mica, the extent of aggregates observed was altered, but with dextran and ficoll, the morphology of aggregates, especially fibrils, appearing on mica was identical to those observed in control. With PEG, only small oligomers were observed and not in great enough quantity to make meaningful comparisons. However, on TBLE bilayers, the different crowders promoted unique aggregate morphologies. The morphology of aggregates of polyQ containing peptides formed in the presence of mica is predominately influenced by polyQ length, not flanking sequences. ${ }^{3,30}$ On the other hand, a variety of membrane properties, lipid composition, and flanking sequences within htt-exon1 influence the interaction of htt with bilayers. ${ }^{30,47,51,66}$ This dichotomy of aggregation behavior between the two surfaces with different macromolecular crowders suggests more than a simple mechanism associated with protein diffusion via crowded environments. Indeed, the lack of meaningful crowder concentration dependent difference in fibril elongation as measured by ThT assays (both with and without lipids) supports that htt diffusion is likely not significantly inhibited, as impeded diffusion is known to slow amyloid elongation. ${ }^{67,68}$

All of the macromolecular crowders accelerated accumulation of htt-exon1(46Q) on TBLE bilayers within the first 1-2 $\mathrm{h}$ of exposure as measured the PDA assay and in situ AFM. This may be due to exclusion of htt-exon1(46Q) (and possibly membrane active aggregate species) from the solvent to the membrane interface. Localization and condensation of soluble proteins at lipid membrane interface is known to be sensitive to macromolecular crowding due to excluded volume effects. ${ }^{69,70}$ This excluded volume mechanism likely plays a larger role with dextran, as the htt-exon1(46Q) aggregates 
and bilayer morphological changes resembled that observed in the absence of crowders. Beyond a simple excluded volume explanation, there appeared to be crowder specific interactions associated with ficoll and PEG with htt-exon1(46Q) as each promoted unique aggregate morphologies and changes in the bilayer. This may be due to local concentration effects that promote protein/protein and/or protein/lipid interactions at the membrane/liquid interface. Their enhanced local concentration promotes protein:lipid and protein:protein interactions, oligomerization and/or aggregation, while soluble proteins and crowders that do not associate with the membrane are largely excluded from the interface ${ }^{71} \mathrm{In}$ this regard, changing macromolecular crowders enhance accumulation of RBC-modifying enzymes on cell surfaces up to 440 -fold. ${ }^{14}$

Considering the unique aggregate morphologies observed on TBLE with the addition of ficoll and PEG, a question arises as to if these aggregates formed in solution before binding the membrane or if they developed from smaller species once bound to the membrane. The imaging frequency of the continuous in situ AFM imaging was not fast enough to settle this question; however, it offers some hints toward answering this question. With ficoll, the large aggregate appeared within the timeframe to take the first image after injection of htt-exon1(46Q). These aggregates could have formed in solution, as the ex situ AFM analysis revealed similar sized features forming in the presence of ficoll, with large portions of these aggregates being unique from the ficoll background at 50 and $200 \mathrm{mg} / \mathrm{mL}$. This suggests that it is possible for these large features to have formed in solution prior to binding the surface. Once on the surface, these aggregates steadily grew in size and were associated with the development of 
holes in the bilayer, suggesting they may accumulate membrane lipids as well. While some morphological features on bilayers were associated with relatively large aggregates in the presence of PEG, most of the morphological changes were associated with smaller feature or ubiquitously spread across the bilayer, suggesting that a large portion of the htt-exon1(46Q) interacting with the bilayer was not extensively aggregated. All of these experiments were pre-formed with the same lipid system. The observed differences in aggregation as a function of crowder suggest that httexon1(46Q) localization to membrane interfaces is modified by the extent and type of crowding in the aqueous phase above the membrane surface.

\subsection{References}

(1) Macdonald, M. (1993) A novel gene containing a trinucleotide repeat that is expanded and unstable on Huntington's disease chromosomes. Cell 72, 971-983. (2) Wetzel, R. (2012) Physical chemistry of polyglutamine: Intriguing tales of a monotonous sequence. $J$ Mol Biol 421, 466-490.

(3) Legleiter, J., Mitchell, E., Lotz, G. P., Sapp, E., Ng, C., DiFiglia, M., Thompson, L. M., and Muchowski, P. J. (2010) Mutant Huntingtin Fragments Form Oligomers in a Polyglutamine Length-dependent Manner in Vitro and in Vivo. J Biol Chem 285, 1477714790.

(4) Adegbuyiro, A., Sedighi, F., Pilkington, A. W., Groover, S., and Legleiter, J. (2017) Proteins Containing Expanded Polyglutamine Tracts and Neurodegenerative Disease. Biochemistry 56, 1199-1217.

(5) Drombosky, K. W., Rode, S., Kodali, R., Jacob, T. C., Palladino, M. J., and Wetzel, R. (2018) Mutational analysis implicates the amyloid fibril as the toxic entity in Huntington's disease. Neurobiol Dis 120, 126-138.

(6) Pieri, L., Madiona, K., Bousset, L., and Melki, R. (2012) Fibrillar a-Synuclein and Huntingtin Exon 1 Assemblies Are Toxic to the Cells. Biophys J 102, 2894-2905.

(7) Lajoie, P., and Snapp, E. L. (2010) Formation and Toxicity of Soluble Polyglutamine Oligomers in Living Cells. PLoS ONE (Chirico, G., Ed.) 5, e15245.

(8) Nucifora, L. G., Burke, K. A., Feng, X., Arbez, N., Zhu, S., Miller, J., Yang, G., Ratovitski, T., Delannoy, M., Muchowski, P. J., Finkbeiner, S., Legleiter, J., Ross, C. A., and Poirier, M. A. (2012) Identification of Novel Potentially Toxic Oligomers Formed in Vitro from Mammalian-derived Expanded huntingtin Exon-1 Protein. J Biol Chem 287, 16017-16028.

(9) Kim, Y. E., Hosp, F., Frottin, F., Ge, H., Mann, M., Hayer-Hartl, M., and Hartl, F. U. (2016) Soluble Oligomers of PolyQ-Expanded Huntingtin Target a Multiplicity of Key Cellular Factors. Mol Cell 63, 951-964. 
(10) Bäuerlein, F. J. B., Saha, I., Mishra, A., Kalemanov, M., Martínez-Sánchez, A., Klein, R., Dudanova, I., Hipp, M. S., Hartl, F. U., Baumeister, W., and FernándezBusnadiego, R. (2017) In Situ Architecture and Cellular Interactions of PolyQ Inclusions. Cell 171, 179-187.e10.

(11) Guzman, I., and Gruebele, M. (2014) Protein Folding Dynamics in the Cell. J Phys Chem B 118, 8459-8470.

(12) Ellis, R. J., and Minton, A. P. (2003) Join the crowd. Nature 425, 27-28.

(13) Zimmerman, S. B., and Minton, A. P. (1993) Macromolecular Crowding:

Biochemical, Biophysical, and Physiological Consequences. Annu Rev Biophys Biomol Struct 22, 27-65.

(14) Chapanian, R., Kwan, D. H., Constantinescu, I., Shaikh, F. A., Rossi, N. A. . A., Withers, S. G., and Kizhakkedathu, J. N. (2014) Enhancement of biological reactions on cell surfaces via macromolecular crowding. Nat Commun 5, 4683.

(15) Friedman, R. (2011) Aggregation of amyloids in a cellular context: modelling and experiment. Biochem J 438, 415-426.

(16) Ellis, R. J. (2001) Macromolecular crowding: obvious but underappreciated. Trend Biochem Sci 26, 597-604.

(17) Sarkar, M., Li, C., and Pielak, G. J. (2013) Soft interactions and crowding. Biophys Rev 5, 187-194.

(18) Löwe, M., Kalacheva, M., Boersma, A. J., and Kedrov, A. (2020) The more the merrier: effects of macromolecular crowding on the structure and dynamics of biological membranes. FEBS $J$.

(19) Owen, M. C., Gnutt, D., Gao, M., Wärmländer, S. K. T. S., Jarvet, J., Gräslund, A., Winter, R., Ebbinghaus, S., and Strodel, B. (2019) Effects of in vivo conditions on amyloid aggregation. Chem Soc Rev 48, 3946-3996.

(20) Uversky, V. N., M Cooper, E., Bower, K. S., Li, J., and Fink, A. L. (2002) Accelerated alpha-synuclein fibrillation in crowded milieu. FEBS Lett 515, 99-103.

(21) Munishkina, L. A., Ahmad, A., Fink, A. L., and Uversky, V. N. (2008) Guiding protein aggregation with macromolecular crowding. Biochemistry 47, 8993-9006.

(22) Fung, J., Darabie, A. A., and McLaurin, J. (2005) Contribution of simple saccharides to the stabilization of amyloid structure. Biochem Biophys Res Commun 328, 1067-1072.

(23) Burke, K. A., Yates, E. A., and Legleiter, J. (2013) Biophysical Insights into How Surfaces, Including Lipid Membranes, Modulate Protein Aggregation Related to Neurodegeneration. Front Neurol 4.

(24) Moulin, A. M., O'Shea, S. J., Badley, R. A., Doyle, P., and Welland, M. E. (1999) Measuring Surface-Induced Conformational Changes in Proteins. Langmuir 15, 87768779.

(25) Gray, J. J. (2004) The interaction of proteins with solid surfaces. Curr Opin Struct Biol 14, 110-115.

(26) Goldsbury, C., Kistler, J., Aebi, U., Arvinte, T., and Cooper, G. J. S. (1999) Watching amyloid fibrils grow by time-lapse atomic force microscopy. $\mathrm{J} \mathrm{Mol} \mathrm{Biol} \mathrm{285,}$ 33-39.

(27) Hoyer, W., Cherny, D. I., Subramaniam, V., and Jovin, T. M. (2004) Rapid selfassembly of a-synuclein observed by in situ atomic force microscopy. $\mathrm{J} \mathrm{Mol} \mathrm{Biol} \mathrm{340,}$ 127-139. 
(28) Morriss-Andrews, A., and Shea, J.-E. (2012) Kinetic pathways to peptide aggregation on surfaces: The effects of $\beta$-sheet propensity and surface attraction. $J$ Chem Phys 136, 065103.

(29) Yates, E. A., Cucco, E. M., and Legleiter, J. (2011) Point Mutations in A $\beta$ Induce Polymorphic Aggregates at Liquid/Solid Interfaces. ACS Chem Neurosci 2, 294-307. (30) Burke, K. A., Kauffman, K. J., Umbaugh, C. S., Frey, S. L., and Legleiter, J. (2013) The Interaction of Polyglutamine Peptides with Lipid Membranes Is Regulated by Flanking Sequences Associated with Huntingtin. J Biol Chem 288, 14993-15005. (31) Gutekunst, C.-A., Li, S.-H., Yi, H., Mulroy, J. S., Kuemmerle, S., Jones, R., Rye, D., Ferrante, R. J., Hersch, S. M., and Li, X.-J. (1999) Nuclear and Neuropil Aggregates in Huntington's Disease: Relationship to Neuropathology. J Neurosci 19, 2522-2534. (32) Panov, A. V., Gutekunst, C.-A., Leavitt, B. R., Hayden, M. R., Burke, J. R., Strittmatter, W. J., and Greenamyre, J. T. (2002) Early mitochondrial calcium defects in Huntington's disease are a direct effect of polyglutamines. Nat Neurosci 5, 731-736. (33) Shirendeb, U., Reddy, A. P., Manczak, M., Calkins, M. J., Mao, P., Tagle, D. A., and Hemachandra Reddy, P. (2011) Abnormal mitochondrial dynamics, mitochondrial loss and mutant huntingtin oligomers in Huntington's disease: implications for selective neuronal damage. Hum Mol Genet 20, 1438-1455.

(34) Ueda, M., Li, S., Itoh, M., Wang, M., Hayakawa, M., Islam, S., Tana, Nakagawa, K., Chen, H., and Nakagawa, T. (2016) Expanded polyglutamine embedded in the endoplasmic reticulum causes membrane distortion and coincides with Bax insertion. Biochem Biophys Res Co 474, 259-263.

(35) Orr, A. L., Li, S., Wang, C.-E., Li, H., Wang, J., Rong, J., Xu, X., Mastroberardino, P. G., Greenamyre, J. T., and Li, X.-J. (2008) N-Terminal Mutant Huntingtin Associates with Mitochondria and Impairs Mitochondrial Trafficking. J Neurosci 28, 2783-2792. (36) Kegel-Gleason, K. B. (2013) Huntingtin Interactions with Membrane Phospholipids: Strategic Targets for Therapeutic Intervention? JHD 2, 239-250.

(37) Lee, W.-C. M., Yoshihara, M., and Littleton, J. T. (2004) Cytoplasmic aggregates trap polyglutamine-containing proteins and block axonal transport in a Drosophila model of Huntington's disease. PNAS 101, 3224-3229.

(38) Burke, K. A., Hensal, K. M., Umbaugh, C. S., Chaibva, M., and Legleiter, J. (2013) Huntingtin disrupts lipid bilayers in a polyQ-length dependent manner. BBA Biomembranes 1828, 1953-1961.

(39) Suopanki, J., Götz, C., Lutsch, G., Schiller, J., Harjes, P., Herrmann, A., and Wanker, E. E. (2006) Interaction of huntingtin fragments with brain membranes - clues to early dysfunction in Huntington's disease. J Neurochem 96, 870-884.

(40) Choo, Y. S., Johnson, G. V. W., MacDonald, M., Detloff, P. J., and Lesort, M. (2004) Mutant huntingtin directly increases susceptibility of mitochondria to the calciuminduced permeability transition and cytochrome c release. Hum Mol Genet 13, 14071420.

(41) Eckmann, J., Clemens, L. E., Eckert, S. H., Hagl, S., Yu-Taeger, L., Bordet, T., Pruss, R. M., Muller, W. E., Leuner, K., Nguyen, H. P., and Eckert, G. P. (2014) Mitochondrial membrane fluidity is consistently increased in different models of Huntington disease: restorative effects of olesoxime. Mol Neurobiol 50, 107-118. (42) Qin, Z.-H. (2004) Huntingtin Bodies Sequester Vesicle-Associated Proteins by a Polyproline-Dependent Interaction. J Neurosci 24, 269-281. 
(43) Kegel, K. B., Kim, M., Sapp, E., McIntyre, C., Castaño, J. G., Aronin, N., and DiFiglia, M. (2000) Huntingtin Expression Stimulates Endosomal-Lysosomal Activity, Endosome Tubulation, and Autophagy. J Neurosci 20, 7268-7278.

(44) Valencia, A., Reeves, P. B., Sapp, E., Li, X., Alexander, J., Kegel, K. B., Chase, K., Aronin, N., and DiFiglia, M. (2010) Mutant huntingtin and glycogen synthase kinase 3beta accumulate in neuronal lipid rafts of a presymptomatic knock-in mouse model of Huntington's disease. J Neurosci Res 88, 179-190.

(45) Tao, M., Pandey, N. K., Barnes, R., Han, S., and Langen, R. (2019) Structure of Membrane-Bound Huntingtin Exon 1 Reveals Membrane Interaction and Aggregation Mechanisms. Structure 27, 1570-1580.e4.

(46) Beasley, M., Stonebraker, A. R., Hasan, I., Kapp, K. L., Liang, B. J., Agarwal, G., Groover, S., Sedighi, F., and Legleiter, J. (2019) Lipid Membranes Influence the Ability of Small Molecules To Inhibit Huntingtin Fibrillization. Biochemistry 58, 4361-4373. (47) Gao, X., Campbell, W. A., Chaibva, M., Jain, P., Leslie, A. E., Frey, S. L., and Legleiter, J. (2016) Cholesterol Modifies Huntingtin Binding to, Disruption of, and Aggregation on Lipid Membranes. Biochemistry 55, 92-102.

(48) Chaibva, M., Gao, X., Jain, P., Campbell, W. A., Frey, S. L., and Legleiter, J. (2018) Sphingomyelin and GM1 Influence Huntingtin Binding to, Disruption of, and Aggregation on Lipid Membranes. ACS Omega 3, 273-285.

(49) Pandey, N. K., Isas, J. M., Rawat, A., Lee, R. V., Langen, J., Pandey, P., and Langen, $R$. (2018) The 17-residue-long $N$ terminus in huntingtin controls stepwise aggregation in solution and on membranes via different mechanisms. J Biol Chem 293, 2597-2605.

(50) Marquette, A., and Bechinger, B. (2020) Membrane interactions accelerate the selfaggregation of huntingtin exon 1 fragments in a polyglutamine length-dependent manner. Biophysics.

(51) Beasley, M., Groover, S., Valentine, S. J., and Legleiter, J. (in press) Lipid headgroups alter huntingtin aggregation on membranes. BBA-Biomembranes.

(52) Levy, G. R., Shen, K., Gavrilov, Y., Smith, P. E. S., Levy, Y., Chan, R., Frydman, J., and Frydman, L. (2019) Huntingtin's N-Terminus Rearrangements in the Presence of Membranes: A Joint Spectroscopic and Computational Perspective. ACS Chem Neurosci 10, 472-481.

(53) Ganji, M., Docter, M., Le Grice, S. F. J., and Abbondanzieri, E. A. (2016) DNA binding proteins explore multiple local configurations during docking via rapid rebinding. Nucleic Acids Res 44, 8376-8384.

(54) Tokuriki, N., Kinjo, M., Negi, S., Hoshino, M., Goto, Y., Urabe, I., and Yomo, T. (2004) Protein folding by the effects of macromolecular crowding. Protein Sci 13, 125133.

(55) Muchowski, P. J., Schaffar, G., Sittler, A., Wanker, E. E., Hayer-Hartl, M. K., and Hartl, F. U. (2000) Hsp70 and hsp40 chaperones can inhibit self-assembly of polyglutamine proteins into amyloid-like fibrils. Proc Natl Acad Sci USA 97, 7841-7846. (56) Chen, S. (2001) Solubilization and disaggregation of polyglutamine peptides. Protein Sci 10, 887-891.

(57) Zheng, F., Wu, Z., and Chen, Y. (2012) A quantitative method for the measurement of membrane affinity by polydiacetylene-based colorimetric assay. Anal Biochem 420, $171-176$. 
(58) Sokolovski, M., Sheynis, T., Kolusheva, S., and Jelinek, R. (2008) Membrane interactions and lipid binding of casein oligomers and early aggregates. BBA -

Biomembranes 1778, 2341-2349.

(59) Beasley, M., Stonebraker, A. R., and Legleiter, J. Normalizing Polydiacetylene Colorimetric Assays of Vesicle Binding Across Lipid Systems. Anal Biochem (in press).

(60) Burke, K. A., Godbey, J., and Legleiter, J. (2011) Assessing mutant huntingtin fragment and polyglutamine aggregation by atomic force microscopy. Methods 53, 275284.

(61) Burke, K. A., and Legleiter, J. (2013) Atomic force microscopy assays for evaluating polyglutamine aggregation in solution and on surfaces. Methods $\mathrm{Mol} \mathrm{Biol}$ 1017, 21-40.

(62) Ma, O., Lm, A., Ym, R., J, T., Mf, B., Af, H., and Dm, H. (2010) Tracking mutant huntingtin aggregation kinetics in cells reveals three major populations that include an invariant oligomer pool. J Biol Chem 285, 21807-21816.

(63) Marcellin, D., Abramowski, D., Young, D., Richter, J., Weiss, A., Marcel, A., Maassen, J., Kauffmann, M., Bibel, M., Shimshek, D. R., Faull, R. L. M., Bates, G. P., Kuhn, R. R., Putten, P. H. V. der, Schmid, P., and Lotz, G. P. (2012) Fragments of HdhQ150 Mutant Huntingtin Form a Soluble Oligomer Pool That Declines with Aggregate Deposition upon Aging. PLOS ONE 7, e44457.

(64) Sahl, S. J., Weiss, L. E., Duim, W. C., Frydman, J., and Moerner, W. E. (2012) Cellular Inclusion Bodies of Mutant Huntingtin Exon 1 Obscure Small Fibrillar Aggregate Species. Scientific Reports 2, 895.

(65) Liu, K.-Y., Shyu, Y.-C., Barbaro, B. A., Lin, Y.-T., Chern, Y., Thompson, L. M., James Shen, C.-K., and Marsh, J. L. (2015) Disruption of the nuclear membrane by perinuclear inclusions of mutant huntingtin causes cell-cycle re-entry and striatal cell death in mouse and cell models of Huntington's disease. Hum Mol Genet 24, 16021616.

(66) Chaibva, M., Burke, K. A., and Legleiter, J. (2014) Curvature Enhances Binding and Aggregation of Huntingtin at Lipid Membranes. Biochemistry 53, 2355-2365.

(67) Phillip, Y., and Schreiber, G. (2013) Formation of protein complexes in crowded environments - From in vitro to in vivo. FEBS Letters 587, 1046-1052.

(68) Phillip, Y., Kiss, V., and Schreiber, G. (2012) Protein-binding dynamics imaged in a living cell. PNAS 109, 1461-1466.

(69) Minton, A. P., Colclasure, G. C., and Parker, J. C. (1992) Model for the role of macromolecular crowding in regulation of cellular volume. Proc Natl Acad Sci USA 89, 10504-10506.

(70) Kim, J. S., and Yethiraj, A. (2010) Crowding Effects on Association Reactions at Membranes. Biophys J 98, 951-958.

(71) Nawrocki, G., Im, W., Sugita, Y., and Feig, M. (2019) Clustering and dynamics of crowded proteins near membranes and their influence on membrane bending. PNAS 116, 24562-24567. 


\section{Future Work and Concluding Remarks}

The work presented in this dissertation was aimed at elucidating interactions of htt with surfaces, based on an electrostatic and lipid interface perspectives. To begin, an experimental system was established using mutations in Nt17 to htt phosphorylation. Once this system was validated, investigations were aimed at understanding the impact of phosphorylation on $\mathrm{htt} /$ lipid interactions with a variety of lipid systems. Understanding biophysical impact of phosphomimetic mutations on htt aggregation and lipid membrane activity may provide mechanistic detail regarding htt membrane association linked to toxicity. This was followed by a study aimed at elucidating how a crowded liquid phase influences htt aggregation at interfaces. A variety of macromolecular crowders were used, and each had a profound impact on aggregation at a mica/liquid interfaces and a membrane/liquid interface. The major goal of these studies individually was to understand fundamental aspects of htt aggregation, including charge and membrane interactions, in the presence of cellular and subcellular surfaces. The ultimate overall goal was to identify and expand upon potential targets for preventing mutant huntingtin aggregation; hopefully leading to more available, effective therapeutic and preventative treatments for HD. To further the work completed in this dissertation in context, implications for future studies are discussed.

\subsection{Impacts of PIP Lipids and Htt on Membranes}

$\mathrm{Htt}$ is associated with a variety of distinct membranes within neurons, each comprised of a distinct mix of lipid components. While htt can damage these different membranes, different aggregate species have been associated with specific organelles. 
For example, oligomers have been implicated in mitochondrial membrane dysfunction; ${ }^{1,2}$ fibrils deform and damage the $\mathrm{ER} ;{ }^{3}$ and inclusion disrupt the nuclear envelope. ${ }^{4}$ Thus, it is important to understand how different lipid species influence $\mathrm{htt} / \mathrm{membrane}$ interactions and aggregation. Nt17 forms a membrane targeting $\mathrm{AH}$, which facilities htt-exon1's direct association with a variety of cellular and subcellular membranes via direct contact with phospholipids within the membrane. ${ }^{5-7}$ For example, mutant htt has increased binding affinity to numerous phospholipids, ${ }^{6,7}$ and mutant htt fragments can directly disrupt phospholipid bilayer integrity. ${ }^{7} \mathrm{Htt}$ has displayed a high affinity for a small class of lipids called phosphatidylinositol phosphates (PIPs). Of the seven known PIPs, the low abundance $\left(0.1 \%\right.$ of total lipids) multivalent PIPs, $\mathrm{PI}(3,4) \mathrm{P}_{2}$, $\mathrm{PI}(3,5) \mathrm{P}_{2}, \mathrm{PI}(4,5) \mathrm{P}_{2}$ and $\mathrm{PI}(3,4,5) \mathrm{P}_{2}$, associate more strongly with htt. ${ }^{7-9}$ Due to the positive charge associated with the lysine residues in Nt17 which are integral to lipid binding; ${ }^{10}$ and the negative charge of PIPs, it is possible that lysines within Nt17 underscore the high affinity of htt for PIP enriched membranes.

Charged phospholipids, such as PIPs, interact with ions via Coulombic forces. Divalent cations and $\mathrm{Li}^{+}$with POPC can change mechanics and shift membranes to a more gel-like state. ${ }^{11}$ It is then expected that cation induced clustering of PIP with $\mathrm{Ca}^{+2}$ should rigidify the membrane by diminishing lateral diffusion, reduce leakage, and lessen the damage when htt is bound to the membrane. It is feasible to expect that changing the ionic strength could have an effect on how the membrane interacts with the htt protein. $\mathrm{PIP}_{2}$ forms clusters in vitro. ${ }^{12,13}$ Most models with PIP clustering use higher concentrations of divalent ions and PIP lipids greater than what is naturally present in the plasma membrane. ${ }^{12,14,15}$ To date, the effects of PIP clustering on htt 
binding and aggregation are unknown. Calcium clearly plays a vital role in both PIP lipids and htt, but little evidence has been presented at physiological concentrations.

This clustering would have additional consequences in downstream applications of htt, for example, PIP enriched membranes play a key regulatory role in AKTmediated phosphorylation of htt. ${ }^{7-9}$ While the interaction of primary PIPs, such as $\mathrm{Pl}(4,5) \mathrm{P}_{2}$, with htt has been analyzed in the literature ${ }^{16}$, the structural changes resulting from interaction of secondary multivalent $\mathrm{PIPs} \mathrm{PI}(3,4) \mathrm{P}_{2}$ and $\mathrm{PI}(3,4,5) \mathrm{P}_{3}$ with htt has not been explored in depth. Most studies focus toward animal models and immune or cytotoxicity evidence that can be related back to the kinases and pathways, ${ }^{17,18}$ with little information on the actual binding event that initiates these processes. A model for htt recruitment to the plasma membrane by PIPs during growth factor signaling shows that the type III kinase uses $\mathrm{PI}(4) \mathrm{P}_{2}$ and $\mathrm{PI}(4,5) \mathrm{P}_{2}$ as substrates to create $\mathrm{PI}(3,4) \mathrm{P}_{2}$ and $\mathrm{PI}(3,4,5) \mathrm{P}_{3}$ at the membrane surface.${ }^{16}$ Experiments with lipid overlay indicate that $\mathrm{htt}$ binds multivalent PIPs which is consistent with an electrostatic mode of association. ${ }^{8}$ PIPs provide a high affinity binding site on the inner cytosolic membrane surface for proteins like AKT and htt. ${ }^{9} \mathrm{Htt}$ is a substrate for AKT and phosphorylation of htt by AKT is crucial for neuroprotective effects but is altered by mutant htt. ${ }^{19}$ Due to PIPs clustering ability with $\mathrm{Ca}^{+2}$, it appears that mutant $\mathrm{htt}$ and AKT could accumulate along with GSK $\beta$ to activate cell death pathways with mutant $\mathrm{htt}^{7}$ 


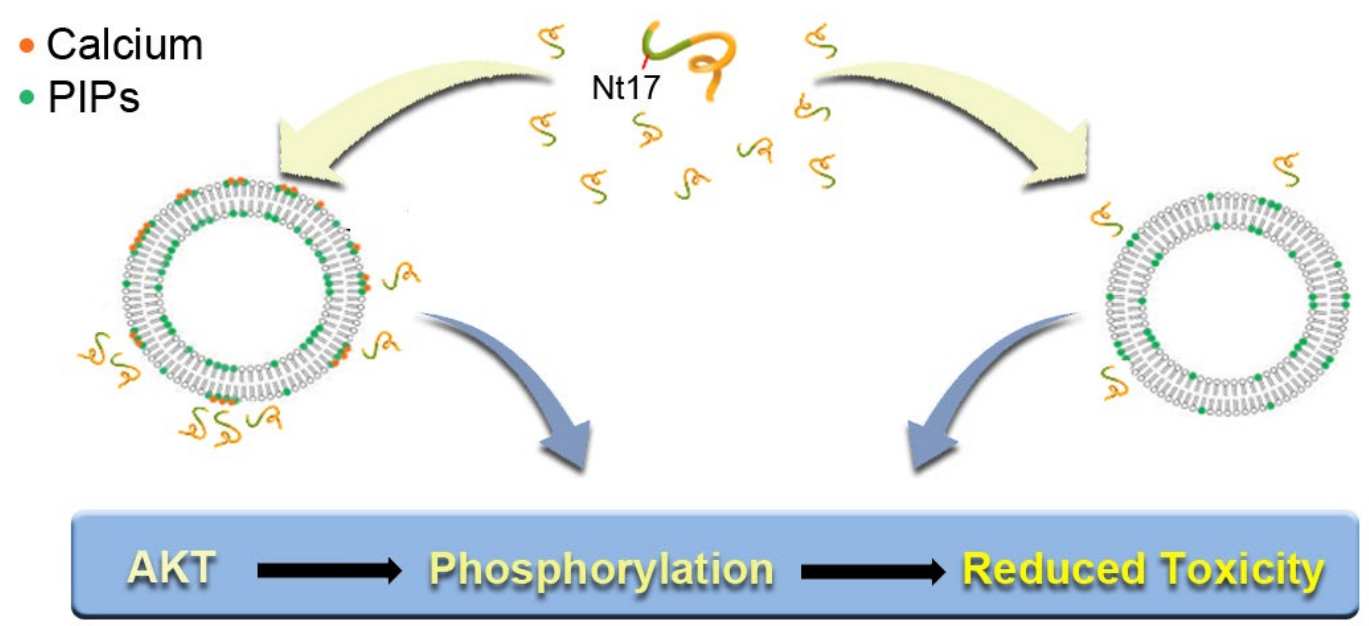

Figure 4.1: Proposed interaction of Nt17 association with PIP and PIP/Ca ${ }^{+2}$ vesicles. The binding of Nt17 to PIP/Ca ${ }^{+2}$ clusters on a lipid membrane (left) can expect to have a stronger response than the binding of Nt17 to PIP enhanced lipid membranes (right). This is due to increased electrostatic interactions, however both proposed interactions lead to downstream therapeutic pathways focused on reduced toxicity.

Administration of PIPs or altering PIP kinases as a therapeutic target in animal models have included direct application to the brain, altering embryonic or similar developing cells in animal models ${ }^{16}$ or non-physiological conditions, ${ }^{20}$ which is unrealistic for future treatment of HD. Recent efforts have shown that increasing the levels of PIP decreases the amount of protein aggregation associated with several neurodegenerative diseases, ${ }^{16}$ but little is known about the mechanism. Similar to the approach used in Chapter 2, mechanisms underscoring the high affinity of htt for PIP and the potential role of lysine residues can be determined. For example, lysine residues in Nt17 are often acetylated, altering their charge and modifying htt-exon1's ability to associate with lipid membranes. Acetylation mimicking mutations in Nt17 can be employed to investigate their role in binding with membranes enriched with physiologically relevant amounts of PIP. By targeting these lipids, Nt17, and optimizing physiologically similar conditions to study these underlying concepts, clear mechanisms 
will be associated with htt aggregation that can potentially lead to the rationale design of strategies to target htt/PIP binding events for therapeutic purposes. Additional significance is providing extended fundamental knowledge of the membrane mediated htt aggregation.

\subsection{Exploration of PTM Crosstalk, Charged and Complex Lipid Systems, and Organelles with $\mathrm{Htt}$}

As mentioned in Chapter 2, certain PTMs are crucial modulators of htt aggregation and toxicity. ${ }^{21,22}$ The high number of overall PTMs within Nt17 implies that these modifications are essential to the functions of htt, in health, and disease ${ }^{23} \mathrm{In}$ particular, Nt17 contains numerous residues for two charge altering PTMs, acetylation and phosphorylation. ${ }^{24}$ The juxtaposition of the phosphorylation and acetylation PTMs induces crosstalk between the residues, establishing a molecular switch for modifying the dynamics of htt aggregation and function..$^{23}$ Clarifying the regulatory molecular mechanisms of these PTMs and the consequences of such modifications, is crucial for elucidating the basis of many htt functions within HD. For example, the acetylation of K6 residues in htt reverse aggregation inhibitory effects of T3 phosphorylation, due to the close proximity and stabilizing role of $\mathrm{K} 6$ in Nt17 helix formation. ${ }^{25}$ However, more detailed studies are necessary to determine the various mechanisms in which PTMs crosstalk, and their downstream effects on htt aggregation and lipid binding in vivo. 


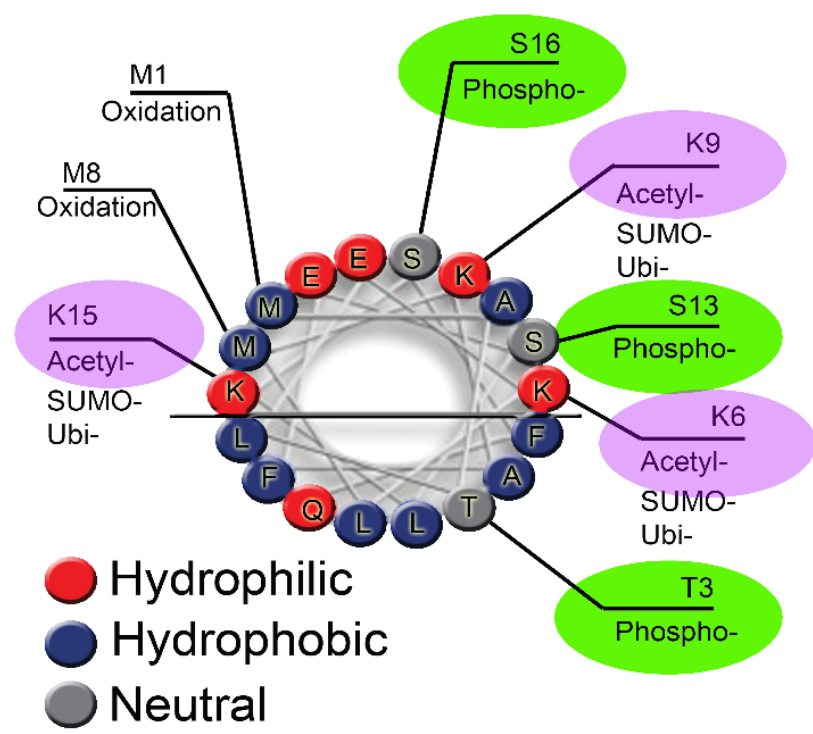

Figure 4.2: Comparison of phosphorylation and acetylation PTM sites on Nt17. Overhead view of the barrel of the Nt17 $\alpha$-helix, showing hydrophobicity of each residue (as indicated by legend) and all reported PTMs. The phosphorylation PTM sites (green) of T3, S13 and S16 are in close proximity to the acetylation PTM sites (purple) of $K 9$ and $K 6$. The center line is representative of the membrane of cells, and K6, K15 and S13 are all noticeably closer to the membrane. This suggests that selectively altering these PTMs together should have even stronger effects on lipid binding. Adapted in part with permission from Arndt, J.R. et al. The Emerging Role of the First 17 Amino Acids of Huntingtin in Huntington's Disease. Copyright 2015 Biomolecular Concepts.

There are also implications that membrane affinity influenced by phosphorylated htt is sensitized to complex, defect riddled membranes. In further expansion of the ideas outlined above, increased modification of electrostatic interactions using double and triple site phosphomimics and increasing cross talk via combinations of acetylation and phosphorylation PTMs mimics in Nt17, both serve as potential outlets for targeting specific cellular and subcellular membranes of differing composition. Additionally, phosphorylation and acetylation in tandem modify the localization of huntingtin throughout cells. ${ }^{26-31}$ This suggests that including the study of complex membranes found on cellular organelles, such as mitochondria and endoplasmic reticulum (ER), would be suitable future directions to the expansion of this study. 
Another hallmark of $\mathrm{HD}$ is altered mitochondrial morphology, with various irregularities in dissimilar cell types. ${ }^{32}$ Modified mitochondrial structures correspond to mitochondrial dysfunction in HD cells, established by numerous factors including decreased electron transport chain activity. ${ }^{32,33}$ In particular, neurons are distinguished by amplified mitochondrial fragmentation. ${ }^{34-37}$ Additionally, ER stress progresses in many neurodegenerative diseases including $H D, 32,38,39$ and it is a key influence in the deterioration of cells. ${ }^{34,40-42}$ As misfolded proteins intermingle and aggregate in the cell, toxic species arise that cause ER stress and disrupt normal cellular function. ${ }^{34}$ With a cytosolic protein such as htt, intrusion of oligomers with ER-associated degradation (ERAD) mechanisms has been elucidated in HD to be an influential process of initiating ER stress. ${ }^{43,44}$ Fundamental concepts elucidated in Chapter 2 could be further applied to see if increasing electrostatic interactions through PTMs leads to protective effects in downstream aggregation in the presence of these complex organelles; or determine if there is increased selective affinity of various PTMs within Nt17 for these membranes.

Other charged, pure lipid systems such as the anionic POPS or DOPG lipids, or mixed model lipid systems could also be studied to fine tune the effects of particular lipids found within the complex membranes of cells. Insertion depths of PTM residues on various membranes could be monitored in an attempt to shed light on particular binding mechanisms that occur, to further elucidate pre-existing findings associated with PTMs, including crosstalk between different PTMs. PTMs likely play a prominent role in htt's normal function and modify mutant htt toxicity. Understanding the complex relation between PTMs, lipid association, aggregation, and toxicity would hopefully lead to novel therapeutic strategies targeting Nt17. 


\subsection{Liquid/liquid Phase Separations, Stress Granules, and Htt Aggregation Mechanisms In Vivo.}

In Chapter 3, macromolecular crowding impacted the formation of htt aggregate species and aggregation at interfaces. In particular, large htt granules were observed under some crowding conditions that morphologically were similar to stress granules (SGs) found in cellular environments. Traditionally, isolation of molecules into organelles is attained by encompassing the organelles with lipid membranes. ${ }^{45}$ Microniches within the cell that demonstrate liquid-like qualities, including SGs, nucleoli, germ granules and paraspeckles, have been collectively labelled as biomolecular condensates ${ }^{46}$ or membraneless organelles (MLOs). ${ }^{47-49}$ MLOs are intracellular assemblies that are key to numerous fundamental mechanisms within in cell, including protein transport. ${ }^{50,51}$ Association of MLOs occurs through a process termed liquid-liquid phase separation (LLPS), a metastable de-mixing process of proteins facilitated by temporary multivalent interactions. ${ }^{52-56}$ Several diverse interactions influence LLPS that in turn, promotes formation of explicit MLOs with varying functions, as demonstrated by SGs and countless others. ${ }^{46,49,50}$ Solutions of macromolecules that endure LLPS condense into a dense phase that is similar to liquid droplets, coalescing with a dilute phase. ${ }^{57}$ The fundamental driving force of this process being the switch of macromolecule/water interactions for energetically favorable water/water interactions and macromolecule/macromolecule interactions. ${ }^{57}$ The propensity of a solution to engage in LLPS depends on numerous factors, including concentration and identities of the macromolecules involved, temperature, salt concentration, co-solutes, and volume exclusion. ${ }^{47,50,57}$ 
The cytosol in particular contains high levels of disordered proteins that are not entirely neutral. ${ }^{58}$ The formation of macroscopic puncta (or granules/bodies) in the cell from these proteins adds an additional level of complexity ${ }^{47}$ The cytoplasm and nucleoplasm of a multitude of cells are interspersed with condensates, that are disconnected from the adjacent fluid, which are dynamically established and liquefied over time. ${ }^{47,58,59}$ SGs are particularly relevant to neurodegenerative diseases, as functions associated with SG response have mutations associated with motor neuron diseases. ${ }^{45}$ Therapeutic tactics intended for hindering SG accretion have shown to reduce disease advancement in animal models of neurodegenerative diseases. ${ }^{45,60,61}$

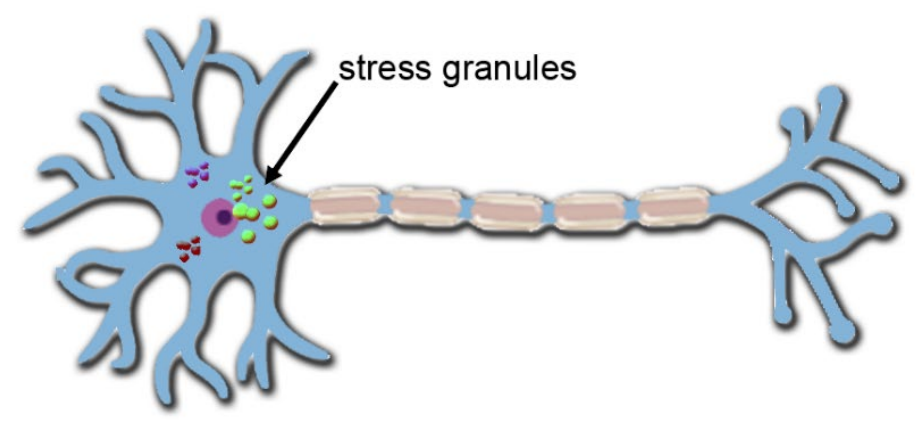

Figure 4.3: Location of stress granules (SGs). While found in numerous cellular environments, SGs can be found in close proximity to the nucleus of motor neurons. This is significant as htt also accumulates in and around the nuclei of neurons, leading to the assumption that SGs interact with and possibly affect htt aggregation. Created in part with BioRender.com

Additionally, there is significant overlap among proteins that undergo LLPs and those that aggregate in neurodegenerative diseases. ${ }^{50,62-64}$ Proteins containing intrinsically disordered regions (IDRs) stimulate LLPS yet come at the cost of being highly aggregation prone, suggesting that this overlap might be connected in disease pathogenesis. ${ }^{50,62-65}$ In vitro models of purified proteins and synthetic crowding agents 
have been explored to determine the effect of crowding on MLOs, ${ }^{47}$ though research is lacking in this area with htt. In vitro studies are essential to providing insight to the functions and qualities of MLOs on biomolecular processes, ${ }^{46,49,52-54,65-73}$ though the effect of macromolecular crowding on LLPS is rarely discussed in detail despite them being a common addition in these studies. ${ }^{47}$ Thus, macromolecular crowding influences phase separation in mixtures that would not otherwise demonstrate crowder-mediated LLPS in living cells. ${ }^{47}$

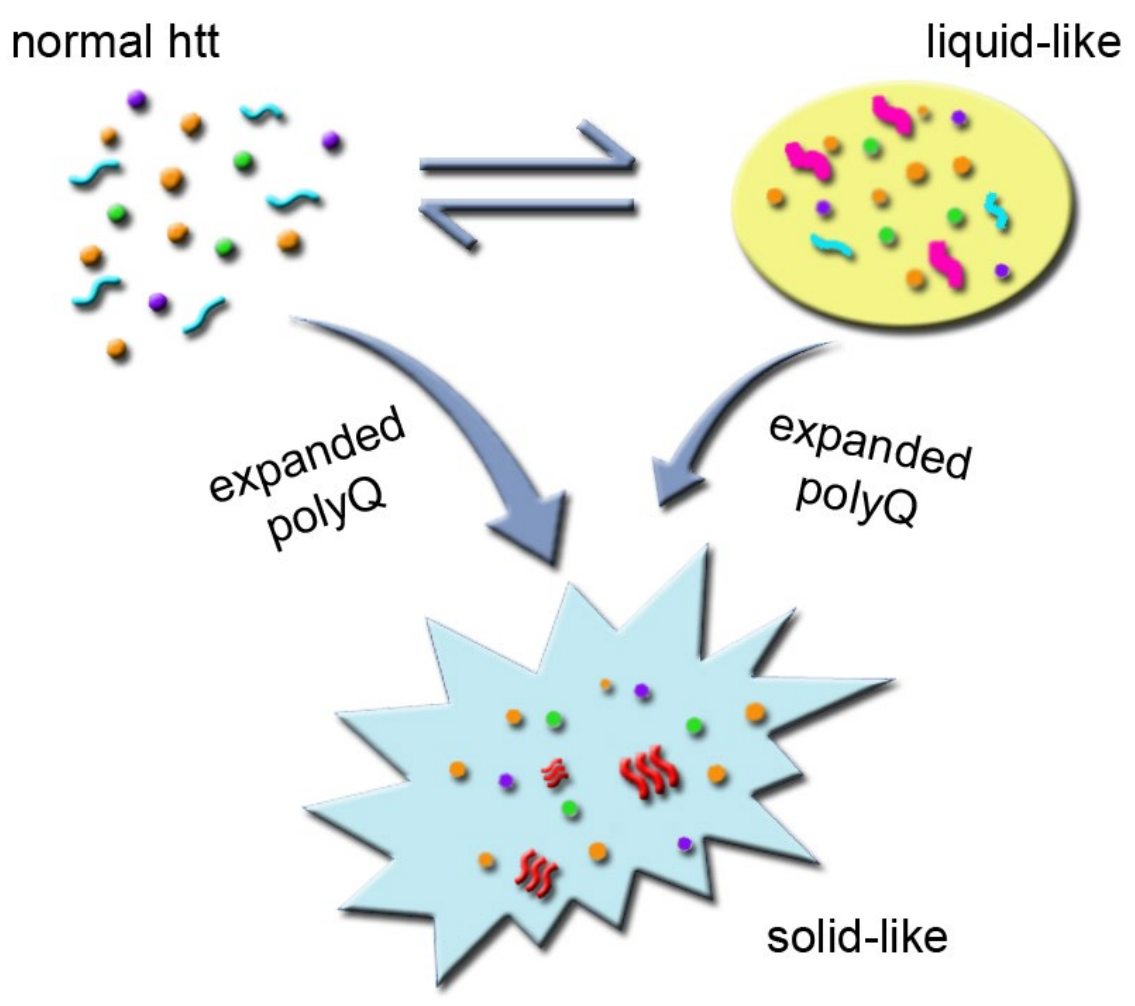

Figure 4.4: Lipid/Lipid phase separation (LLPS) of htt. Normal htt (blue, top left and right) in cellular conditions can undergo a reversible, transitional process in which aggregates (pink, top right) begin to form, but not in enough severity to form fibrillar structures. This dense collection of proteins and macromolecules (represented by green, orange and purple dots) then begin to condense into a liquid-like phase within the surrounding fluid. From here, in cases of expanded poly $Q$, the liquid phase can progress into a solid-like phase. Alternatively, this normal htt can undergo a similar process, where aggregates form and condense into solid-like structures such as fibrils (red, bottom). In this alternative pathway, the presence of expanded polyQ regions within htt can promote a direct transition to these solid-like structures. Created in part with BioRender.com 
Current understanding in the formation of MLOs such as SGs within cells, raises another possible aggregation mechanism for htt, as the aggregation mechanism of htt in vivo is still unclear. ${ }^{74} \mathrm{Htt}-$-exon1-fluorescent fusion proteins frequently accumulate into micron-sized aggregates, orders of magnitude larger than the deposits frequently analyzed in vitro. ${ }^{74}$ Ultrastructural studies allude that these aggregates contain granular and/or fibrillar substructures. ${ }^{75-77}$ Solidification of protein condensates such as SGs has also been associated to other neurodegenerative diseases such as PD and $A D$, in which they lose their liquid-like properties and begin forming amyloid fibrils. ${ }^{78,79}$ Investigation of the electrostatic interactions which influence multiple factors involved in the thermodynamic mixing of LLPS, could prove insightful to determining the threshold at which some MLOs become too dense and begin to form condensates that affect normal cellular function. In addition to defects, Nt17 has a distinct relationship with the curvature of surfaces. ${ }^{80}$ The presence of multiple complex lipid membranes and the surfaces of MLOs indicates exploring details about the fluidity, composition and curvature of these surface components could prove useful in elucidating in vivo mechanisms of htt aggregation. Together, these concepts provide motivation to determine how macromolecular crowding induces LLPS in htt and if that in turn, furthers the severity of aggregation.

\subsection{References}

(1) Choo, Y. S., Johnson, G. V. W., MacDonald, M., Detloff, P. J., and Lesort, M. (2004) Mutant huntingtin directly increases susceptibility of mitochondria to the calciuminduced permeability transition and cytochrome c release. Hum Mol Genet 13, 14071420. 
(2) Orr, A. L., Li, S., Wang, C.-E., Li, H., Wang, J., Rong, J., Xu, X., Mastroberardino, P. G., Greenamyre, J. T., and Li, X.-J. (2008) N-Terminal Mutant Huntingtin Associates with Mitochondria and Impairs Mitochondrial Trafficking. J Neurosci 28, 2783-2792.

(3) Bäuerlein, F. J. B., Saha, I., Mishra, A., Kalemanov, M., Martínez-Sánchez, A., Klein, R., Dudanova, I., Hipp, M. S., Hartl, F. U., Baumeister, W., and Fernández-Busnadiego, R. (2017) In Situ Architecture and Cellular Interactions of PolyQ Inclusions. Cell 171, 179-187.e10.

(4) Liu, K.-Y., Shyu, Y.-C., Barbaro, B. A., Lin, Y.-T., Chern, Y., Thompson, L. M., James Shen, C.-K., and Marsh, J. L. (2015) Disruption of the nuclear membrane by perinuclear inclusions of mutant huntingtin causes cell-cycle re-entry and striatal cell death in mouse and cell models of Huntington's disease. Hum Mol Genet 24, 16021616.

(5) Adegbuyiro, A., Sedighi, F., Pilkington, A. W., Groover, S., and Legleiter, J. (2017) Proteins Containing Expanded Polyglutamine Tracts and Neurodegenerative Disease. Biochemistry 56, 1199-1217.

(6) Truant, R., Atwal, R., and Burtnik, A. (2006) Hypothesis: huntingtin may function in membrane association and vesicular trafficking Biochem. Cell Biol. 84, 912-917.

(7) Kegel, K. B., Sapp, E., Yoder, J., Cuiffo, B., Sobin, L., Kim, Y. J., Qin, Z.-H., Hayden, M. R., Aronin, N., Scott, D. L., Isenberg, G., Goldmann, W. H., and DiFiglia, M. (2005) Huntingtin Associates with Acidic Phospholipids at the Plasma Membrane. J Biol Chem 280, 36464-36473.

(8) Kegel, K. B., Sapp, E., Alexander, J., Valencia, A., Reeves, P., Li, X., Masso, N., Sobin, L., Aronin, N., and DiFiglia, M. (2009) Polyglutamine expansion in huntingtin alters its interaction with phospholipids. J Neurochem 110, 1585-1597.

(9) Kegel-Gleason, K. B. (2013) Huntingtin Interactions with Membrane Phospholipids: Strategic Targets for Therapeutic Intervention? JHD 2, 239-250.

(10) Burke, K. A., Kauffman, K. J., Umbaugh, C. S., Frey, S. L., and Legleiter, J. (2013) The Interaction of Polyglutamine Peptides with Lipid Membranes Is Regulated by Flanking Sequences Associated with Huntingtin. J Biol Chem 288, 14993-15005. (11) Binder, H., and Zschörnig, O. (2002) The effect of metal cations on the phase behavior and hydration characteristics of phospholipid membranes. Chem Phys Lipids 115, 39-61.

(12) Wen, Y., Vogt, V. M., and Feigenson, G. W. (2018) Multivalent Cation-Bridged PI(4,5)P2 Clusters Form at Very Low Concentrations. Biophys J 114, 2630-2639. (13) Bradley, R. P., Slochower, D. R., Janmey, P. A., and Radhakrishnan, R. (2020) Divalent cations bind to phosphoinositides to induce ion and isomer specific propensities for nano-cluster initiation in bilayer membranes. $R$ Soc Open Sci 7. (14) Sarmento, M. J., Coutinho, A., Fedorov, A., Prieto, M., and Fernandes, F. (2014) $\mathrm{Ca} 2+$ induces $\mathrm{PI}(4,5) \mathrm{P} 2$ clusters on lipid bilayers at physiological $\mathrm{Pl}(4,5) \mathrm{P} 2$ and $\mathrm{Ca} 2+$ concentrations. BBA - Biomembranes 1838, 822-830.

(15) Redfern, D. A., and Gericke, A. (2005) pH-dependent domain formation in phosphatidylinositol polyphosphate/phosphatidylcholine mixed vesicles. J Lipid Res 46, 504-515.

(16) Al-Ramahi, I., Giridharan, S. S. P., Chen, Y.-C., Patnaik, S., Safren, N., Hasegawa, J., de Haro, M., Wagner Gee, A. K., Titus, S. A., Jeong, H., Clarke, J., Krainc, D., Zheng, W., Irvine, R. F., Barmada, S., Ferrer, M., Southall, N., Weisman, L. S., Botas, 
J., and Marugan, J. J. Inhibition of PIP4Ky ameliorates the pathological effects of mutant huntingtin protein. eLife 6.

(17) Jacobsen, J. C., Gregory, G. C., Woda, J. M., Thompson, M. N., Coser, K. R., Murthy, V., Kohane, I. S., Gusella, J. F., Seong, I. S., MacDonald, M. E., Shioda, T., and Lee, J.-M. (2011) HD CAG-correlated gene expression changes support a simple dominant gain of function. Hum Mol Genet 20, 2846-2860.

(18) Tang, T.-S., Tu, H., Chan, E. Y. W., Maximov, A., Wang, Z., Wellington, C. L., Hayden, M. R., and Bezprozvanny, I. (2003) Huntingtin and huntingtin-associated protein 1 influence neuronal calcium signaling mediated by inositol- $(1,4,5)$ triphosphate receptor type 1. Neuron 39, 227-239.

(19) Humbert, R., Bryson, E. A., Cordelières, F. P., Connors, N. C., Datta, E. R., Finkbeiner, S., and Greenberg, M. E. (2002) The IGF-1/Akt pathway is neuroprotective in Huntington's disease and involves Huntingtin phosphorylation by Akt. Dev Cell 831837.

(20) Han, B., He, K., Cai, C., Tang, Y., Yang, L., Heinemann, S. H., Hoshi, T., and Hou, S. (2016) Human EAG channels are directly modulated by PIP 2 as revealed by electrophysiological and optical interference investigations. Sci Rep 6, 23417. (21) Chaibva, M., Arndt, J. R., Valentine, S. J., and Legleiter, J. (2015) Acetylation Regulates the Interaction of Huntingtin with Lipid Membranes: Implications for Huntington Disease. Biophys J 108, 254a.

(22) Sedighi, F., Adegbuyiro, A., and Legleiter, J. (2020) SUMOylation Prevents Huntingtin Fibrillization and Localization onto Lipid Membranes. ACS Chem Neurosci 11, 328-343.

(23) Ansaloni, A., Wang, Z.-M., Jeong, J. S., Ruggeri, F. S., Dietler, G., and Lashuel, H. A. (2014) One-Pot Semisynthesis of Exon 1 of the Huntingtin Protein: New Tools for Elucidating the Role of Posttranslational Modifications in the Pathogenesis of Huntington's Disease. Angew Chem Int Ed 53, 1928-1933.

(24) Schramp, M., Hedman, A., Li, W., Tan, X., and Anderson, R. (2012) PIP Kinases from the Cell Membrane to the Nucleus, in Phosphoinositides I: Enzymes of Synthesis and Degradation (Balla, T., Wymann, M., and York, J. D., Eds.), pp 25-59.

(25) Chiki, A., DeGuire, S. M., Ruggeri, F. S., Sanfelice, D., Ansaloni, A., Wang, Z.-M., Cendrowska, U., Burai, R., Vieweg, S., Pastore, A., Dietler, G., and Lashuel, H. A. (2017) Mutant Exon1 Huntingtin Aggregation is Regulated by T3 PhosphorylationInduced Structural Changes and Crosstalk between T3 Phosphorylation and Acetylation at K6. Angew Chem Int Ed 56, 5202-5207.

(26) Rockabrand, E., Slepko, N., Pantalone, A., Nukala, V. N., Kazantsev, A., Marsh, J. L., Sullivan, P. G., Steffan, J. S., Sensi, S. L., and Thompson, L. M. (2007) The first 17 amino acids of Huntingtin modulate its sub-cellular localization, aggregation and effects on calcium homeostasis. Hum Molec Genet 16, 61-77.

(27) Thompson, L. M., Aiken, C. T., Kaltenbach, L. S., Agrawal, N., Illes, K., Khoshnan, A., Martinez-Vincente, M., Arrasate, M., O'Rourke, J. G., Khashwji, H., Lukacsovich, T., Zhu, Y.-Z., Lau, A. L., Massey, A., Hayden, M. R., Zeitlin, S. O., Finkbeiner, S., Green, K. N., LaFerla, F. M., Bates, G., Huang, L., Patterson, P. H., Lo, D. C., Cuervo, A. M., Marsh, J. L., and Steffan, J. S. (2009) IKK phosphorylates Huntingtin and targets it for degradation by the proteasome and lysosome. J Cell Biol 187, 1083-1099. 
(28) Gu, X., Greiner, E. R., Mishra, R., Kodali, R., Osmand, A., Finkbeiner, S., Steffan, J. S., Thompson, L. M., Wetzel, R., and Yang, X. W. (2009) Serines 13 and 16 Are Critical Determinants of Full-length Human Mutant Huntingtin-Induced Disease Pathogenesis in HD Mice. Neuron 64, 828-840.

(29) Atwal, R. S., Desmond, C. R., Caron, N., Maiuri, T., Xia, J., Sipione, S., and Truant, R. (2011) Kinase inhibitors modulate huntingtin cell localization and toxicity. Nat Chem Biol 7, 453-460.

(30) Ehrnhoefer, D. E., Sutton, L., and Hayden, M. R. (2011) Small changes, big impact: Posttranslational modifications and function of huntingtin in Huntington disease. Neuroscientist 17, 475-492.

(31) Saudou, F., and Humbert, S. (2016) The Biology of Huntingtin. Neuron 89, 910926.

(32) Intihar, T. A., Martinez, E. A., and Gomez-Pastor, R. (2019) Mitochondrial Dysfunction in Huntington's Disease; Interplay Between HSF1, p53 and PGC-1a Transcription Factors. Front Cell Neurosci 13, 10.

(33) Oliveira, J. M. A. (2010) Nature and cause of mitochondrial dysfunction in Huntington's disease: focusing on huntingtin and the striatum. J Neurochem 114, 1-12. (34) Shacham, T., Sharma, N., and Lederkremer, G. Z. (2019) Protein Misfolding and ER Stress in Huntington's Disease. Front Mol Biosci 6, 20.

(35) Jin, Y. N., Yu, Y. V., Gundemir, S., Jo, C., Cui, M., Tieu, K., and Johnson, G. V. W. (2013) Impaired Mitochondrial Dynamics and Nrf2 Signaling Contribute to Compromised Responses to Oxidative Stress in Striatal Cells Expressing Full-Length Mutant Huntingtin. PLOS ONE 8, e57932.

(36) Squitieri, F., Cannella, M., Sgarbi, G., Maglione, V., Falleni, A., Lenzi, P., Baracca, A., Cislaghi, G., Saft, C., Ragona, G., Russo, M. A., Thompson, L. M., Solaini, G., and Fornai, F. (2006) Severe ultrastructural mitochondrial changes in lymphoblasts homozygous for Huntington disease mutation. Mech Ageing Dev 127, 217-220. (37) Kim, J., Moody, J. P., Edgerly, C. K., Bordiuk, O. L., Cormier, K., Smith, K., Beal, M. F., and Ferrante, R. J. (2010) Mitochondrial loss, dysfunction and altered dynamics in Huntington's disease. Hum Mol Genet 19, 3919-3935.

(38) Ogen-Shtern, N., Ben David, T., and Lederkremer, G. Z. (2016) Protein aggregation and ER stress. Brain Res 1648, 658-666.

(39) Remondelli, P., and Renna, M. (2017) The Endoplasmic Reticulum Unfolded Protein Response in Neurodegenerative Disorders and Its Potential Therapeutic Significance. Front Mol Neurosci 10.

(40) Stutzbach, L. D., Xie, S. X., Naj, A. C., Albin, R., Gilman, S., Lee, V. M. Y., Trojanowski, J. Q., Devlin, B., Schellenberg, G. D., and PSP Genetics Study Group. (2013) The unfolded protein response is activated in disease-affected brain regions in progressive supranuclear palsy and Alzheimer's disease. Acta Neuropathol Commun 1, 31.

(41) Heman-Ackah, S. M., Manzano, R., Hoozemans, J. J., Scheper, W., Flynn, R., Haerty, W., Cowley, S. A., Bassett, A. R., and Wood, M. J. (2017) Alpha-synuclein induces the unfolded protein response in Parkinson's disease SNCA triplication iPSCderived neurons. Hum Mol Genet 26, 4441-4450. 
(42) Vidal, R., Caballero, B., Couve, A., and Hetz, C. (2011) Converging Pathways in the Occurrence of Endoplasmic Reticulum (ER) Stress in Huntingtons Disease. CMM $11,1-12$.

(43) Shorter, J., and Lindquist, S. (2005) Prions as adaptive conduits of memory and inheritance. Nat Rev Genet 6, 435-450.

(44) Leitman, J., Ulrich Hartl, F., and Lederkremer, G. Z. (2013) Soluble forms of polyQexpanded huntingtin rather than large aggregates cause endoplasmic reticulum stress.

Nat Commun 4, 2753.

(45) Wolozin, B., and Ivanov, P. (2019) Stress granules and neurodegeneration. Nat Rev Neurosci 20, 649-666.

(46) Banani, S. F., Lee, H. O., Hyman, A. A., and Rosen, M. K. (2017) Biomolecular condensates: organizers of cellular biochemistry. Nat Rev Mol Cell Biol 18, 285-298.

(47) André, A. A. M., and Spruijt, E. (2020) Liquid-Liquid Phase Separation in Crowded Environments. IJMS 21, 5908.

(48) Aumiller, W. M., and Keating, C. D. (2017) Experimental models for dynamic compartmentalization of biomolecules in liquid organelles: Reversible formation and partitioning in aqueous biphasic systems. Adv Colloid Interface Sci 239, 75-87.

(49) Mitrea, D. M., and Kriwacki, R. W. (2016) Phase separation in biology; functional organization of a higher order. Cell Commun Signal 14, 1.

(50) Babinchak, W. M., and Surewicz, W. K. (2020) Liquid-Liquid Phase Separation and Its Mechanistic Role in Pathological Protein Aggregation. J Mol Biol 432, 1910-1925.

(51) Pancsa, R., Schad, E., Tantos, A., and Tompa, P. (2019) Emergent functions of proteins in non-stoichiometric supramolecular assemblies. BBA - Proteins and Proteomics 1867, 970-979.

(52) Treeck, B. V., and Parker, R. (2018) Emerging Roles for Intermolecular RNA-RNA Interactions in RNP Assemblies. Cell 174, 791-802.

(53) Banani, S. F., Rice, A. M., Peeples, W. B., Lin, Y., Jain, S., Parker, R., and Rosen, M. K. (2016) Compositional Control of Phase-Separated Cellular Bodies. Cell 166, 651663.

(54) Shin, Y., and Brangwynne, C. P. (2017) Liquid phase condensation in cell physiology and disease. Science 357.

(55) Gomes, E., and Shorter, J. (2019) The molecular language of membraneless organelles. J Biol Chem 294, 7115-7127.

(56) Wang, J., Choi, J.-M., Holehouse, A. S., Lee, H. O., Zhang, X., Jahnel, M., Maharana, S., Lemaitre, R., Pozniakovsky, A., Drechsel, D., Poser, I., Pappu, R. V., Alberti, S., and Hyman, A. A. (2018) A Molecular Grammar Governing the Driving Forces for Phase Separation of Prion-like RNA Binding Proteins. Cell 174, 688-699.e16. (57) Alberti, S., Gladfelter, A., and Mittag, T. (2019) Considerations and Challenges in Studying Liquid-Liquid Phase Separation and Biomolecular Condensates. Cell 176, 419-434.

(58) Hyman, A. A., and Brangwynne, C. P. (2011) Beyond stereospecificity: liquids and mesoscale organization of cytoplasm. Dev Cell 21, 14-16.

(59) Brangwynne, C. P., Eckmann, C. R., Courson, D. S., Rybarska, A., Hoege, C., Gharakhani, J., Jülicher, F., and Hyman, A. A. (2009) Germline P granules are liquid droplets that localize by controlled dissolution/condensation. Science 324, 1729-1732. 
(60) Kim, H.-J., Raphael, A. R., LaDow, E. S., McGurk, L., Weber, R., Trojanowski, J. Q., Lee, V. M.-Y., Finkbeiner, S., Gitler, A. D., and Bonini, N. M. (2014) Therapeutic modulation of elF2a-phosphorylation rescues TDP-43 toxicity in amyotrophic lateral sclerosis disease models. Nat Genet 46, 152-160.

(61) Radford, H., Moreno, J. A., Verity, N., Halliday, M., and Mallucci, G. R. (2015) PERK inhibition prevents tau-mediated neurodegeneration in a mouse model of frontotemporal dementia. Acta Neuropathol 130, 633-642.

(62) Aguzzi, A., and Altmeyer, M. (2016) Phase Separation: Linking Cellular Compartmentalization to Disease. Trend Cell Biol 26, 547-558.

(63) Nedelsky, N. B., and Taylor, J. P. (2019) Bridging biophysics and neurology: aberrant phase transitions in neurodegenerative disease. Nat Rev Neurol 15, 272-286. (64) Ryan, V. H., and Fawzi, N. L. (2019) Physiological, Pathological, and Targetable Membraneless Organelles in Neurons. Trend Neurosci 42, 693-708.

(65) Boeynaems, S., Alberti, S., Fawzi, N. L., Mittag, T., Polymenidou, M., Rousseau, F., Schymkowitz, J., Shorter, J., Wolozin, B., Bosch, L. V. D., Tompa, P., and Fuxreiter, M. (2018) Protein Phase Separation: A New Phase in Cell Biology. Trend Cell Biol 28, 420-435.

(66) Mitrea, D. M., Chandra, B., Ferrolino, M. C., Gibbs, E. B., Tolbert, M., White, M. R., and Kriwacki, R. W. (2018) Methods for Physical Characterization of Phase-Separated Bodies and Membrane-less Organelles. J Mol Biol 430, 4773-4805.

(67) Chong, P. A., Vernon, R. M., and Forman-Kay, J. D. (2018) RGG/RG Motif Regions in RNA Binding and Phase Separation. J Mol Biol 430, 4650-4665.

(68) Bratek-Skicki, A., Pancsa, R., Meszaros, B., Lindt, J. V., and Tompa, P. (2020) A guide to regulation of the formation of biomolecular condensates. The FEBS Journal 287, 1924-1935.

(69) Alberti, S. (2017) The wisdom of crowds: regulating cell function through condensed states of living matter. J Cell Sci 130, 2789-2796.

(70) Zaslavsky, B. Y., and Uversky, V. N. (2018) In Aqua Veritas: The Indispensable yet Mostly Ignored Role of Water in Phase Separation and Membrane-less Organelles. Biochemistry 57, 2437-2451.

(71) Nakashima, K. K., Vibhute, M. A., and Spruijt, E. (2019) Biomolecular Chemistry in Liquid Phase Separated Compartments. Front Mol Biosci 6, 21.

(72) Uversky, V. N. (2017) Intrinsically disordered proteins in overcrowded milieu: Membrane-less organelles, phase separation, and intrinsic disorder. Curr Opin Struct Biol 44, 18-30.

(73) Ambadipudi, S., Biernat, J., Riedel, D., Mandelkow, E., and Zweckstetter, M. (2017) Liquid-liquid phase separation of the microtubule-binding repeats of the Alzheimerrelated protein Tau. Nat Commun 8, 275.

(74) Peskett, T. R., Rau, F., O'Driscoll, J., Patani, R., Lowe, A. R., and Saibil, H. R. (2018) A Liquid to Solid Phase Transition Underlying Pathological Huntingtin Exon1 Aggregation. Mol Cell 70, 588-601.e6.

(75) DiFiglia, M., Sapp, E., Chase, K. O., Davies, S. W., Bates, G. P., Vonsattel, J. P., and Aronin, N. (1997) Aggregation of Huntingtin in Neuronal Intranuclear Inclusions and Dystrophic Neurites in Brain. Science 277, 1990-1993.

(76) Legleiter, J., Mitchell, E., Lotz, G. P., Sapp, E., Ng, C., DiFiglia, M., Thompson, L. M., and Muchowski, P. J. (2010) Mutant Huntingtin Fragments Form Oligomers in a 
Polyglutamine Length-dependent Manner in Vitro and in Vivo. J Biol Chem 285, 1477714790.

(77) Scherzinger, E., Lurz, R., Turmaine, M., Mangiarini, L., Hollenbach, B., Hasenbank, R., Bates, G. P., Davies, S. W., Lehrach, H., and Wanker, E. E. (1997) HuntingtinEncoded Polyglutamine Expansions Form Amyloid-like Protein Aggregates In Vitro and In Vivo. Cell 90, 549-558.

(78) Molliex, A., Temirov, J., Lee, J., Coughlin, M., Kanagaraj, A. P., Kim, H. J., Mittag, T., and Taylor, J. P. (2015) Phase Separation by Low Complexity Domains Promotes Stress Granule Assembly and Drives Pathological Fibrillization. Cell 163, 123-133. (79) Lin, Y., Protter, D. S. W., Rosen, M. K., and Parker, R. (2015) Formation and Maturation of Phase-Separated Liquid Droplets by RNA-Binding Proteins. Mol Cell 60, 208-219.

(80) Chaibva, M., Burke, K. A., and Legleiter, J. (2014) Curvature Enhances Binding and Aggregation of Huntingtin at Lipid Membranes. Biochemistry 53, 2355-2365. 


\title{
Appendix: Huntingtin Protein Purification and Experimentation with Macromolecular Crowders: An Innovative Undergraduate Biochemistry Experiment
}

\begin{abstract}
Traditional undergraduate biochemical laboratory curricula are confronted with multitudinous challenges in improving student learning outcomes due to limitations with time, increased student enrollment, and high-cost laboratory resources. However, with the advent of cost-effective instrumentation and incorporation of creative approaches to circumvent inflexible undergraduate schedules, it is now possible to overcome these challenges. Thus, we have designed an interdisciplinary, medically relevant laboratory experiment intended to provide undergraduate students with enhanced pedagogical training through guided- and independent-based inquiry. This experiment seeks to mediate limitations common to traditional biochemical experimentation using high-level instrumentation in tandem with graduate-level student engagement.
\end{abstract}

\section{A1. Introduction}

Science, technology, engineering, and mathematic (STEM) educational programs have seen an increase in majors, including subdivisions such as biochemistry, bioinorganic chemistry, bioorganic chemistry, biophysical chemistry, agriculture, and food science. ${ }^{1-3}$ Rigorous coursework and laboratories, along with undergraduate research experiences, are essential for STEM majors; however, not all students can participate in undergraduate research. Thus, students do not obtain the crucial training or engaged learning needed for upper-level educational programs or STEM careers. The need for undergraduate laboratory experiments that provide relevant graduate-level experiences is becoming increasingly necessary. Available 
biochemistry laboratories have seen very little graduate-level experimentation because laboratory design tends to follow an expository style format where students acquire very limited and superficial knowledge through passive completion of a "cookbook" protocol. ${ }^{4}$ This is due in part because most undergraduate scientific laboratory experiments have limitations with both equipment and time. ${ }^{4-8}$

Typical biochemistry laboratory courses consist of a two-semester model where the first semester focuses on acquiring basic biochemical skills, including proper pipetting techniques, preparation of solutions and buffers, and familiarization with basic instrumentation such as electrophoresis, colorimetric ultraviolet-visible spectroscopy and size exclusion chromatography. The second semester centers on higher-level biochemical techniques for the analysis of biological species and processes, including cell growth and protein expression. Emphasis is applied on providing knowledge of biochemical techniques from a practical perspective and developing critical thinking skills to prepare graduates. This model, although established and convenient, is problematic in that the time constraints of most undergraduate biochemistry laboratories allow for unoriginal applications that do not facilitate intellectual growth through critical thinking skills development that is provided via research-based experiences. Thus, these educational labs lack the veracities associated with higher level biochemical employment or education. The idea of straying from a traditional learning format by changing experimentation to a more research-based approach has been well received. ${ }^{4,9-11}$

Cost effective availability of biological instrumentation has made it possible to add a more advanced component to biochemical laboratory experiments. Refrigerated 
centrifuges, microplate reader spectrophotometers, digital incubators, and specialized chromatography columns are now available and could provide valuable experiences when transitioning to graduate level education or industry. For example, protein expression and purification in undergraduate laboratories has been successful with diverse recombinant proteins in Escherichia Coli (E. Coli). Opportunities for experimental modifications exist within the protein expression and purification processes $^{9-12}$ and have been demonstrated with versatility in chromatography. ${ }^{11}$ Despite these modifications, without a change in the style of learning, students will not be able to reap the benefits of a graduate level experience.

We have designed an undergraduate biochemistry laboratory experiment involving the huntingtin protein (htt) that incorporates guided and inquiry-based experimentation with emphasis on instrumentation and graduate-level participation. This experiment models other independent graduate level work being conducted but has been adapted to accommodate the inflexible undergraduate timeline. The experiment focuses on immersion into the graduate school environment coupled with the use of advanced instrumentation, which will provide excellent undergraduate learning opportunities that can be expanded and are markedly useful when moving forward in a scientific career. Further, interest amongst the students will persist and will encourage independent research on the topic by using a popular and medically relevant protein, in contrast to traditional enzymatic based biochemistry experiments. ${ }^{9,12-14}$

\section{A2. Background}

The primary genetic cause of several neurodegenerative diseases is the expansion of trinucleotide repeats to a greater number than what occurs in the naturally 
occurring stable gene..$^{15-17}$ Trinucleotide repeat disorders (TRDs) result from an abnormal expansion of repetitive codon sequences within a gene and primarily affect the nervous system. A subclassification of TRDs consist of a group of 10 disorders in which the codon CAG, which encodes for the amino acid glutamine $(\mathrm{Q})$, is repeated and results in an expanded polyglutamine (polyQ) tract within the translated protein. This subset of TRDs is commonly referred to as CAG repeat disorders or polyQ diseases. The 10 identified CAG repeat disorders include Huntington's Disease (HD), ${ }^{18}$ dentatorubal-pallidoluysian atrophy (DRPLA or Haw River Syndrome), ${ }^{19}$ the spinocerebellar ataxias, type 1 (SCA-1), type 2 (SCA-2), type 3 (SCA-3 or MachadoJoseph disease), type 6 (SCA-6), type 7 (SCA-7), type 12 (SCA-12), type 17 (SCA$17)^{20-26}$ and spinobulbar muscular atrophy (SBMA or Kennedy's disease). ${ }^{27}$

Generally, expansion of polyQ beyond a critical threshold is required for disease. Onset of symptoms and age are strongly correlated to polyQ length. In HD, polyQ repeat lengths of approximately $35 \mathrm{in} \mathrm{htt} \mathrm{are} \mathrm{considered} \mathrm{the} \mathrm{critical} \mathrm{threshold,} \mathrm{with}$ increased severity as the repeat length increases. ${ }^{18,28-31} \mathrm{~A}$ key hallmark of the aforementioned expanded polyQ diseases is the accumulation of abnormal proteins into proteinaceous deposits. These deposits are predominately comprised of aggregate species termed amyloid that are defined by a fibrillar morphology and a cross $\beta$-sheet structure. In particular, polyQ expansion promotes htt's aggregation into fibrils. ${ }^{32}$ However, multiple aggregation pathways exist, involving a variety of other aggregate species, including oligomers, annular structures, and amorphous aggregates. ${ }^{15,32,33}$ Within the literature, htt oligomers ${ }^{34-36}$ and fibrils ${ }^{37,38}$ have been classified as toxic entities. 
The six-week experiment outlined demonstrates the $E$. Coli based expression and purification of a GST-htt-exon1 fusion protein with a disease length polyQ domain. Cleaving the GST induces aggregation that can be tracked kinetically by a fluorescence-based aggregation assay. Students used this assay to determine the impact of variety of factors on htt aggregation. Specifically, to test if mimicking the crowded environment of the cytosol with macromolecular crowders impacted htt aggregation. Emphasis was placed on facilitation of advanced critical thinking when designing the independent experiment, graduate level presentations, and manuscript writing. Collaboration and feedback was provided throughout the weekly experiments, including personalized feedback during preliminary oral presentations.

\section{A3. Overview of Experiment}

In this six-week experiment, students completed the weekly laboratory work in a large group, 10-15 students per laboratory session, with a total of 22-30 students completing the experiment each semester. Smaller sub-groups were generated and rotated based on weekly tasks (Table A1), which allowed for participation in all the various components of the experimental procedure.

Similar to graduate level education or STEM careers, students completed both oral and written presentations of their scientific findings. Students were asked to focus on providing a professional presentation that could be delivered at a scientific conference. They were also expected to provide weekly written records along with a final manuscript that replicated the publication style of a peer-reviewed scientific journal; student manuscripts were in the style of the American Chemical Society (ACS) Biochemistry journal. 
Week 1: Several inoculations of $E$. coli with the vector to express a GST-htt-exon1 fusion protein were generated to demonstrate various stages of protein expression. The starting times of these inoculations were offset to allow students to participate in the entire process, allowing the entire process to fit within a typical three-hour laboratory period.

Students alternate between stations to gain experience with each step of E.coli growth and protein expression, including the preparation of growth media and autoclave sterilization techniques. Students set up an overnight inoculation of a strain of $E$. Coli containing the plasmid for the GST-htt fusion protein, prepare fresh media with ampicillin, and continue through the protocol with a previously prepared overnight culture. Protein expression is induced using isopropyl- $\beta$-D-thiogalactoside (IPTG) and cell harvesting is demonstrated; however, protein expression and cell harvesting of the offset inoculation was completed outside of the lab period.

Week 2: Alternating stations, students prepare buffers needed for purification, suspend cell pellets, and perform protein extraction by lysing cells with lysozyme and sonication. Using small-scale glutathione (GST) affinity centrifugation columns, each student participates in the purification process. Additionally, assays to measure protein concentration and dialysis techniques are demonstrated.

Week 3: Use of a microplate reader spectrophotometer and plate organization is discussed to prepare students for the following week's experiment (Week 4). Students then participate in an excel workshop that focuses on scientific data analysis, which students will complete independently during Week 4, and include in both their oral presentation and manuscript. 
Week 4: Students complete the required aggregation assays without significant guidance from the instructor; minimal assistance is key to ensure students are collaborating and developing metacognition skills. Although the impact of macromolecular crowding on htt aggregation was examined here, there are several experimental adaptations available.

Week 5/6: Students combine hypotheses, testing procedures, observations, results and conclusions into a scientific manuscript that replicates the publication style of a biochemical scientific journal. Students also presented their findings as if at a scientific conference, allowing early opportunities for students to receive positive feedback on how to present scientific information in various media forms when preparing for scientific careers. 
Table A1: Devised Solutions to Traditional Experimental Setbacks

\begin{tabular}{|c|c|c|}
\hline Weekly Breakdown & $\begin{array}{l}\text { Traditional } \\
\text { Setback }\end{array}$ & Solution \\
\hline Week 1: Protein Expression in E. coli & $\begin{array}{l}\text { Instrument } \\
\text { availability, time } \\
\text { management }\end{array}$ & $\begin{array}{l}\text { E. coli are staggered at various stages of growth for } \\
\text { protein expression. } \\
\text { Students are distributed into groups to gain } \\
\text { experience with all aspects of these processes. }\end{array}$ \\
\hline Week 2: Protein Purification & $\begin{array}{l}\text { Instrument } \\
\text { availability, time } \\
\text { management }\end{array}$ & $\begin{array}{l}\text { Small scale purification methods enable many } \\
\text { students to actively participate in protein purification. }\end{array}$ \\
\hline Week 3: Excel Workshop & $\begin{array}{l}\text { Limited } \\
\text { knowledge of } \\
\text { Excel, specifically } \\
\text { with advanced } \\
\text { data analysis } \\
\end{array}$ & $\begin{array}{l}\text { The Excel workshop focuses on scientific data } \\
\text { analysis, and its presentation in both oral and } \\
\text { manuscript forms. } \\
\text { Students utilize mock data with open discussions } \\
\text { encouraged. }\end{array}$ \\
\hline Week 4: Kinetic Experiment & $\begin{array}{l}\text { Lack of } \\
\text { independent } \\
\text { laboratory } \\
\text { investigations } \\
\text { that require } \\
\text { advanced } \\
\text { scientific design } \\
\text { and assessment }\end{array}$ & $\begin{array}{l}\text { Students design an experiment testing various } \\
\text { conditions based on previously learned techniques, } \\
\text { specifically with macromolecular crowders. } \\
\text { Minimal supervision is provided as students are } \\
\text { responsible for experimental setup and execution. }\end{array}$ \\
\hline $\begin{array}{l}\text { Weeks } 5 \text { \& 6: Professional Oral } \\
\text { Presentations and Scientific Reporting }\end{array}$ & $\begin{array}{l}\text { Limited } \\
\text { professional, data } \\
\text { centered public } \\
\text { speaking } \\
\text { opportunities, } \\
\text { significant } \\
\text { deficiencies in } \\
\text { scientific writing }\end{array}$ & $\begin{array}{l}\text { Students drafted and presented their analyzed data } \\
\text { over a two-week period. Emphasis was placed on } \\
\text { providing presentations that could be delivered at } \\
\text { professional scientific conferences. } \\
\text { Weekly notebooks were combined into a scientific } \\
\text { manuscript that replicates the publication style of a } \\
\text { scientific journal. }\end{array}$ \\
\hline
\end{tabular}

\section{A4. Hazards}

Bradford reagent, hydrochloric acid $(\mathrm{HCl})$, and sodium hydroxide $(\mathrm{NaOH})$ can cause burns and are corrosive. Sodium phosphates can irritate the nose and throat when inhaled and could result in respiratory difficulties; sodium phosphates can also pass through the skin and irritate the eyes. Glycerol can be harmful if swallowed. Ampicillin may cause allergic reactions. Trizma base, isopropyl- $\beta-D-$ thiogalactoside (IPTG), ethylenediaminetetraacetic acid (EDTA), yeast and tryptone may cause skin and eye irritation and may be harmful if swallowed or inhaled. Thioflavin $\mathrm{T}$ is corrosive and can cause burns, serious eye irritation and targets respiratory 
system. Huntington protein and all related waste is classified as Biological Safety Level -1 (BSL-1) and should be treated with a 10\% bleach solution and autoclaved prior to disposal.

\section{A5. Results and Discussion}

This experiment can be utilized in a biochemistry or biochemistry-related laboratory course where students have been introduced to protein and kinetic topics in previous coursework. The experiment requires four three-hour laboratory sessions with two additional three-hour sessions for oral presentations as outlined above.

Typical student results obtained during the initial three laboratory sessions (Weeks 1-3) included an average absorbance value between $0.500-0.700$ at $600 \mathrm{~nm}$ for the optical density of the larger batch broth cultures. Following purification during Week 2 of experimentation, students generally obtained approximately $10-12 \mathrm{~mL}$ of $0.2-0.4$ $\mathrm{mg} / \mathrm{mL}$ of htt. If the concentration of the protein fractions alone was greater than $20 \mu \mathrm{M}$, neat buffer was used to dilute the sample until the concentration was below $20 \mu \mathrm{M}$. This was to ensure minimal htt loss due to premature aggregation during dialysis and provide a sufficient quantity of diluted htt to generate mock data. During Week 3 , students completed an excel data analysis using mock data. In the final week of experimentation (Week 4), students determined the concentration of htt after dialysis with typical concentrations ranging from $2-10 \mu \mathrm{M}$ depending on the success of the purification. Students also developed a plate layout for the three macromolecular crowders used, Dextran, Ficoll, and polyethylene glycol (PEG). A final concentration of each macromolecular crowder at either $100 \mathrm{mg} / \mathrm{mL}$ or $50 \mathrm{mg} / \mathrm{mL}$ in $100 \mu \mathrm{L}$ (the volume in each well) was achieved while still accounting for the final target concentration of htt (10 
$\mu \mathrm{M})$. Crowder volumes for these target concentrations were determined from a dilution scheme by the students using previously prepared stocks.

A Thioflavin T (ThT) assay was used to assess fibril formation of htt incubated with the three macromolecular crowders. Students were assigned final concentrations of either $100 \mathrm{mg} / \mathrm{ml}$ or $50 \mathrm{mg} / \mathrm{ml}$ of the macromolecular crowders with the assay being completed in triplicate. Figure A1 demonstrates student obtained ThT assay data of htt in the presence and absence of crowders. Students assessed trends in fibril formation by the location of each signal in relation to htt alone. A signal higher than the htt control (no crowders) indicated enhancement of fibril formation. Polyethylene glycol (PEG) at both concentrations produced a diminished signal relative to control, indicating inhibition of fibril formation.

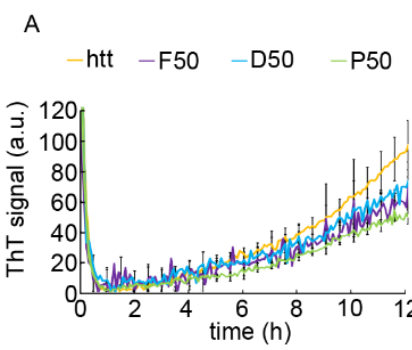

B

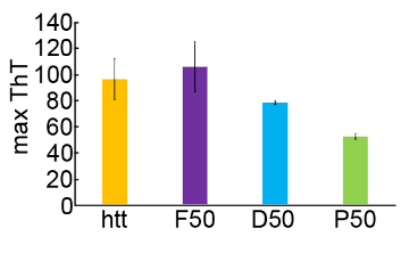

C $-\mathrm{htt}-\mathrm{F} 100-\mathrm{D} 100-\mathrm{P} 100$

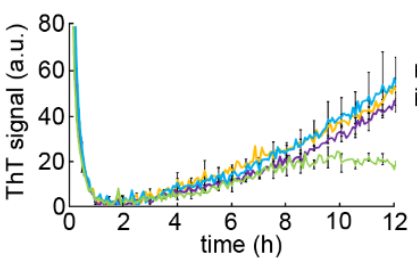

D

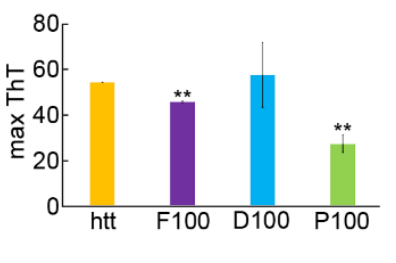

Figure A1: Representation of student obtained data during experiment. Student data with $10 \mu \mathrm{M}$ htt in the presence and absence of Ficoll, Dextran and PEG at (A) $50 \mathrm{mg} / \mathrm{mL}$ and (C) 100 $\mathrm{mg} / \mathrm{mL}$. Quantification of the maximum ThT fluorescent signal observed for Ficoll, Dextran and PEG at (B) $50 \mathrm{mg} / \mathrm{mL}$ and (D) $100 \mathrm{mg} / \mathrm{mL}$ with respect to htt alone is present. Error bars represent SEM with * corresponding to $p<0.01$ and ${ }^{* *}$ corresponds to $p<0.05$.

In addition to traditional weekly laboratory notebooks, students were assessed by the oral presentations and final manuscripts. For the laboratory notebooks, sections such as the introduction to the experiment, experimental objectives, materials and methods, results and discussion, conclusion and laboratory questions were requested 
each week. The laboratory questions as well as the experiment objectives section in the written pre-laboratory assignment were key pieces for evaluating basic knowledge of the pedagogical goals prior to completing the weekly experiments. Post-laboratory results, discussion, and conclusion sections judged the level the students understood the weekly experimental goals, identifying what information would be relevant to continue for the following week's task, and demonstrated if students were thinking critically about what to include for their manuscript. Students scored exceptionally well for the laboratory notebooks, with an average grade of $91 \%$. High scores are indicative that the pedagogical goals of each week were effectively communicated and achieved.

The oral presentations following the completion of week \#4 were used as a measure of assessment for the combination of the student's overall understanding of the experiment, professional speaking, and communication skills. Prior to the presentations, students were encouraged to follow guidelines with regards to the background information, experimental procedures, results, and conclusions. It was stressed that focus should be placed on the results, what they mean and why they were significant to the broader scientific community. Current literature and proper identification of the overall goal were also critically evaluated. Proper referencing, slide aesthetics and knowledge during the question sessions from peers and instructors were also provided as guidelines and judged to help create an immersive, conference-style setting. Again, students scored well for the oral presentations, with an average grade of $84 \%$. For most of the students, this style of evaluation and experience was their first opportunity within the program, adding a much-needed component for an upper level undergraduate course. 
Upon completion of the oral presentations, students submit a manuscript of their results written in Biochemistry format. A template for the manuscript is provided, and students are expected to use the information from their laboratory notebooks and feedback from their presentations. Students were presented with a list of appropriate sections, suggestions of what to include in each section, and were encouraged to read literature in similar areas of study and format. Deeper understanding of the ThT results, htt aggregation and fibril structure, and relation of the experiment to real world situations were expected to be elucidated. Determining if results were significant through statistics and areas for improvement were also emphasized strongly. The average grade for the manuscripts was $77 \%$, again suggestive that students were retaining the knowledge learned and effectively using critical thinking skills to structure the manuscript. This was the first experience with writing a publication-type document for students within the major, so a learning curve was to be expected.

Overall, student evaluations revealed that $79 \%$ of students enjoyed the experiment. Students responses also indicated increased satisfaction with the hands-on nature of the experiment, use of multiple lab spaces, newly gained knowledge of the preparation required for larger-scale experimentation, and group collaboration. Students also indicated the significant usefulness of learning upper-level techniques and instrumentation, including spin GST-affinity based column chromatography, and LC GST-affinity based column chromatography, large scale recombinant protein expression and cell lysis. Specific student comments encompassing the entirety of the experiment included "we were able to take part in all the steps without using multiple lab days", "the excel lab taught functions that were useful for data analysis and helpful for future 
classes", "that we actually learned how to generate complicated graphs we see in papers" and "we finally had a coursework lab that incorporates techniques used in real graduate level labs. It gave the lab group a sense of autonomy and responsibility in dividing and conquering work."

Conversation and positive encouragement were fostered throughout each week of the experiment as students encounter common experimental issues or struggle to envision the experimental goals. This is especially critical for students who have had no exposure to undergraduate research or have been conditioned for traditional "cookbook" protocols that are expected to work with little error. Additionally, early communication between student and instructor helps to bridge the guided laboratory structure to the desired independent inquiry-based format. Through these activities' students gained important knowledge in data analysis and the use of sophisticated scientific instrumentation, acquired insight into upper-level biochemical experimentation, and have a better understanding of real-world issues currently faced in biochemistry and biochemistry related fields.

\section{A6. Summary}

When compared to traditional biochemical experiments involving protein expression and purification, this experiment allowed for an atypical, interesting, and impactful protein to impart some new life into a curriculum that is steadily becoming stale and less relevant for students seeking STEM careers. Additionally, these modified protein expression, purification, and experimental protocols successfully demonstrated their innovative use by circumventing the time-constraints typically plaguing an undergraduate laboratory course along with allowing for cost effective use of equipment 
and materials. Experimental focus on improving communication and critical thinking skills are both useful and necessary for career paths in STEM. Students also have a better understanding of a biologically relevant protein and its role in $\mathrm{HD}$, along with how the efficacy of potential treatments are evaluated in disease prevention.

\section{A7. References}

(1) Biochemists and Biophysicists : Occupational Outlook Handbook: : U.S. Bureau of Labor Statistics.

(2) (2016, March 20) Stem Majors on the Rise as Humanities Decline Across the Country. Emsi.

(3) Martin-Hansen, L. (2018) Examining ways to meaningfully support students in STEM. Intl J STEM Educ 5, 53.

(4) Domin, D. S. (1999) A Review of Laboratory Instruction Styles. J Chem Educ 76, 543.

(5) Mordacq, J., Drane, D., Swarat, S., and Lo, S. (2017) Research and Teaching: Development of Course-Based Undergraduate Research Experiences Using a DesignBased Approach. J Coll Sci Teach 46.

(6) Weaver, G. C., Russell, C. B., and Wink, D. J. (2008) Inquiry-based and researchbased laboratory pedagogies in undergraduate science. Nat Chem Biol 4, 577-580.

(7) Brew, A., and Saunders, C. (2020) Making sense of research-based learning in teacher education. Teach Teach Educ 87, 102935.

(8) Sales, J., Comeau, D., Liddle, K., and Khanna, N. (2006) Bridging the Gap: A Research-Based Approach for Teaching Interdisciplinary. Sociology.

(9) Craig, P. A. (1999) A Project-Oriented Biochemistry Laboratory Course, J Chem Educ 76, 8, 1130.

(10) Maza, J. C., Villa, J. K., Landino, L. M., and Young, D. D. (2016) Utilizing Unnatural Amino Acids To Illustrate Protein Structure- Function Relationships: An Experiment Designed for an Undergraduate Biochemistry Laboratory. J Chem Educ, 5.

(11) Peterson, M. J., Snyder, W. K., Westerman, S., and McFarland, B. J. (2011) Preparative Protein Production from Inclusion Bodies and Crystallization: A SevenWeek Biochemistry Sequence. J Chem Educ, 4.

(12) MacDonald, G. (2008) Teaching Protein Purification and Characterization Techniques. A Student-Initiated, Project-Oriented Biochemistry Laboratory Course. $J$ Chem Educ 85, 1250.

(13) Deal, S. T., and Hurst, M. O. (1997) Utilizing Isolation, Purification, and Characterization of Enzymes as Project-Oriented Labs for Undergraduate Biochemistry. J Chem Educ 74, 241.

(14) Markwell, J. (1993) Focusing on a single enzyme: An undergraduate biochemistry laboratory course supported by the NSF. J Chem Educ 70, 1018.

(15) Adegbuyiro, A., Sedighi, F., Pilkington, A. W., Groover, S., and Legleiter, J. (2017) Proteins Containing Expanded Polyglutamine Tracts and Neurodegenerative Disease. Biochemistry 56, 1199-1217. 
(16) La Spada, A. R., and Taylor, J. P. (2010) Repeat expansion disease: Progress and puzzles in disease pathogenesis. Nat Rev Genet 11, 247-258.

(17) Budworth, H., and McMurray, C. T. (2013) A Brief History of Triplet Repeat Diseases. Methods Mol Biol 1010, 3-17.

(18) Macdonald, M. (1993) A novel gene containing a trinucleotide repeat that is expanded and unstable on Huntington's disease chromosomes. Cell 72, 971-983.

(19) Miyashita, T., Nagao, K., Ohmi, K., Yanagisawa, H., Okamura-Oho, Y., and Yamada, M. (1998) Intracellular Aggregate Formation of Dentatorubral-Pallidoluysian Atrophy (DRPLA) Protein with the Extended Polyglutamine. Biochem Biophys Res Co 249, 96-102.

(20) Orr, H. T., Chung, M., Banfi, S., Kwiatkowski, T. J., Servadio, A., Beaudet, A. L., McCall, A. E., Duvick, L. A., Ranum, L. P. W., and Zoghbi, H. Y. (1993) Expansion of an unstable trinucleotide CAG repeat in spinocerebellar ataxia type 1. Nat Genet 4, 221226.

(21) Kawaguchi, Y., Okamoto, T., Taniwaki, M., Aizawa, M., Inoue, M., Katayama, S., Kawakami, H., Nakamura, S., Nishimura, M., Akiguchi, I., Kimura, J., Narumiya, S., and Kakizuka, A. (1994) CAG expansions in a novel gene for Machado-Joseph disease at chromosome 14q32.1. Nat Genet 8, 221-228.

(22) Moriarty, A., Cook, A., Hunt, H., Adams, M. E., Cipolotti, L., and Giunti, P. (2016) A longitudinal investigation into cognition and disease progression in spinocerebellar ataxia types 1, 2, 3, 6, and 7. Orphanet $J$ Rare Dis 11.

(23) Bauer, P., Kraus, J., Matoska, V., Brouckova, M., Zumrova, A., and Goetz, P. (2004) Large de novo expansion of CAG repeats in patient with sporadic spinocerebellar ataxia type 7. J Neurol 251.

(24) Nakamura, K. (2001) SCA17, a novel autosomal dominant cerebellar ataxia caused by an expanded polyglutamine in TATA-binding protein. Hum Molec Genet 10, 14411448.

(25) Zhuchenko, O., Bailey, J., Bonnen, P., Ashizawa, T., Stockton, D. W., Amos, C., Dobyns, W. B., Subramony, S. H., and Zoghbi, H. Y. (1997) Autosomal dominant cerebellar ataxia (SCA6) associated with small polyglutamine expansions in the a $1 \mathrm{~A}$ voltage-dependent calcium channel Nat Genet 15, 62-9.

(26) Choubtum, L., Witoonpanich, P., Hanchaiphiboolkul, S., Bhidayasiri, R., Jitkritsadakul, O., Pongpakdee, S., Wetchaphanphesat, S., Boonkongchuen, P., and Pulkes, T. (2015) Analysis of SCA8, SCA10, SCA12, SCA17 and SCA19 in patients with unknown spinocerebellar ataxia: a Thai multicentre study. BMC Neurol 15.

(27) Katsuno, M., Adachi, H., Doyu, M., Minamiyama, M., Sang, C., Kobayashi, Y., Inukai, A., and Sobue, G. (2003) Leuprorelin rescues polyglutamine-dependent phenotypes in a transgenic mouse model of spinal and bulbar muscular atrophy. Nat Med 9, 768-773.

(28) Tobin, A. J., and Signer, E. R. (2000) Huntington's disease: the challenge for cell biologists. Trends in Cell Biology 10, 531-536.

(29) Snell, R. G., MacMillan, J. C., Cheadle, J. P., Fenton, I., Lazarou, L. P., Davies, P., MacDonald, M. E., Gusella, J. F., Harper, P. S., and Shaw, D. J. (1993) Relationship between trinucleotide repeat expansion and phenotypic variation in Huntington's disease. Nat Genet 4, 393-397. 
(30) Andrew, S. E., Paul Goldberg, Y., Kremer, B., Telenius, H., Theilmann, J., Adam, S., Starr, E., Squitieri, F., Lin, B., Kalchman, M. A., Graham, R. K., and Hayden, M. R. (1993) The relationship between trinucleotide (CAG) repeat length and clinical features of Huntington's disease. Nat Genet 4, 398-403.

(31) Ross, C. A., Poirier, M. A., Wanker, E. E., and Amzel, M. (2003) Polyglutamine fibrillogenesis: The pathway unfolds. Proc Natl Acad Sci USA 100, 1-3.

(32) Wetzel, R. (2012) Physical chemistry of polyglutamine: Intriguing tales of a monotonous sequence. $J$ Mol Biol 421, 466-490.

(33) Legleiter, J., Mitchell, E., Lotz, G. P., Sapp, E., Ng, C., DiFiglia, M., Thompson, L. M., and Muchowski, P. J. (2010) Mutant Huntingtin Fragments Form Oligomers in a Polyglutamine Length-dependent Manner in Vitro and in Vivo. J Biol Chem 285, 1477714790.

(34) Lajoie, P., and Snapp, E. L. (2010) Formation and Toxicity of Soluble Polyglutamine Oligomers in Living Cells. PLoS ONE (Chirico, G., Ed.) 5, e15245. (35) Nucifora, L. G., Burke, K. A., Feng, X., Arbez, N., Zhu, S., Miller, J., Yang, G., Ratovitski, T., Delannoy, M., Muchowski, P. J., Finkbeiner, S., Legleiter, J., Ross, C. A., and Poirier, M. A. (2012) Identification of Novel Potentially Toxic Oligomers Formed in Vitro from Mammalian-derived Expanded huntingtin Exon-1 Protein. J Biol Chem 287, 16017-16028.

(36) Kim, Y. E., Hosp, F., Frottin, F., Ge, H., Mann, M., Hayer-Hartl, M., and Hartl, F. U. (2016) Soluble Oligomers of PolyQ-Expanded Huntingtin Target a Multiplicity of Key Cellular Factors. Molec Cell 63, 951-964.

(37) Drombosky, K. W., Rode, S., Kodali, R., Jacob, T. C., Palladino, M. J., and Wetzel, R. (2018) Mutational analysis implicates the amyloid fibril as the toxic entity in Huntington's disease. Neurobiol Dis 120, 126-138.

(38) Pieri, L., Madiona, K., Bousset, L., and Melki, R. (2012) Fibrillar a-Synuclein and Huntingtin Exon 1 Assemblies Are Toxic to the Cells. Biophys J 102, 2894-2905.

\section{A8. Brief Overview of Additional Information for Instructors}

The material following this manuscript is a collection of the key components of the Supporting Information document. Grading rubrics, questionnaires, student manuals, equipment lists, CAS registries and protocols were not included for conciseness. Sections included in the lab summary and instructor notes contain important details to aid in the execution of the new lab, including adaptations and troubleshooting tips collected in several semesters of teaching this new laboratory exercise here at West Virginia University for biochemistry undergraduate students.

\section{A8.1 Lab Summary for Instructors}


Huntington disease (HD) is a fatal neurodegenerative disorder characterized by psychiatric symptoms, cognitive degeneration and motor instabilities. ${ }^{1}$ A mutation in the huntingtin gene caused by a CAG trinucleotide repeat results in a polyglutamine (polyQ) expansion in the translated huntingtin protein (htt). This polyQ expansion spurs htt aggregation into fibrils and ultimately into intracellular proteinaceous inclusions. ${ }^{2,3}$ Disease onset is directly correlated with polyQ length where 35-39 repeats may result in disease, 40-60 repeats result in adult onset, and repeat lengths greater than 60 are observed in juvenile disease onset. ${ }^{2}$ Further, there is increasing evidence that htt aggregation may be associated with disease progression and severity, suggesting a larger need for research exploring aggregate formation. ${ }^{2,3}$

Novel strategies that directly target the HD gene and prevent production of the toxic mutant htt are being investigated. One current strategy involves an antisense oligonucleotides (ASO)-based RNase activation system via spinal injection to HD patients. ${ }^{4}$ While successful, HD progresses slowly, and this treatment becomes expensive if prolonged. Additionally, protein misfolding and abnormal htt can interfere with several cellular processes, including cell trafficking. ${ }^{1,2}$ This presents several challenges not only for obtaining cost-effective treatments but in understanding the complete mechanism of the disease progression. ${ }^{5}$ Investigation into physiologically relevant or less invasive therapeutic drug targets exploring htt folding remains a necessary and interesting topic of investigation.

As an undergraduate student, the independent use of advanced instrumentation is not always a possibility due to budgetary limitations and the restrictions caused by a scheduled laboratory period, which greatly limits individualistic exploration and critical 
thinking. These limitations can be modified if the student participates in undergraduate research; however, this is not always a possibility for all students. Thus, we have developed a laboratory experiment where it is now possible to expand protein growth and purification methods to additional proteins that have otherwise been underutilized in an undergraduate biochemical setting through modifications made in the laboratory timeline. Specifically, students will complete experimental preparative methods (E. Coli growth, protein purification, etc.) that mirror advanced protocols completed in biochemical research through guided-inquiry and then complete independent experimentation with the htt protein quantifying amyloid formation and prevention using macromolecular crowders. These technical experiences are relevant in a broader, real world context. For example, high throughput assays in plate format are a common method of screening for potential therapeutics, which relies on many of the design principles and data analysis employed in this laboratory experiment.

\section{Time and curriculum level}

Students should perform the experiment in an advanced laboratory course after they have been introduced to basic biochemistry techniques and lab practices; skills and topics relating to protein expression and purification, kinetics, instrumentation, and critical thinking will be utilized. The laboratory experiment combines aspects of biochemistry and biophysical chemistry, and therefore can be utilized for several topics of discussion in biochemistry or physical chemistry courses. Completion of this laboratory experiment should occur during lectures that include topics of protein expression, protein structure/folding, spectroscopic analytical procedures, and kinetics. In total, this experiment requires six, three-hour laboratory sessions. Students work in 
larger groups during experimentation for four, three-hour sessions, and two, three-hour laboratory sessions for oral and written scientific presentations with emphasis on developing advanced written and oral communication skills required for upper-level education and STEM careers.

Procedures, techniques, and concepts presented

During this experiment, students will utilize advanced laboratory techniques to determine the effects of macromolecular crowders on htt aggregation kinetics. After preparing solutions, such as lysogeny broth (LB) and buffers, students express httexon1 containing an aggregation prone polyQ domain as a GST-fusion protein in Escherichia Coli (E. coli). Once optimum density of E. coli is confirmed in the large batch inoculations through optical density measurements, protein expression is induced via isopropyl- $\beta$-D-thiogalactoside (IPTG). The cells are then washed and harvested as pellets via centrifugation. For purification, these pellets are re-suspended in buffer and lysed by treatment with lysozyme followed by probe sonication. Cellular debris is pelleted and discarded. The cell lysate, which contains the fusion protein, is diluted with buffer and loaded onto a prepared glutathione-S-transferase (GST)-based affinity spin column for purification via centrifugation. Upon completion, protein concentration is preliminarily determined by a Bradford assay, using a standard curve generated with bovine serum albumin (BSA) standards. The purified protein is dialyzed for 48 hours at $4^{\circ} \mathrm{C}$, with two dialysis buffer exchanges. The concentration of the dialyzed protein is determined using the Bradford assay; however, the purified protein usually needs to be concentrated via centrifugal concentrators prior to use in aggregation assays. 
Students will also prepare thioflavin T (ThT) stocks and several macromolecular crowder solutions for testing; they will determine the amount of protein per well that is needed for analysis in addition to calculating the appropriate concentrations of thioflavin T and crowders. Students then plan and prepare their samples in triplicate using a 96well plate and complete kinetic testing via ThT fluorescence using a microplate reader. Once completed, students analyze and present their finding to their peers.

Performing this laboratory experiment provides students with an opportunity to utilize important upper level laboratory techniques such as protein expression and purification, affinity-based column chromatography, and fluorescence-based assays. These experiments enable the student to conceptualize how a protein such as $\mathrm{htt}$ functions and is analyzed. Students also recognize how simple spectroscopic techniques can be efficiently used to monitor the progress of purification and determine an unknown concentration of a protein. Students can compare htt aggregation in the presence of macromolecular crowders, providing first-hand experimental knowledge of the potential impact of the intracellular crowded environment on htt. This laboratory lies at the interface between biology and physical chemistry, an important interdisciplinary field to which many students are not exposed, particularly in a laboratory setting. Additionally, students become familiar with useful data analysis and presentation techniques that will be advantageous in advanced STEM educational programs and careers.

\section{Usefulness of experiment}

This laboratory will be useful for illustrating principles of biochemistry and biophysical chemistry, including protein expression, purification, and aggregation. 
Protein purification and aggregation is monitored through analytical techniques, including spectroscopic and fluorescence-based assays. The hands-on experience that this laboratory experiment provides conceptualization of how these simple techniques can be applied to understanding relevant, biochemical phenomena, e.g. toxic protein aggregation. Additionally, students gain experience using a microplate reader to kinetically monitor aggregation and investigate how molecularly crowded environments that mimic the cytosol can influence this process. Further, using a disease-relevant protein such as htt provides real world relevance and a greater appreciation of amyloid diseases. Students gain a greater understanding of the complete, fundamental process of protein expression and purification followed by application of the obtained protein in an assay that mimics exploratory laboratory research that one would encounter in industry or in academic research labs.

\section{Summary of student evaluations}

Student evaluations were completed following each week of experimentation with 50 student participants spanning two semesters. From the results of the evaluations, most students did not have extensive experience working in a research laboratory nor did most complete anything like the weekly experimentation in previous coursework or research experiences.

Common responses to both protein expression (Week \#1) and protein purification (Week \#2) experimentations included "the experiment included components never done before in other graded labs like stock preparation" and "this lab was good to learn about lab prep work and what actually goes into prepping for experiments as a whole". Throughout the protein purification process (Week \# 2), students valued the use of 
"multiple higher-level instruments" and techniques for completing the experiment and "learning multiple things in a lab period that were touched upon in lecture or in the past coursework". Students also reported enjoying group work, which "allowed open communication and collaboration" as the experiment was being completed. One comment indicated that it was interesting to learn a biochemical approach to column chromatography when students are usually only exposed to a version of it in organic chemistry.

The Excel workshop (Week \#3) reported an average of $76 \%$ of students who valued the learning experience, and an average of $95 \%$ of students suggested it should be continued in future semesters. Students enjoyed the "scientific approach to excel", including the ways to "create data plots that resemble those seen in literature" and appreciated tips on how to simplify data analysis.

Week \#4 of experimentation, which consisted of independent experimental design and implementation, showed students overall enjoying the increased hands-on working opportunities; students also valued the heighten anticipation of completing their designed experiment and seeing how the individual weekly laboratories were applied to that final design. Student data indicated an average of $82 \%$ of students enjoying the sixweek long experiment overall as well as an average of approximately $80 \%$ of students enjoying each week individually.

\section{A8.2 Instructor Notes}

Pre-laboratory assignment 
It is recommended that students complete Experiment \# 0 prior to the first threehour laboratory session. Completing this experiment is optional; however, it is suggested for students to practice making biological solutions that are not conventionally generated in a typical undergraduate laboratory course. Further, completing this experiment will reduce laboratory time, ensuring completion of the htt laboratory experiment, and provide students with an obvious example of how preparation is essential in biochemical research.

\section{Alternative experiments}

This laboratory experiment is advantageous in that it can be readily adapted to investigate other relevant intracellular components. For example, using the ThT assay, students could explore the use of other physiologically relevant macromolecular crowder molecules or small molecules, such as epigallocatechin gallate (EGCG), curcumin and rizuole. Potential therapeutic drug screenings could also be completed where students could compare and differentiate their findings on different drug targets. Another simplistic laboratory assay, such as polydiacetylene assay (PDA), could be used to study the effects of crowders, small molecules or potential drugs on htt binding to lipids. This assay may possibly be expanded further by exploring different lipid systems or incorporating lipids into the ThT assay. Depending on instrumentation and skill level of students, variation of instrumentation in the final experiment can occur. Circular dichroism (CD), atomic force microscopy (AFM), solid state nuclear magnetic resonance (sSNMR), and scanning electron microscope (SEM) are all examples that can be used to modify the experiment. For example, AFM can be used to study the morphologies of various aggregates produced and can complement kinetic assays 
using similar experimental conditions. Other techniques including immunohistology experiments (Western blots, slot and dot blots) can also be used to identify different protein aggregates.

\section{Laboratory preparation}

Students can work in large groups with individual students taking turns completing tasks at various stages throughout each week of experimentation. Students should be provided with appropriate equipment and chemicals.

\section{Solution preparation}

Solution preparation and some procedural steps will need to be completed outside of the scheduled laboratory period by the instructor or teaching assistant. Several salt, bleach and buffer solutions will need to be prepared as described. During Week \#1, E. coli. large batch inoculations from the overnight culture should be started and close to the appropriate optical density (OD) prior to the start of the first laboratory session. Depending on the polyQ repeat length used, the incubation period will need to be started 24 hours in advance with the expression step occurring between two and four hours prior to the start of the lab session. In Week \#2, the protein should be dialyzed for 48 hours with aliquots of dialysis buffer being replaced twice. Note: The protein prepared by students will be useable for up to 10 days, however, ideal use would be within 5-7 days.

Multiple htt samples can be combined to generate mock data for use in Week \#3 experimentation. Additionally, a large quantity of protein should be purified three days prior to Week \#4 laboratory session to ensure freshness and prevent seeding. If 
preferred, a larger sample of protein could be purified using a liquid chromatography (LC) system or multiple purifications could be completed and combined using the spin column purification method as described in Week \#2.

\section{Instrumental instructions}

Prior to starting each three-hour laboratory session, instructors should turn on the refrigerated centrifuge and plate reader one to two hours prior to the experiment to ensure appropriate temperature equilibration. UV-Vis spectrophotometer, sonicator, and bath sonicator can all be turned on during the experiment as they do not have lengthy start up times. Additional details are outlined in the experimental procedures each week.

\section{Tips for success}

Notes are provided to students to assist in proper experimental completion; however, some additional areas where difficulties may occur should be announced to students. Starting with the first week, encourage students to think of how the instrumentation and weekly experiment applies to their overall experimental objectives. Participation by each student should be monitored during the laboratory sessions, as students may not be self-motivated, interested in teamwork, or have no experience in gradate-related research labs. Encouragement and deeper conceptual inquiries with the instructor are encouraged, as these interactions are more representative of a research setting and helps to promote the desired self-guided approach; opportunities to discuss the written laboratory reports and oral presentations prior to submission and in-class presentations are also suggested. 


\section{Troubleshooting}

Special care should be taken with some experimental protocols. For example, the probe sonicator has a very delicate tip that is easily damaged. Students should be encouraged to handle the probe sonicator gently and monitor the protein consistency for any necessary adjustments in probe wattage. Additionally, if the concentration results in a lower yield than expected, procedural modifications can be made by reducing the number of wells to two instead of three or reducing the protein concentration per well. This ensures useful data will be achieved and demonstrates the unpredictability of scientific research.

\section{Adapting This Experiment}

$\mathrm{Htt}$ is useful at demonstrating additional biochemistry topics, such as protein structure and aggregation mechanisms, depending on instrumentation and desired difficulty level. If htt is not available, the protein could easily be substituted with $\beta$ amyloid or $\alpha$-synuclein peptides. These peptides can be ordered commercially but would not require purification, which would eliminate cell growth and purification from the experimental procedure. However, this change could allow for the introduction of other experimental methods and instrumentation involved in peptide preparation, including lyophilizers and vacuum pumps.

Students could prepare the stock solutions required for the experiment, including $1 \mathrm{M} \mathrm{Na}_{2} \mathrm{PO}_{4}, 1 \mathrm{M} \mathrm{NaH}_{2} \mathrm{PO}_{4}, 5 \mathrm{M} \mathrm{NaCl}, 1 \mathrm{M}$ Tris $(\mathrm{pH} 7)$ and a $10 \%$ bleach solution; this would accompany in-lab preparation of purification solutions such as dialysis buffer, buffer A, buffer B and aggregation buffer. 
Students should be cognizant of experimental procedures completed outside of the scheduled laboratory period, such as dialysis or expression steps. If available, students can purify larger quantities of the protein using a low-pressure liquid chromatography (LC) system, which would provide protein for multiple experimental comparisons on one microfluorescent plate. Comparisons of similar or different targets could be completed with students generating a scientific case for why their target should be explored further.

Calculations could be removed or added to modify the difficulty level during each week of experimentation. Additionally, a more in-depth literature search of htt along with a chosen assay could be conducted.

\section{A8.3 References}

(1) Truant, R., Atwal, R., and Burtnik, A. (2006) Hypothesis: huntingtin may function in membrane association and vesicular trafficking. Biochem Cell Biol 84, 912-917.

(2) Velier, J., Kim, M., Schwarz, C., Kim, T. W., Sapp, E., Chase, K., Aronin, N., and DiFiglia, M. (1998) Wild-Type and Mutant Huntingtins Function in Vesicle Trafficking in the Secretory and Endocytic Pathways. Exp Neurol 152, 34-40. 